\title{
INDISPENSABILITY ARGUMENTS IN THE PHILOSOPHY OF MATHEMATICS
}

\author{
By \\ Mark Colyvan
}

A thesis submitted for the degree of

Doctor of Philosophy of

The Australian National University.

October 1997 
Indispensability Arguments in the Philosophy of Mathematics

Copyright (c) 1997 by Mark Colyvan

Philosophy Program

Research School of Social Sciences

Institute of Advanced Studies

The Australian National University

Canberra, ACT, 0200

Australia

Page $\mathrm{v}$ constitutes an extension of the copyright page.

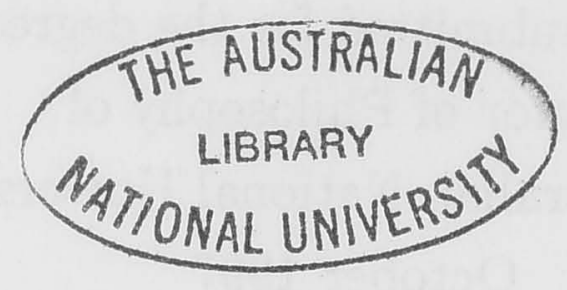

This document was prepared using $\mathrm{HT}_{\mathrm{E}} \mathrm{X} 2 \varepsilon$. 


\section{DECLARATION}

I certify that the substance of this thesis has not already been submitted for any degree and is not currently being submitted for any other degree.

I certify that to the best of my knowledge any help received in preparing this thesis, and all sources used, have been acknowledged. 



\section{Preface}

This thesis draws upon material which has been published as papers in various journals, so I'd like to thank those publishers for permission to use that material again here. The copyright for previously published material remains with the relevant journals.

Much of chapter 4 (and some of chapter 1) comes from my paper 'Confirmation Theory and Indispensability' [Col 9?] forthcoming in Philosophical Studies. Chapter 5 consists almost entirely of my paper 'In Defence of Indispensability' [Col 98a] forthcoming in Philosophia Mathematica. Chapter 7 draws heavily on my paper 'Is Platonism a Bad Bet?' [Col 98b] forthcoming in The Australasian Journal of Philosophy and my 'Review of Morton, A. and Stich, S. (eds.) Benacerraf and his Critics' [Col 97] in The Australasian Journal of Philosophy, Vol. 75, No. 2, (June 1997).

As for more personal acknowledgements; I'd like to thank Frank Jackson, Jack Smart, Peter Roeper, Graham Oppy and Peter Menzies, all of whom have been members of my advisory panel at some time or other. Their interest, guidance and advice has had a significant effect on both my own intellectual development and on this thesis.

In 1995, during my second year of candidature, I was fortunate enough to visit Italy to attend two conference (the 10th ICLMPS conference in Florence and a conference in Mussomeli Sicily on 'Truth in Mathematics'), both of which were extremely beneficial in the preparation of this thesis. I also took the opportunity to visit the UK and the USA to speak with many philosophers, all of whom I am extremely grateful to for their time and hospitality. Those I visited and to whom I owe significant intellectual debts include Michael Potter (Cambridge), Alex Oliver (Cambridge), Hartry Field (City University of New York), Paul Benacerraf (Princeton), Gideon Rosen (Princeton), W.V. Quine (Harvard), Michael Resnik (University of North Carolina, Chapel Hill) and Penelope Maddy (University of 
California, Irvine). I have also enjoyed ongoing correspondence with a number of these people.

The first semester of 1996 I spent at the University of New England where I had many fruitful conversations with Drew Khlentzos and Peter Forrest. I am grateful to them both for their time and interest in my work.

I consider myself fortunate to have been accepted as a graduate student in the Philosophy Program of the Research School of Social Sciences at the Australian National University. For a variety of reasons this is a very good place to do one's graduate study. Not least of these reasons is the camaraderie amongst graduate students, so I'd like to thank all my fellow graduate students in the philosophy program at RSSS during my time there. In particular, David Ryan, Philip Gerrans and James Chase, with whom I've shared offices at various times and with whom I've spent many hours discussing a variety of philosophical issues. I'm further indebted to James Chase for his meticulous proof-reading and for his very helpful comments on an earlier draft of this thesis.

Another commendable feature of the RSSS is the number of congenial visiting philosophers that spend time here. A few, in particular, spring to mind as deserving mention for their impact on my work. They are Keith Lehrer from the University of Arizona, Bernard Linsky from the University of Alberta and Alan Musgrave from the University of Otago. I have also profited from conversations and/or correspondence with Jody Azzouni (Tufts), Alan Baker (Princeton), John Bigelow (Monash), Brian Birchall (UNE), Colin Cheyne (Otago), Ian Gold (ANU), Geoffrey Hellman (Minnesota), Bob Meyer (ANU), Philip Pettit (ANU) and Ed Zalta (Stanford).

Finally, I'd like to thank my partner Helen Regan for her constant love, companionship and proof-reading over the three-and-a-bit years it's taken to complete this thesis.

Moving on to practical matters; I employ a fairly standard scientific referencing system in this thesis. This system uses tags to refer to works in the bibliography. For instance, the tag '[Qui 60]' refers to:

Quine, W.V. Word and Object. Massachusetts Institute of Technology

Press and John Wiley and Sons, New York, 1960.

To find the full publication details, one needs to look up the tag in the bibliographythese are ordered alphabetically, then numerically - and read off the corresponding bibliographic entry. The tag, however, gives some bibliographic information: it provides the first three (or in some cases four) letters of the (first) author's name 
and the date of publication of the work in question. When the name of the author and/or the work are important to the discussion, these will be found in full in the text (along with a bibliographic tag of course).

When referring to some specific page(s) in a given work, the page(s) will be indicated inside the square bracket, separated from the bibliographic details by a comma. For example, [Qui 60, 248-251] refers to pages 248-251 in the abovementioned work by Quine. Sometimes it is necessary to mention a list of works; they will be listed inside the square brackets thus: [Qui 48, Qui 51, Qui 60] rather than individually. One last point concerning referencing; sometimes I will use a tag such as '[Qui 60]' as a noun (as in 'Quine showed in [Qui 60, 248-251] how to dispense with idealisations in physics') and sometimes as a bracketed, more conventional citation (as in 'Quine showed how to dispense with idealisations in physics [Qui 60, 248-251]').

I also borrow another referencing device from scientific writing: whenever I introduce a major definition, argument, principle or theorem, I present it in a distinctive environment which is numbered for ease of future reference. Whenever the reference is fairly remote from the definition/argument/principle/theorem in question, I will also provide the relevant page number.

Lastly, I need to say a little about a stylistic matter. I often speak of certain entities being dispensable or indispensable to a given theory. Strictly speaking it's not the entities themselves that are dispensable or indispensable, but rather it's the postulation of or reference to the entities in question that may be so described. Having said this, though, I will, for the most part, talk about entities being dispensable or indispensable, eliminable or non-eliminable and occurring or not occurring. I do this for stylistic reasons but I apologise in advance to any reader who is irritated by this. 



\section{Abstract}

In this thesis I look at indispensability arguments in the philosophy of mathematics. Such arguments typically urge us to place mathematical entities on the same ontological footing as other theoretical entities indispensable to our best scientific theories. These arguments, however, have come under serious scrutiny in recent times with many influential philosophers unconvinced of their cogency. Although I will discuss the various forms indispensability arguments take, I will largely be concerned with defending the Quine/Putnam version of the argument against recent attacks from Hartry Field, Penelope Maddy, Elliott Sober and others.

In order to appreciate the full force of the Quine/Putnam indispensability argument it is necessary to consider the Quinean backdrop from which it emerges. This backdrop consists of the doctrines of holism and naturalism. The latter in particular is crucial to the whole indispensability debate, so I spend a considerable portion of this thesis discussing naturalism and its relevance to indispensability theory. 



\section{Contents}

Preface $\quad$ V

Abstract ix

1 Mathematics and its Applications $\quad 1$

1.1 Realism and Anti-realism in Mathematics . . . . . . . . . . . 2

1.2 Indispensability Arguments . . . . . . . . . . . . . 6

1.2.1 Introducing Indispensability Arguments . . . . . . . . . . . 6

1.2.2 The Quine/Putnam Indispensability Argument . . . . . . . . 10

1.2 .3 Other Indispensability Arguments . . . . . . . . . . . . 13

1.3 The Road Ahead . . . . . . . . . . . . . . . . . . . . 18

2 The Quinean Backdrop 21

2.1 Introducing Naturalism . . . . . . . . . . . . . . . . . 22

2.2 Quinean Naturalism . . . . . . . . . . . . . . . . . . . . 23

2.3 The Methodologies of Philosophy and Science . . . . . . . . . . 26

2.3.1 The 'Two Dogmas' Argument . . . . . . . . . . . . . . 27

2.3.2 How Useful is the Distinction? . . . . . . . . . . . . . 29

2.4 The Causal Version of Naturalism . . . . . . . . . . . . . . . 32

2.5 Holism . . . . . . . . . . . . . . . . . . . . . . . . . 34

2.6 The First Premise Revisited . . . . . . . . . . . . . . . 37

3 The Eleatic Principle $\quad 39$

3.1 The Inductive Argument . . . . . . . . . . . . . . . . . . . . . 41

3.2 The Epistemic Argument . . . . . . . . . . . . . . . . . . . . . 43

3.3 The Argument from Causal Explanation . . . . . . . . . . . . . . 46

3.3 .1 The Bending of Light . . . . . . . . . . . . . . . . 48

3.3 .2 Stellar Stability . . . . . . . . . . . . . . . . . . . . 49 
3.3.3 Antipodal Weather Patterns . . . . . . . . . . . . . 51

3.3.4 The FitzGerald-Lorentz Contraction . . . . . . . . . . 52

3.4 Causal Relevance . . . . . . . . . . . . . . . . . 53

3.5 Rejection of Inference to the Best Explanation . . . . . . . . . 56

3.5.1 Ellis's Argument . . . . . . . . . . . . . . . . . 56

3.5 .2 Cartwright's Argument . . . . . . . . . . . 58

3.6 The Moral . . . . . . . . . . . . . . . . . . . . . . . . . . . . . . . . . . 60

3.7 Recapitulation . . . . . . . . . . . . . . . . 63

4 Field's Fictionalism $\quad \mathbf{6 5}$

4.1 The Science Without Numbers Project . . . . . . . . . . . . 67

4.1 .1 Conservativeness . . . . . . . . . . . . . 68

4.1 .2 Nominalisation . . . . . . . . . . . . . 70

4.2 What is it to be Indispensable? . . . . . . . . . . . 74

4.3 The Role of Confirmation Theory . . . . . . . . . . . . . 76

4.4 The Role of Mathematics in Physical Theories . . . . . . . . . . 79

4.4.1 The Complex Numbers ... . . . . . . . . . . 79

4.4 .2 Maxwell's Equations . . . . . . . . . . . . . . . 81

4.4 .3 The Dirac Equation . . . . . . . . . . . . . . . 82

4.4.4 The Lorentz Transformations . . . . . . . . . . . . . . 83

4.5 Review of Field's Fictionalism . . . . . . . . . . . . . 86

5 Maddy's Objections $\quad 91$

5.1 The Objections . . . . . . . . . . . . . . . . . . 92

5.1 .1 The Scientific Fictions Objection . . . . . . . . . . . . . 92

5.1 .2 The Role of Mathematics in Science . . . . . . . . . . . 93

5.1.3 The Mathematical Practice Objection . . . . . . . . . . . . 93

5.2 Maddy's Naturalism . . . . . . . . . . . . . . . . . . 96

5.3 Defending the Indispensability Argument . . . . . . . . . . . . 99

5.3.1 The Scientific Fictions Objection Revisited . . . . . . . . 99

5.3.2 The Role of Mathematics in Science Revisited . . . . . . . . . 102

5.3.3 The Mathematical Practice Objection Revisited . . . . . . . 105

5.4 Review of Maddy's Objections . . . . . . . . . . . . . 112

6 Further Objections 115

6.1 The Obviousness of Some Mathematical Truth . . . . . . . . . . 116

6.2 The Unfalsifiability of Mathematical Claims . . . . . . . . . . . . 123 
6.3 The Sober Objection . . . . . . . . . . . . . . . . 127

6.3 .1 Contrastive Empiricism . . . . . . . . . . . . 127

6.3 .2 The Objection . . . . . . . . . . . . . . . . . 129

6.3 .3 A Residual Worry . . . . . . . . . . . . . . . . 134

6.4 Is Mathematics Contingent? . . . . . . . . . . . . . . . 137

6.4.1 What is the Contingency Contingent on? . . . . . . . 138

6.4 .2 The Argument from Insularity . . . . . . . . . . . . . 140

7 Conclusion $\quad 143$

7.1 What the Indispensability Argument Doesn't Say . . . . . . . . . . . 144

7.2 The Benacerraf Challenges . . . . . . . . . . . . . . . . 149

7.2.1 What Numbers Could Not Be . . . . . . . . . . . 150

7.2.2 Naturalising Mathematical Epistemology . . . . . . . . . . 153

7.3 A Slippery Slope?. . . . . . . . . . . . . . . . . . 157

$\begin{array}{lr}\text { A Summary } & 159\end{array}$

$\begin{array}{lr}\text { B Notation } & 163\end{array}$

$\begin{array}{lr}\text { Bibliography } & 165\end{array}$

$\begin{array}{lr}\text { Index } & 181\end{array}$ 



\section{Chapter 1}

\section{Mathematics and its Applications}

For some time now ontological debates in the philosophy of mathematics have been dominated by three arguments. The first of these is the Benacerraf indeterminacy objection to the natural numbers being identified with sets. ${ }^{1}$ The second argument, also due to Benacerraf, is the well known epistemological problem for Platonism. That is, if mathematical objects such as functions, numbers and sets have mind independent, though admittedly abstract, existence, how is it that we have knowledge of them $?^{2}$ (This is often referred to as the problem of naturalising mathematical epistemology.) The third argument is the Quinean argument that mathematical entities are indispensable to our best physical theories and therefore share the ontological status of scientific entities. The first two of these arguments are taken to raise problems for the position called Platonism or mathematical realism, while the third is usually taken to be an argument for Platonism; perhaps, as Hartry Field suggests, "the only non-question-begging" argument for Platonism [Fie 80, 4].

Although I will return to the two Benacerraf problems in the final chapter of this thesis, for the most part I'll be concerned with the indispensability argument. In particular, I will be defending this argument against some of its recent critics. Before discussing the indispensability argument in more detail, though, some preliminaries are in order. Since the indispensability argument is supposed to have

\footnotetext{
${ }^{1}$ For example, should we identify the natural number three with von Neumann's $\{\emptyset,\{\emptyset\},\{\emptyset,\{\emptyset\}\}\}$ or with Zermelo's $\{\{\{\emptyset\}\}\}$ ? See [Ben 65] for further details.

${ }^{2}$ Although Benacerraf made explicit reference to the causal theory of knowledge in his formulation of the argument [Ben 73], the argument can be reconstructed so that no such reference is required (as in [Fie 89, 25-26]).
} 
serious implications for the debate about realism and anti-realism in mathematics, I need to say a little about what I take realism and anti-realism to be, and also what I take this debate to be about in the case of mathematics. I deal with this in the following section. Then, in section 1.2, I give an introduction to indispensability arguments with particular attention to the Quinean indispensability argument. In section 1.3 I give an outline of the chapters ahead and how they bear on the central task of this thesis: the defence of the Quinean indispensability argument.

\subsection{Realism and Anti-realism in Mathematics}

There are many different ways to characterise realism and anti-realism in mathematics. Perhaps the most common way is as a thesis about the existence or non-existence of mathematical entities. Thus, according to this conception of realism, mathematical entities such as functions, numbers and sets have mind and language independent existence or, as it is also commonly expressed, we discover rather than invent mathematical theories (which are taken to be a body of facts about the relevant mathematical objects). This is usually called metaphysical realism. Anti-realism, then, is the position that mathematical entities do not enjoy mind independent existence or, alternatively, we invent rather than discover mathematical theories. According to this characterisation, a realist believes that Fermat's Last Theorem ${ }^{3}$ was true before Wiles's proof and, indeed, even before Fermat first thought of his now famous theorem. This is because, according to the realist, the integers exist independently of our knowledge of them and Fermat's conjecture, it turns out, is a fact about them.

Another way realism is often characterised is via truth. For instance Michael Dummett says that realism is "the belief that statements of the disputed class possess an objective truth-value, independently of our means of knowing it: they are true or false in virtue of a reality existing independently of us" [Dum 78a, 146]. ${ }^{4}$ This is usually called semantic realism. Hilary Putnam is another who prefers this way of characterising realism and points out that according to this view it is possible to be a mathematical realist without being committed to mathematical objects - realism is

\footnotetext{
${ }^{3}$ This theorem states that there are only trivial integer solutions of the equation $x^{n}+y^{n}=z^{n}$ when $n>2$. It is called 'Fermat's Last Theorem' because Fermat noted in the margin of a book that he had a proof of this but that the margin was too small to contain it. Fermat never produced a proof and, what is more, it seems exceptionally unlikely that he had a proof, given the difficulty of the proof first produced by Andrew Wiles in 1993. See [Sin 97] for further details.

${ }^{4}$ You would probably also require that some of the statements in the disputed class be true, for otherwise fictionalists about some discourse turn out to be realists!
} 
about objectivity, not objects [Put 79a, 69-70]. For example, suppose mathematical discourse is reinterpreted so that it does not refer to mathematical objects and this reinterpreted discourse is true or false independently of our knowledge of it. Such a position satisfies Dummett's characterisation of realism, and yet there are no entities peculiar to mathematics. Indeed Putnam's modal account of mathematics is a nice example of a realist view of mathematics that has no mathematical objects. ${ }^{5}$

Putnam's claim that it is the objectivity of mathematics that is really the issue is surely correct for a great many contexts, but not when one is doing metaphysics! Certainly one reason many philosophers and mathematicians find the view that mathematical objects exist appealing is that it does provide mathematics with the desired objectivity. For instance, as Penelope Maddy points out [Mad 96b, 68-69], Kurt Gödel was drawn to mathematical realism for this very reason. Platonism purported to provide the independent questions of mathematics with objective answers. ${ }^{6}$ If this were all there was at issue, then Putnam's account of realism would suffice, but objectivity is not the only issue. The important question in the ontology of mathematics is: Do mathematical objects exist? I take this question to be interesting and worth pursuing. The Dummett/Putnam characterisation of realism and anti-realism, however, does not address this question. ${ }^{7}$

Since, in this thesis, my interests are metaphysical, I will be concerned with metaphysical realism, not semantic realism. I will, however, speak only of realism, by which I will mean metaphysical realism. I should stress that I am not presupposing that metaphysical realism is the correct account of realism-my use of 'realism' to mean 'metaphysical realism' is merely terminological. ${ }^{8}$ While on matters terminological, I should also point out that, in keeping with most of the modern literature in the area, I will use the terms 'mathematical realism' and 'Platonism' interchangeably.

So I take Platonism to be the view that mathematical objects exist and, what is more, that their existence is not mind or language dependent. I also take it that according to Platonism mathematical statements are true or false in virtue of the

\footnotetext{
${ }^{5}$ On this view, mathematical claims are reinterpreted as modal claims. For instance, the claim that numbers exist becomes the claim that $\omega$-sequences are (mathematically) possible. See [Put 67] for further details.

${ }^{6}$ Indeed, Maddy too has much sympathy with this style of motivation.

${ }^{7}$ This is not to say, however, that the Dummett/Putnam account is without merit. You might be inclined to think that what is important in realism is captured by Dummett's characterisation, but that questions concerning ontology are somewhat independent of issues of realism.

${ }^{8}$ The issue of explicating the best or correct account of realism is far too large to do justice to in this thesis. See [Wri 92] for an interesting discussion of this topic.
} 
properties of these mathematical objects. I do not mean to imply anything more than this. I do not, for instance, intend Platonism to mean that mathematical objects are causally inert, that they are not located in space-time or that they exist necessarily. Although such views are typically endorsed by Platonists, such endorsement is by no means universal. For example, Penelope Maddy [Mad 90] and John Bigelow [Big 88] have both put forward accounts of mathematics, which they describe as Platonist accounts, in which mathematical objects are located in spacetime and are part of the causal nexus. Others propose accounts where mathematical objects exist contingently. ${ }^{9}$ These accounts all postulate mathematical objects and, from an ontological point of view, this is what matters. I will thus use the term 'Platonism' to include them all.

Given the above characterisation of mathematical realism or Platonism we can reasonably ask about anti-realist accounts of mathematics. Clearly any account that denies that mathematical objects exist is anti-realist. I shall call such a position nominalism. There is, however, a second anti-realist position. This is the view that mathematical objects exist but that they are mind or language dependent. I agree with Hartry Field [Fie 89, 1-2] here that this last position is of little interest. The important question is whether mind and language independent mathematical objects exist or not. Having noted this second position I will now largely ignore it. I will thus speak of Platonism and nominalism as the two viable positions. ${ }^{10}$

There are various Platonist and nominalist strategies in the philosophy of mathematics, some of which we will meet in the course of this dissertation. Each of these has its own particular strengths and weaknesses. As I've already mentioned, Platonist accounts of mathematics generally have the problems of providing an adequate epistemology for mathematics and of explaining the apparent indeterminacy of number terms. On the other hand, nominalist accounts generally have trouble providing an adequate treatment of the wide and varied applications of mathematics in the empirical sciences. There is also the challenge for nominalism to provide a uniform semantics for mathematics and other discourse [Ben 73]. Although this challenge receives less attention than the other three arguments I mentioned earlier, I don't think that this lack of attention signifies that this challenge is of less importance. A believable nominalist account must provide an account of the semantics of mathematical discourse that is not ad hoc. It might be useful at this stage to

\footnotetext{
${ }^{9}$ As we shall see, I endorse such a view.

${ }^{10}$ You might consider my sense of nominalism here to include the second anti-realist position by thinking of nominalism as the view that no mind independent mathematical objects exist.
} 
outline a few of the different strategies encountered in the literature.

An important nominalist response to the above arguments is fictionalism. A fictionalist about mathematics believes that mathematical statements are, by and large, false. According to the fictionalist, mathematical statements are "true in the story of mathematics" but this does not amount to truth simpliciter. Fictionalists take their lead from some standard semantics for literary fiction. On many accounts of literary fiction 'Sherlock Holmes is a detective' is false (because there is no such person as Sherlock Holmes), but it is "true in the stories of Conan Doyle". The mathematical fictionalist takes sentences such as 'seven is prime' to be false (because there is no such entity as seven) but "true in the story of mathematics". The fictionalist thus provides a distinctive response to the challenge of providing a uniform semantics - all the usually accepted statements of mathematics are false. The problem of explaining the applicability of mathematics is more involved, and I will leave a discussion of this to chapter 4 , where I consider one particular version of fictionalism due to Hartry Field in more detail.

Many Platonist strategies in recent times have responded to the epistemological challenge by placing mathematical objects firmly in the physical realm. Thus Penelope Maddy in Realism in Mathematics [Mad 90] argued that we can see sets. When we see six eggs in a carton we are seeing the set of six eggs. This account provides mathematics with an epistemology consistent with other areas of knowledge by giving up one of the core doctrines of traditional Platonism - that mathematical entities are abstract. In response to the apparent indeterminacy of the reductions of other mathematical entities to sets, one popular Platonist strategy is to identify a given natural number with a certain position in any $\omega$-sequence. Thus, it doesn't matter that three can be represented as $\{\{\{\emptyset\}\}\}$ in Zermelo's $\omega$-sequence and $\{\emptyset,\{\emptyset\},\{\emptyset,\{\emptyset\}\}\}$ in von Neumann's $\omega$-sequence. What is important on this account is that the structural properties of the above two accounts are identical. This view is usually called 'structuralism' since it is the structures that are important, not the items that constitute the structures. ${ }^{11}$

These are not meant to be anything more than cursory sketches of some of the available positions. Some of these positions will arise again in the course of this dissertation, but for now I will be content with these sketches and move on to discuss indispensability arguments and how these arguments are supposed to deliver mathematical realism.

\footnotetext{
${ }^{11}$ See for example, [Res 81, Res 82, Res 97, Hel 89].
} 


\subsection{Indispensability Arguments}

\subsubsection{Introducing Indispensability Arguments}

One of the most intriguing features of mathematics is its applicability to empirical science. Every branch of science draws upon large and often diverse portions of mathematics, from the use of Lie groups in quantum mechanics to the use of differential geometry in cosmology. It's not only the physical sciences that avail themselves of the services of mathematics either. Biology, for instance, makes extensive use of difference equations and statistics. The roles mathematics plays in these theories is also varied. Not only does mathematics help with empirical predictions, it allows elegant and economical statement of many theories. Indeed, so important is the language of mathematics to many theories, that it is hard to imagine how some theories could even be stated without appeal to mathematics. Furthermore, looking at the world through mathematical eyes has, on more than one occasion, facilitated enormous breakthroughs in science. ${ }^{12}$

That mathematics should be so suited to such a great range of tasks is in itself quite remarkable. The mathematical physicist Eugene Wigner, in a rather famous paper on the applications of mathematics [Wig 60], calls this the "unreasonable effectiveness of mathematics" and speaks almost mystically of this as a "miracle". In this thesis I'll have very little to say about why mathematics is so remarkably useful in such a huge variety of applications. This is not because such questions are uninteresting or that they are easily answered. On the contrary, I believe that explaining the applicability of mathematics is one of the most perplexing issues in the philosophy of mathematics - it's just that a discussion of this issue would take us too far from the task at hand and, what is more, such a discussion would have little bearing on this task. ${ }^{13}$ Instead, I take as a starting point the simple, undeniable fact that mathematics has such applications. In this thesis I'll be concerned largely with indispensability arguments. These arguments purport to yield conclusions about ontology based on this simple, undeniable fact.

An indispensability argument, as Hartry Field points out, "is an argument that we should believe a certain claim [...] because doing so is indispensable for certain purposes (which the argument then details)" [Fie 89, 14]. Clearly the strength of the argument depends crucially on what the as yet unspecified purpose is. For instance,

\footnotetext{
${ }^{12}$ For example, Paul Dirac was led to the discovery of anti-matter by the equations of relativistic quantum mechanics. I'll discuss this example in more detail in section 4.4.3.

${ }^{13}$ See [Wig 60, Ham 80, Ste 89, Ste 95] for further discussion on the applicability of mathematics.
} 
few would find the following argument persuasive: we should believe that whites are morally superior to blacks because doing so is indispensable for the purpose of justifying black slavery. Similarly, few would be convinced by the argument that we ought to believe that God exists because to do so is indispensable to the purpose of enjoying a healthy religious life. The "certain purposes" of which Field speaks must be chosen very carefully. Although the two arguments above count as indispensability arguments, they are implausible because "enjoying a healthy religious life" and "justifying black slavery" are not the right sort of purposes to ensure the cogency of the respective arguments. This raises the very interesting question: which purposes are the right sort for cogent arguments?

I know of no easy answer to this question, but fortunately an answer is not required for a defence of the class of indispensability arguments with which I will be concerned in this dissertation. I will restrict my attention largely to arguments which address indispensability to our best scientific theories. I will argue that this is the right sort of purpose for cogent indispensability arguments. I will also be primarily concerned with indispensability arguments in which the "certain claim" of which Field speaks in the above passage, is an existence claim. For my purposes, then, a (scientific) indispensability argument may be defined as follows:

Argument 1 (Scientific Indispensability Argument) If apparent reference to some entity (or class of entities) $\xi$ is indispensable to our best scientific theories, then we ought to believe in the existence of $\xi$.

In the above formulation, the purpose, if you like, is that of doing science. This is a rather ill-defined purpose, and I deliberately leave it ill-defined for the moment. But to give an example of one particularly important scientific indispensability argument, with a well defined purpose, consider the argument that takes providing explanations of empirical facts as its purpose. I'll call such an argument an explanatory indispensability argument.

Although indispensability arguments are typically associated with realism about mathematical objects, it's important to realise that they do have a much wider usage. What is more, this wider usage is fairly uncontroversial. To see this we need only consider a couple of examples of explanatory indispensability arguments used for non-mathematical purposes.

Before the theory of evolution was developed, it was generally considered that the best explanation for many empirical facts about the various life forms on this planet was that these life forms were designed by God. Such an argument is a type of 
indispensability argument. Although it is now generally believed, at least amongst scientifically minded people, that God is not required for the explanation of these facts, this is simply because evolutionary theory provides a better explanation of these same facts. Thus we see a typical indispensability argument and a typical way of resisting its conclusion-find a better explanation of the facts in question that makes no appeal to the entity in question. But I'm getting ahead of myself here; for the moment I'll stick to the task of discussing what an indispensability argument is.

Take another example. Most astronomers are convinced of the existence of so called "dark matter" to explain certain facts about the rotation curves ${ }^{14}$ of spiral galaxies (amongst other things). Again this is an indispensability argument. Anyone unconvinced of the existence of dark matter is not unconvinced of the cogency of the general form of the argument being used; it's just that they are inclined to think that there are better explanations for the facts in question.

It's not too hard to see that this form of argument is very common in both scientific and everyday usage. Indeed, when presented as in the examples above, it amounts to no more than an application of inference to the best explanation. This is not to say, of course, that inference to the best explanation is completely uncontroversial. Philosophers such as Bas van Fraassen [vanF 80] and Nancy Cartwright [Cart 83] reject unrestricted usage of this style of inference. Typically, rejection of inference to the best explanation results in some form of anti-realism (anti-realism, or at least agnosticism, about theoretical entities in van Fraassen's case and antirealism about scientific laws in Cartwright's case). Such people will have little sympathy for indispensability arguments. Scientific realists, on the other hand, are generally committed to inference to the best explanation and are the main target of this dissertation. ${ }^{15}$ Indispensability arguments about mathematics urge scientific realists to place mathematical entities in the same ontological boat as (other) theoretical entities. That is, it invites them to embrace Platonism.

The use of indispensability arguments for defending mathematical realism is usually associated with Quine [Qui 48, Qui 51, Qui 60a, Qui 63, Qui 69b, Qui 81b] and Putnam [Put 71] but it's important to realise that the argument goes back much further. Gottlob Frege, for example, considers the difference between games such as chess and arithmetic and concludes that "it is applicability alone which elevates

\footnotetext{
${ }^{14}$ These are graphs of radial angular speed versus mean distance from the centre of the galaxy for stars in a particular galaxy.

${ }^{15}$ Indeed, one of the most persuasive arguments for scientific realism is generally taken to appeal to inference to the best explanation. This argument is due to J.J.C. Smart and is presented in [Sma 63].
} 
arithmetic from a game to the rank of a science" [Fre 70, 187]. As Michael Dummett points out [Dum 91, 60], Frege's appeal to the applications of arithmetic here is made in order to raise a problem for formalists who liken mathematics to a game in which mathematical symbols have no meaning, but are simply manipulated in accordance with certain rules. Frege asks the formalists to explain how such a game could have applications [Fre 70, 187]. This is clearly some form of indispensability argument.

Kurt Gödel also appeals to what is apparently an indispensability argument in 'What is Cantor's Continuum Problem?' [Göd 47]. Gödel considers reasons for adopting a new axiom of set theory and suggests that

... a probable decision about [the proposed new axiom's] truth is possible [...] by studying its "success." Success here means fruitfulness in consequences, in particular in "verifiable" consequences. [Göd 47, 477]

A little later he suggests that if the axiom in question proved to be sufficiently fruitful, then it "would have to be accepted at least in the same sense as any wellestablished physical theory" [Göd 47, 477]. The applications Gödel has in mind are intra-mathematical, but his argument is unmistakably an argument from applications to (probable) truth, which is enough for it to be called an indispensability argument.

It's no coincidence that both Frege and Gödel were mathematical realists, although neither seemed to base their realism entirely on the applications of mathematics. On the other hand, it seems they were both sensitive to the importance of the applicability of mathematics when considering mathematical truth and ontology. Frege's and Gödel's apparent endorsement of some form of indispensability argument is important in that it underlines the point that indispensability arguments for mathematical entities enjoy a wider usage than is usually realised. These arguments are not simply artifacts of the Quinean world-view. Although the form of the argument that I favour is essentially the original Quinean argument, part of my task in this thesis is to disentangle this argument from the rest of the Quinean philosophical web. I will argue that while the argument does depend on a couple of Quinean doctrines (namely, confirmational holism and naturalism), it does not depend on acceptance of all of Quine's views on science and language. In particular, it does not depend on his controversial views about indeterminacy of translation and meaning. 


\subsubsection{The Quine/Putnam Indispensability Argument}

Quine's version of the indispensability argument is to be found in many places. For instance, in 'Success and Limits of Mathematization' he says:

Ordinary interpreted scientific discourse is as irredeemably committed to abstract objects-to nations, species, numbers, functions, sets-as it is to apples and other bodies. All these things figure as values of the variables in our overall system of the world. The numbers and functions contribute just as genuinely to physical theory as do hypothetical particles. [Qui 81b, 149-150]

Here he draws attention to the fact that abstract entities, in particular mathematical entities, are as indispensable to our scientific theories as the theoretical entities of our best physical theories. Elsewhere [Qui 51, 45] he suggests that anyone who is a realist about theoretical entities, but anti-realist about mathematical entities has a "double standard" in their ontology. For instance, Quine points out that the position that scientific claims, but not mathematical claims, are supported by empirical data is untenable.

The semblance of a difference in this respect is largely due to overemphasis of departmental boundaries. For a self-contained theory which we can check with experience includes, in point of fact, not only its various theoretical hypotheses of so-called natural science but also such portions of logic and mathematics as it makes use of. [Qui 63, 367]

He is claiming here that those portions of mathematical theories that are employed by empirical science enjoy whatever empirical support the scientific theory as a whole enjoys. (I will have more to say on this matter in chapter 6.)

Hilary Putnam also once endorsed this argument:

[Q] uantification over mathematical entities is indispensable for science, both formal and physical; therefore we should accept such quantification; but this commits us to accepting the existence of the mathematical entities in question. This type of argument stems, of course, from Quine, who has for years stressed both the indispensability of quantification over mathematical entities and the intellectual dishonesty of denying the existence of what one daily presupposes. [Put 71, 347]

He elaborates on this "intellectual dishonesty" in 'What is Mathematical Truth?': 
It is like trying to maintain that God does not exist and angels do not exist while maintaining at the very same time that it is an objective fact that God has put an angel in charge of each star and the angels in charge of each of a pair of binary stars were always created at the same time! [Put 79a, 74]

Both Quine and Putnam, in the above quoted passages, stress the indispensability of mathematics to science. It thus seems reasonable to take science, or at least whatever the goals of science are, as the purpose for which mathematical entities are indispensable. But, as Putnam also points out [Put 71, 355], it is doubtful that there is a single unified goal of science. (The goals include explanation, prediction, retrodiction and so on.) Thus we see that we may construct a variety of indispensability arguments, all based on the various goals of science. As we've already seen, the explanatory indispensability argument is one important argument of this style but it is important to bear in mind that it is not the only one.

To state the Quine/Putnam indispensability argument, we need merely replace ' $\xi$ ' in argument 1 with 'mathematical entities'. For convenience of future reference I will state the argument here in a rather explicit form.

\section{Argument 2 (The Quine/Putnam Indispensability Argument)}

1. We ought to have ontological commitment to all and only those entities that are indispensable to our best scientific theories.

2. Mathematical entities are indispensable to our best scientific theories.

3. We ought to have ontological commitment to mathematical entities.

There are a number of questions about this argument that need to be addressed. The first is: The conclusion has normative force and clearly this normative force originates in the first premise, but why should an argument about ontology be normative? This question is easily answered, for I take most questions about ontology to be really questions about what we ought to believe to exist. The Quine/Putnam indispensability argument, as I've presented it, certainly respects this view of ontology. Indeed, I take it that indispensability arguments are essentially normative. For example, if you try to turn the above Quine/Putnam argument into a descriptive argument, so that the conclusion is that mathematical entities exist, you find you must have something like 'All and only those entities that are indispensable to our best theories exist' as the crucial first premise. This premise, it seems to me, is much 
more controversial than the normative one. ${ }^{16}$ As we shall see in the next chapter, this normativity arises in the doctrine of naturalism on which I will have a great deal more to say.

The next question is: How are we to understand the phrase 'indispensable to our best scientific theory'? In particular, what does 'indispensable' mean in this context? Much hangs on this question, and I'll need to treat it in some detail. I'll do this in chapter 4. (In section 4.2 I give a precise account of indispensability.) In the meantime, take it to intuitively mean 'couldn't get by without' or some such. In fact, whatever sense it is in which electrons, neutron stars, and viruses are indispensable to their respective theories will do. ${ }^{17}$

The final question is: Why believe the first premise? That is, why ought we believe in the existence of entities indispensable to our best scientific explanations? Answering this question is not easy, and an attempt to answer it will occupy the best part of the following two chapters. Briefly, I will argue that the crucial first premise follows from the doctrines of naturalism and holism. Before I embark on this task I should point out that the first premise, as I've stated it, is a little stronger than required. In order to gain the given conclusion all that is really required in the first premise is the 'all' not the 'all and only'. I include the 'all and only', however, for the sake of completeness, and also to help highlight the important role naturalism plays in questions about ontology, since it is naturalism that counsels us to look to science and nowhere else for answers to ontological questions.

As I've already made clear, I'll have more to say about naturalism and holism in the next two chapters, but it will be useful here to give an outline of the argument from naturalism and holism to the first premise of argument 2. Naturalism, for Quine at least, is the philosophical doctrine that there is no first philosophy and the philosophical enterprise is continuous with the scientific enterprise. What is more, science, thus construed (i.e. with philosophy as a continuous part) is taken to be the complete story of the world. This doctrine arises out of a deep respect for scientific methodology and an acknowledgement of the undeniable success of this methodology as a way of answering fundamental questions about all nature of things. As

\footnotetext{
${ }^{16}$ Although, arguably, once one accepts naturalism, which rules out scepticism about science, the descriptive conclusion does follow [Res 95, 171-2]. For the most part I'll ignore this and continue to talk about what we ought to believe to exist rather than what exists. See footnote 18 in this chapter.

${ }^{17}$ If you think that there is no sense in which electrons, neutron stars, and viruses are indispensable to their respective theories then the indispensability argument is unlikely to have any appeal!
} 
Quine suggests, its source lies in "unregenerate realism, the robust state of mind of the natural scientist who has never felt any qualms beyond the negotiable uncertainties internal to science" [Qui 81a, 72]. For the metaphysician this means looking to our best scientific theories to determine what exists, or, perhaps more accurately, what we ought to believe to exist. Naturalism, in short, rules out unscientific ways of determining what exists. For example, I take it that naturalism would rule out believing in the transmigration of souls for mystical reasons. It would not, however, rule out the transmigration of souls if this were required by our best scientific theories.

Naturalism, then, gives us a reason for believing in the entities in our best scientific theories and no other entities. Depending on exactly how you conceive of naturalism, it may or may not tell you whether to believe in all the entities of your best scientific theories. I take it that naturalism does give us some reason to believe in all such entities, but that this is defeasible. This is where the holism comes to the fore; in particular, confirmational holism. Confirmational holism is the view that theories are confirmed or disconfirmed as wholes. So, if a theory is confirmed by empirical findings, the whole theory is confirmed. In particular, whatever mathematics is made use of in the theory is also confirmed. Furthermore, as Putnam has stressed [Put 71], it is the same evidence that is appealed to in justifying belief in the mathematical components of the theory that is appealed to in justifying the empirical portion of the theory (if indeed the empirical can be separated from the mathematical). Taking naturalism and holism together, then, we have the first premise of argument 2 .

Before concluding this chapter, I would like to outline a couple of other indispensability arguments for mathematical entities. Although these arguments won't play any significant role in this thesis, it is, nevertheless, useful to see that the Quine/Putnam argument is not the only argument of this general form that purports to deliver mathematical realism.

\subsubsection{Other Indispensability Arguments}

I've already mentioned Frege's and Gödel's indispensability arguments. I've also outlined how one can construct various indispensability arguments that revolve around the various aims of science. For instance, we could easily construct an argument which relied on quantification over mathematical entities being indispensable for explanations. We could do the same for empirical predictions, retrodictions and so 
on. In this section I would like to outline two slightly different forms of argument that deliver Platonism as their conclusions. The first of these is Michael Resnik's pragmatic indispensability argument. The other is what I will call a semantic indispensability argument.

Michael Resnik's pragmatic indispensability argument [Res 95] focuses on the purpose of "doing science" and is a response to some problems raised for the Quine/Putnam indispensability argument by Penelope Maddy and Elliott Sober. Although I won't discuss these problems here (I do so in chapters 5 and 6), one point is important in understanding Resnik's motivation. This is that he wishes to avoid the reliance on confirmational holism that the Quine/Putnam argument requires.

Resnik states the argument in two parts. The first is an argument for the conditional claim that if we are justified in drawing conclusions from and within science, then we are justified in taking mathematics used in science to be true. He states this part of the argument as follows:

1) In stating its laws and conducting its derivations science assumes the existence of many mathematical objects and the truth of much mathematics.

2) These assumptions are indispensable to the pursuit of science; moreover, many of the important conclusions drawn from and within science could not be drawn without taking mathematical claims to be true.

3) So we are justified in drawing conclusions from and within science only if we are justified in taking the mathematics used in science to be true. [Res 95, 169-170]

He then combines the conclusion of this argument with the argument that we are justified in drawing conclusions from and within science since this is the only way we know of doing science and that we are justified in doing science. The conclusion, then, is that we are justified in taking whatever mathematics is used in science to be true. ${ }^{18}$

This argument clearly fits the mould of the scientific indispensability argument that I outlined earlier. It differs from the Quinean argument in that it doesn't rely on confirmational holism. Resnik pin-points the difference rather nicely in the following passage:

\footnotetext{
${ }^{18}$ In fact, Resnik draws the additional (stronger) conclusion that mathematics is true, arguing that this follows from the weaker conclusion since to assent to the weaker conclusion while denying the stronger invites a kind of Moore's paradox. (Moore's paradox is the paradox of asserting " $P$ but I don't believe $P$ ".)
} 
This argument is similar to the confirmational argument except that instead of claiming that the evidence for science (one body of statements) is also evidence for its mathematical components (another body of statements) it claims that the justification for doing science (one act) also justifies our accepting as true such mathematics as science uses (another act). [Res 95, 171]

This argument has some rather attractive features. For instance, since it doesn't rely on confirmational holism, it doesn't require confirmation of any scientific theories in order for belief in mathematical objects to be justified. Indeed, even if all scientific theories were disconfirmed we would (presumably) still need mathematics to do science, and since doing science is justified we would be justified in believing in mathematical objects. This is clearly a very powerful argument and one with which I have considerable sympathy. Although this argument will receive very little attention in the remainder of this thesis, it is important to see that a cogent form of argument in the general spirit of the Quine/Putnam argument can be maintained without recourse to confirmational holism.

The second argument I wish to consider in this section represents more of a departure from the Quine/Putnam argument. It does not even conform to the general pattern of a scientific indispensability argument. ${ }^{19}$ This argument takes semantics as the purpose for which mathematical entities are indispensable. According to this argument, the best candidate for a uniform semantics for our everyday discourse, our scientific discourse and our mathematical discourse is Platonism. This argument originates from Benacerraf's challenge to the philosophy of mathematics in [Ben 73]. Although the details vary, many philosophers have argued for Platonism along roughly these lines. ${ }^{20}$

In his famous paper 'Mathematical Truth' Benacerraf pointed out:

A theory of truth for the language we speak, argue in, theorize in, mathematize in, etc., should [...] provide similar truth conditions for similar sentences. The truth conditions assigned to two sentences containing quantifiers should reflect in relatively similar ways the contribution made by the quantifiers. Any departure from a theory thus homogeneous would have to be strongly motivated to be worth considering. [Ben 73, 404].

\footnotetext{
${ }^{19}$ In fact, it is not usually taken to be an indispensability argument at all. I hope, however, that it will become apparent that this argument is indeed an indispensability argument.

${ }^{20}$ For example, Crispin Wright [Wri 83], Bob Hale [Hal 87], Steven Wagner [Wag 96], Ed Zalta and Bernard Linsky [Lin 9?, Lin 95]. It might also be argued that Meinong and Frege argued for Platonism along these lines.
} 
For example, consider the following sentences:

1. There's a city larger than Melbourne.

2. There's a number larger than 17.

Both of these have the logical form $(\exists x)(F x \& L x a)$ and, what is more, both are apparently true. We would thus expect similar accounts of how each of these sentences comes to be true. (What Benacerraf is asking for is not just the correct distribution of truth values across the language, but a homogeneous story about why each sentence has the truth value it does.) The only account known to do this is Tarski's (or so the argument goes), and this is a referential semantics. This suggests that Platonism is supported by considerations of semantics.

It would certainly be a misconstrual of Benacerraf's purpose to claim that he intended this as a positive argument for Platonism. Nevertheless, such considerations do suggest a semantic argument for Platonism. Steven Wagner, for one, proposes that:

Systematic accounts of truth or validity must apparently treat predicates and logical operators as denoting various abstracta. Thus any language in which we recognize truth or valid inference yields a Platonic universe corresponding to its expressive power. [Wag 96, 76]

I take a general semantic indispensability argument to be something like the following:

Argument 3 (Semantic Indispensability Argument) If apparent reference to some entity (or class of entities) $\xi$ is indispensable to our best semantic theories of natural (and scientific) language, then we ought to believe in the existence of $\xi$. Abstracta are indispensable to our best semantic theory of natural (and scientific) language. Thus we ought to believe in such abstracta.

Although I don't wish to discuss such arguments in any detail, a few comments are warranted. Firstly, semantic indispensability arguments typically yield more abstract entities than scientific indispensability arguments. The latter only provide enough abstract entities to satisfy the needs of science, while the former provide all those and many more besides. For example, semantic considerations seem to require an abstract object for every predicate in the natural language in question. 
This highlights one of the strengths (according to some) of this style of argumentone does not have the problem of not enough abstracta. ${ }^{21}$ On the other hand, this is seen by others to be one of the weaknesses of this style of argument-we get an extremely inflated ontology far too easily. ${ }^{22}$ This leads to another weakness of this style of argument:

The problem is that science has clearer credentials than formal semantics. Physics is acceptable beyond doubt. If it admits no nominalistic construal, then Platonism is true. Semantics, however, is not clearly science and not clearly anything else that compels belief in its ontology. [Wag 96, 77]

Finally, it should be pointed out that it might be possible to combine the semantic and scientific indispensability arguments in such a way as to yield a plausible hybrid. ${ }^{23}$ Nothing prevents mathematical entities from being indispensable for multiple purposes.

I will leave the discussion of general indispensability arguments there. I hope it is clear, however, that there is nothing peculiar about the Quine/Putnam argumentit is just one of many such arguments. Indispensability arguments are not purely an artifact of the Quinean philosophical picture. In particular, many mathematical indispensability arguments may be found in the literature. This is not to say, however, that the Quine/Putnam version of the argument doesn't rely on some substantial Quinean doctrines. As we've already seen, this argument does rely on holism and (Quinean) naturalism. I'll have more to say about these in the chapters ahead. Let me now outline my strategy for the remainder of this dissertation.

\footnotetext{
${ }^{21}$ This problem seems to arise with scientific indispensability arguments-any mathematical entities that do not find applications in empirical science do not gain admission into the ontology. What is more, Bernard Linsky points out, that according to the Quine/Putnam indispensability argument, "[w]e believe in [mathematical entities] because we can't avoid it, not because we want to" [Lin 9?]. He goes on to suggest that "one would like a less grudging acceptance of the abstract" [Lin 9?].

${ }^{22}$ Sentences such as 'The round square is round' seem to require the existence of a round square! To borrow a phrase from Bertrand Russell this "has many advantages; they are the same as the advantages of theft over honest toil" [Rus 20,71].

${ }^{23}$ Indeed, Wagner suggests such a position in [Wag 96].
} 


\subsection{The Road Ahead}

As I've already stated, I will be largely concerned with defending the Quine/Putnam version of the indispensability argument against some of its detractors. ${ }^{24}$ In order to do this it will be necessary to defend some of the Quinean framework required to support the argument. In particular, I will need to defend confirmational holism and Quinean naturalism. While both these doctrines are important, naturalism is crucial. It turns out that a very specific form of naturalism is required to support the argument and, what is more, if another popular form of naturalism is substituted in place of the Quinean variety, we find the argument is without any force. Even worse, this other form of naturalism entails the non-existence of abstract entities! So rather than yielding a pro-Platonism conclusion, this other form of naturalism rules out many Platonist philosophies of mathematics. For these reasons it's clear that a fairly detailed defence of Quinean naturalism is in order. This I undertake in the following two chapters.

In chapter 2 I defend confirmational holism and begin the defence of naturalism. I isolate the crucial difference between the two versions of naturalism at issue: Quinean naturalism does not subscribe to a principle which is endorsed by the rival naturalism. This principle I call the Eleatic Principle and is (roughly) that we ought not believe in causally idle entities. I will devote all of chapter 3 to a discussion of this important principle, concluding that, despite the principle's intuitive plausibility, it is without justification. While not exactly a positive argument for Quinean naturalism, the discussion of chapters 2 and 3 does leave Quinean naturalism looking like the most plausible version of naturalism on the market.

In chapters 4, 5 and 6 I discuss some of the criticisms that the Quine/Putnam indispensability argument has attracted and I defend the argument against these criticisms. Chapter 4 is devoted to the influential work of Hartry Field who argues that mathematics is not indispensable to our best scientific theories. Furthermore, he provides an original and plausible response to the problem of explaining applicability of mathematics. I argue, in reply, that despite his impressive efforts toward showing that mathematics is dispensable to science, he has not yet done enough to undermine the second premise of argument 2 (page 11).

In chapter 5 I discuss some objections due to Penelope Maddy. Maddy raises

\footnotetext{
${ }^{24}$ Given that the Quine/Putnam indispensability argument is my primary interest in this thesis, I'll often refer to it simply as 'the indispensability argument'. Also I will often speak of 'indispensability theory' by which I will mean the Quine/Putnam indispensability argument along with that portion of Quinean philosophy required to support this argument.
} 
problems for reconciling Quinean naturalism with confirmational holism, her main point being that naturalism authorises the legitimacy of scientific methodology and this methodology does not support confirmational holism. Chapter 6 is dedicated to defending one of the less intuitive consequences of the indispensability argument: that mathematical knowledge has an empirical character.

In the first six chapters I do not discuss at any length the details of the Platonism that the indispensability argument yields. In chapter 7 I explain why. I show that although Quine had his own favoured account of what mathematical objects are, his argument can be used to defend a surprising variety of Platonist positions. I also address the important issue of how such accounts reply to the Benacerraf challenges mentioned at the beginning of chapter 1. Finally, I look at uses of indispensability arguments outside the philosophy of mathematics and consider the worry that indispensability arguments can be used to justify too much.

I also include two appendices. The first provides a chapter-by-chapter summary, while the second provides a summary of the technical notation I employ. 



\section{Chapter 2}

\section{The Quinean Backdrop}

In this chapter I will discuss the doctrines required to support the Quine/Putnam indispensability argument. Although a great deal of Quine's philosophy is interconnected, making the isolation of particular doctrines very difficult, I will argue that the two essential theses for our present purposes-confirmational holism and naturalism - can be disentangled from the rest of the Quinean web. What is more, I will show that these two theses do not depend in any significant way on some of Quine's more controversial views about the philosophy of language.

In the first section I will discuss two different ways in which naturalism is commonly construed, and how each leads to different answers to the important question of the ontological status of mathematical entities. In the next section I shall outline the Quinean sense of naturalism, identifying what sets it apart from other varieties of naturalism. In section 2.3 I shall defend this conception of naturalism against the charge that it fails to recognise an important methodological difference between science and philosophy. In the following section I discuss another variety of naturalism, and I will identify the salient difference between this and the Quinean variety in terms of a causal test which I call the Eleatic Principle: Quinean naturalism rejects this test while the other, causal, version of naturalism accepts it. In section 2.5, I will discuss confirmational holism and its importance to the indispensability argument. Finally, I will discuss how holism and naturalism, as I've presented them, support the crucial first premise of the Quine/Putnam indispensability argument (argument 2, page 11). 


\subsection{Introducing Naturalism}

Naturalism, in its most general form, is the doctrine that we ought to seek accounts of the nature of reality that are not "other-worldly" or "unscientific", but to be more precise than this is to immediately encounter trouble. For instance, in Philosophical Naturalism, David Papineau points out that "nearly everybody nowdays wants to be a 'naturalist', but the aspirants to the term nevertheless disagree widely on substantial questions of philosophical doctrine" [Pap 93, 1]. In one way this is not at all surprising, for after all, there is no compulsion for all naturalists to agree on other philosophical stances, distinct from naturalism, and such stances, when combined with naturalism, presumably yield widely different results. It all depends on what you mix your naturalism with.

There is, however, another reason for disagreement amongst naturalistic philosophers: different philosophers use the word 'naturalism' to mean different things. Naturalism involves a certain respect for the scientific enterprise - that much is common ground-but exactly how this is cashed out is a matter of considerable debate. For instance, for David Armstrong naturalism is the doctrine that "nothing but Nature, the single, all-embracing spatio-temporal system exists" [Arm 78, Vol. 1, 138], ${ }^{1}$ whereas, for Quine, naturalism is the "abandonment of the goal of a first philosophy" [Qui 81a, 72]. ${ }^{2}$

One issue on which naturalistic philosophers disagree widely, and which is of fundamental importance in this dissertation, is the ontological status of mathematical entities. We saw in the last chapter how the Quine/Putnam indispensability argument legitimates belief in mind-independent mathematical objects, and that this argument depends on naturalism. On the other hand, philosophers such as David Armstrong cite naturalism as grounds for rejecting belief in any such mindindependent abstract objects. ${ }^{3}$

While there is no way of preventing philosophers from mixing their naturalism with other philosophical doctrines (so long as the mix is consistent) there is good

${ }^{1}$ This conception of naturalism has resonances in D.C. Williams's materialism [Wil 44]. I am grateful to Bernard Linsky for pointing out this reference in his [Lin 9?].

${ }^{2}$ Quine's conception of naturalism also has important predecessors. For example, Bertrand Russell identifies the new philosophical movement he calls 'realism' with the abandonment of "the claim to a special philosophic method or a peculiar brand of knowledge to be obtained by its means" [Rus 24,69]. He goes on to say that this new philosophy "regards philosophy as essentially one with science" and that "[i]t conceives that all knowledge is scientific knowledge" [Rus 24, 69-70]. Again I'm indebted to Linsky [Lin 9?] for this reference.

${ }^{3}$ I will look at Armstrong's rejection of abstract objects later in this chapter (section 2.4), and in considerably more detail in the next chapter. 
reason for requiring that the various, often contradictory, positions that fly under the banner of naturalism be disentangled, from one another. This is an extremely complicated matter but in the next three sections I make some progress towards this goal. At the very least I hope to identify the difference between the varieties of naturalism which may be used to undermine mathematical realism and the Quinean variety.

\subsection{Quinean Naturalism}

Quine's aphoristic characterisations of naturalism are well known. In 'Five Milestones of Empiricism' he tells us that naturalism is the

abandonment of the goal of a first philosophy. It sees natural science as an inquiry into reality, fallible and corrigible but not answerable to any suprascientific tribunal, and not in need of any justification beyond observation and the hypothetico-deductive method. [Qui 81a, 72]

And that:

[t] he naturalistic philosopher begins his reasoning within the inherited world theory as a going concern. He tentatively believes all of it, but believes also that some unidentified portions are wrong. He tries to improve, clarify, and understand the system from within. He is the busy sailor adrift on Neurath's boat. [Qui 81a, 72]

The aphorisms are useful but they also mask a great deal of the subtlety and complexity of Quinean naturalism. Indeed, the subtleties and complexities of naturalism are far greater than one would expect for such a widely held and intuitively plausible doctrine. We would thus be well served spending a little time coming to better understand Quinean naturalism.

As I see it, there are two strands to Quinean naturalism. The first is a normative thesis concerning how philosophy ought to approach certain fundamental questions about our knowledge of the world. The advice here is clear: look to science (and nowhere else) for the answers. Science, although incomplete and fallible, is taken to be the best guide to answering all such questions. In particular, "first philosophy" is rejected. That is, Quine rejects the view that philosophy precedes science or oversees science. This thesis has implications for the way we should answer metaphysical questions, for instance: we should determine our ontological commitments 
by looking to see what entities our best scientific theories are committed to. Thus I take it that naturalism tells us (a) we ought to grant real status to only the entities of our best scientific theories and (b) we ought to (provisionally) grant real status to all the entities of our best scientific theories. For future reference I'll call this first strand of Quinean naturalism the no-first-philosophy thesis (or NFPT for short) and its application to metaphysics the Quinean ontic thesis (or QOT for short).

It is worth pointing out that QOT is distinct from a thesis about how we determine the ontological commitments of theories. According to this thesis, the ontological commitments of theories are determined on the basis of the domain of quantification of the theory in question. ${ }^{4}$ Call this thesis the ontological commitments of theories thesis $(O C T T)$. One could quite reasonably believe OCTT without accepting $Q O T$. For instance, I take it that Bas van Fraassen is such a person. He accepts that our current physics is committed to entities such as electrons and the like, but it does not follow that he believes that it is rational to believe in these entities in order to believe the theory [vanF 80,4]. OCTT is purely descriptive whereas $Q O T$ is, as I've pointed out, normative. From here on I shall be concerned only with $Q O T$, but it is worth bearing in mind the difference, because I don't think that OCTT rightfully belongs to the doctrine of naturalism. It is an answer to the question of how we determine the ontological commitments of theories, but it is not the only naturalistic way such questions can be answered.

The second strand of Quinean naturalism is a descriptive thesis concerning the subject matter and methodology of philosophy and science. Here naturalism tells us that that philosophy is continuous with science and that together they aim to investigate and explain the world around us. What is more, it is supposed that this science-philosophy coalition is up to the task. That is, all phenomena are in principle explicable by science. For future reference I'll call this strand the continuity thesis (or CT for short).

Although it is instructive to distinguish the two strands of Quinean naturalism in this way, it is also important to see how intimately intertwined they are. Firstly, there is the intriguing interplay between the two strands. NFFT tells us that we ought to believe our best scientific theories and yet, according to $C T$, philosophy is part of these theories. This raises a question about priority: In the case of a conflict between philosophy and science, which gets priority? I'll have more to say on this issue in section 5.2 , but for now it will suffice to say that philosophy does not occupy a privileged position. That much is clear. But it also appears, from the

\footnotetext{
${ }^{4}$ See [Qui 48, 12-13] for details.
} 
fact that philosophy is seen as part of the scientific enterprise, that science (in the narrow sense-i.e. excluding philosophy) occupies no privileged position either.

The second important connection between the two strands is the way in which $C T$ lends support to NFFT. The traditional way in which first philosophy is conceived is as an enterprise that is prior and distinct from science. Philosophical methods are seen to be a priori while those of science are a posteriori. But accepting $C T$ rules out such a view of the relationship between philosophy and empirical science. Once philosophy is located within the scientific enterprise it is more difficult to endorse the view that philosophy oversees science. I'm not claiming that $C T$ entails NFPT; just that it gives it a certain plausibility. ${ }^{5}$

In the discussion so far I've glossed over the question of what constitutes our best scientific theories. ${ }^{6}$ I'll have something (though not much) to say about how we answer this question in section 4.3. What is important in the meantime is that there is room for disagreement about what our best scientific theories are. This will become important in the discussion of David Armstong's version of Naturalism in section 2.4 .

Now let me say something about why one ought to embrace naturalism. I won't embark on a general defence of naturalism in this thesis - that would be far too ambitious. Instead, I shall ask that you accept, for the sake of the discussion, some suitably broad sense of this doctrine. I suggest that we accept something like the conception of naturalism that I opened section 2.1 with. For example:

We ought to seek only scientific accounts of reality.

What I need to do now is convince you of Quinean naturalism. In order to do this I'll need to identify what is distinctive about Quinean naturalism. In particular, I need to identify and justify those features of the Quinean conception of naturalism that support the crucial first premise of argument 2 on page 11.

Firstly let me mark out the common ground. Naturalists of all ilks agree that we should look only to science when answering questions about the nature of reality. What is more, they all agree that there is at least prima facie reason to accept all the entities of our best scientific theories. That is they all agree that there is a metaphysical component to naturalism. So they are inclined to accept the first part of $Q O T$ (the "only" part) and are inclined to, at least provisionally, accept the second

\footnotetext{
${ }^{5}$ Indeed, CT cannot entail NFPT since the former is descriptive and the latter normative.

${ }^{6}$ There is also the question of what constitutes a scientific theory as opposed to a non-scientific theory. I won't enter into that debate here: I'll assume that we have at least an intuitive idea of what a scientific theory is.
} 
part (the "all" part). (Most naturalists believe that naturalism entails scientific realism but they are inclined to be a little reluctant to embrace all the entities of our best scientific theories.) ${ }^{7}$ What I take to be the distinctive feature of Quinean naturalism is the view that our best scientific theories are continuous with philosophy and are not to be overturned by first philosophy. It is this feature that blocks any first-philosophy critique of the ontological commitments of science. Consequently, it is this feature of Quinean naturalism that is of fundamental importance to the indispensability argument.

Let me begin my promotion of Quinean naturalism by showing how it provides a defence against scepticism. The sceptic might ask what justification we have for postulating physical objects from the meagre input of certain two-dimensional electromagnetic irradiation patterns on our retinas. Our theories clearly outstrip our evidence for them, so it is the business of epistemology to give a factual account of the relation between the two. ${ }^{8}$ Quine points out, however, that "the skeptical challenge springs from science itself, and that in coping with it we are free to use scientific knowledge" [Qui 74, 3]. After all, the idea of being deceived about physical objects, for instance, depends on science in two ways: (i) the deception consists in believing something other than the scientific picture of the world and (ii) it is science itself that informs us that our data about the world is both incomplete and fallible. If scepticism originates within science, it is only reasonable that the epistemologist is justified in using whatever portion of science s/he requires to combat scepticism. From the point of view of Quine's naturalised epistemology, there is no more secure vantage point than the vantage point of our best scientific theories. Thus the naturalised epistemologist "no longer dreams of a first philosophy, firmer than science, on which science can be based; he is out to defend science from within, against its self doubts" [Qui 74, 3].

\subsection{The Methodologies of Philosophy and Science}

In this section I continue my defence of Quinean naturalism by defusing an objection that it faces. The objection is that Quinean naturalism (in particular $C T$ ) fails to acknowledge an important methodological difference between science and philosophy. After all, so the objection goes, it is clear that philosophy proceeds by a priori

\footnotetext{
${ }^{7}$ For example Keith Campbell advocates "selective realism" in [Cam 94] and even Quine restricts commitment to indispensable entities.

${ }^{8}$ Quine calls this relation "[t]he relation between the meager input and the torrential output" [Qui 69a, 83].
} 
methods, such as thought experiments and deduction, whereas science proper proceeds by a posteriori methods, the celebrated scientific method of hypotheses and observation, typically involving real experiments and induction. In reply I will be content to rehearse Quine's argument against the possibility of a priori knowledge. In addition to this, I wish to cast doubt on the legitimacy of characterising philosophy as proceeding by a priori methods and science as proceeding by a posteriori methods. Moreover, the discussion of this objection will help elucidate certain features of Quinean naturalism-namely its connection to confirmational holism.

\title{
2.3.1 The 'Two Dogmas' Argument
}

In his famous article 'Two Dogmas of Empiricism' Quine launches a two-fold attack on the analytic/synthetic distinction. The first part of this argument is to show that there is no non-circular definition of 'analytic'. For instance, he argued that we cannot define analyticity by way of the notion of synonymy. As Graham Priest [Prie 79] points out, however, this circularity argument is not so much a condemnation of analyticity, since many important concepts can only be defined in circular terms. ${ }^{9}$ The point here is that the circularity argument prevents a defence of analyticity by appeal to synonymy when analyticity comes under fire, because synonymy, being part of the circle, is just as much under fire as analyticity. The attack proper, then, is an argument from the history of modern science that no belief can be held onto no matter what. I can do no better than to quote Quine here.

\begin{abstract}
Any statement can be held true come what may, if we make drastic enough adjustments elsewhere in the system. Even a statement very close to the periphery can be held true in the face of recalcitrant experience by pleading hallucination or by amending certain statements of the kind called logical laws. Conversely, by the same token, no statement is immune to revision. Revision even of the logical law of excluded middle has been proposed as a means of simplifying quantum mechanics; and what difference is there in principle between such a shift and the shift whereby Kepler superseded Ptolemy, or Einstein Newton, or Darwin Aristotle? [Qui 51, 43]
\end{abstract}

The main point is that the history of science has taught us that what were once considered analytic truths, such as that Pythagoras' theorem holds in our world ${ }^{10}$

\footnotetext{
${ }^{9}$ Indeed, if Quine is correct about language, this is true of all concepts!

${ }^{10} \mathrm{By}$ this I simply mean to rule out the claim that Pythagoras' theorem holds in abstract Euclidean spaces.
} 
or that any massive body can be accelerated without bound, have been given up in order to cohere with new and better scientific theories. Thus, by an inductive argument from such examples, we conclude that there are no analytic truths. ${ }^{11}$

Putnam, in 'Two Dogmas Revisited' [Put 76], points out that the idea of an analytic truth as one which is confirmed no matter what is quite different from the original Kantian notion of "the predicate contained in the concept"; it is, in fact, much more like the traditional notion of apriority. So if we take Quine's argument from the history of science on its own, we have an argument against the a priori/a posteriori distinction. Furthermore, the argument, thus construed, does stand on its own; it does not depend on the circularity argument, which has attracted some criticism since the publication of 'Two Dogmas of Empiricism'. ${ }^{12}$ We see then that if the a priori/a posteriori distinction cannot be maintained, the claim that Quinean naturalism fails to recognise this distinction is misguided. Note, however, that for Quine some beliefs may be more central than others, and so may have an a priori "feel" to them but strictly speaking they are not a priori.

It is also worth noting explicitly that in the foregoing defence of $C T$ we have seen an important consequence of Quinean naturalism: some sort of holism about our scientific theories. The fact that we cannot distinguish between a priori and a posteriori portions of our theory and also the fact that it seems that isolated hypotheses do not enjoy empirical confirmation or disconfirmation-only bodies of hypotheses may be said to be confirmed or disconfirmed-suggests confirmational holism. While it's clear that there is a close relationship between naturalism and holism, I do not wish to take too much for granted here. I do not wish to presuppose that the confirmational holism required for the success of the indispensability argument is written into naturalism. It would be nice if it were, but I think it's safer to suppose that it is not. I will thus argue for confirmational holism separately in section 2.5. For now I'll continue my defence of Quinean naturalism against the charge that it fails to respect the methodological differences between philosophy and science.

\footnotetext{
${ }^{11}$ Perhaps the conclusion here seems a bit stronger than the argument will support, but at the very least we can conclude that even if there are such things as analytic truths, which are confirmed come what may, then we are notoriously bad at recognising them. We should thus give them no privileged place in science. This weaker conclusion is all that Quine really requires, and certainly all that I require in this present discussion.

${ }^{12}$ See, for instance, [Prie 79], [Gri 56] and [Put 76] for some of these criticisms.
} 


\subsubsection{How Useful is the Distinction?}

It seems that even if someone accepts the argument from the preceding section they might claim that Quinean naturalism fails to distinguish between the quite different methodologies of philosophy and science: the former proceeds by pseudo-apriori methods; that is, by relying on beliefs central to science; such as logic; whereas empirical science proceeds by clearly a posteriori methods. This objection, though, is again misguided. The history of science is littered with examples of science proceeding by apparently a priori methods. This is the domain of the theoretical scientist, who must tease out consequences of theories using deduction and thought experiments. To give but one example, Galileo's famous law that all bodies fall to earth with the same velocity regardless of their mass was derived, it seems, by a thought experiment which showed that the received view from Aristotelian physics (heavier objects fall more quickly than lighter ones) was inconsistent. ${ }^{13}$ Furthermore, this law flew in the face of empirical evidence, as stones were repeatedly observed to fall faster than leaves. The success of Galileo's law over the received view demonstrates that internal consistency is quite rightly seen as a more important feature of a scientific theory than empirical adequacy, and since determining internal consistency is the business of the theoretical scientist, pseudo-apriori methods are not only part of science, they are an important part of science.

The converse, however, does not seem true: philosophers do not engage in empirical inquiry. I have two things to say in relation to this. The first is that this in itself doesn't seem enough to exclude philosophy from the domain of science; after all, as we have already seen, theoretical physicists don't perform experiments either, and yet no-one wishes to bar them from the science club! The other relevant point here is that while philosophers don't engage in empirical inquiry themselves, they are certainly not unaware of developments in modern experimental science. For example, those philosophers working in philosophy of mind must keep a close eye on developments in experimental psychology, computer science and neurophysiology.

Perhaps, though, there is some fundamental difference between the philosopher's thought experiments and the scientist's. Frank Jackson suggests that one way which

\footnotetext{
${ }^{13}$ Consider two bodies, $\mathcal{B}_{1}$ and $\mathcal{B}_{2}$, where $\mathcal{B}_{1}$ is heavier than $\mathcal{B}_{2}$. If we were to tie these two bodies together with a piece of twine of sufficient strength and allow them to fall freely from the same height, Aristotelian physics would tell us: since $\mathcal{B}_{1}$ is heavier than $\mathcal{B}_{2}$ we should expect $\mathcal{B}_{1}$ to fall faster until the piece of twine becomes tight and then $\mathcal{B}_{2}$ will retard $\mathcal{B}_{1}$ 's motion so the velocity of the system will be slower than if $\mathcal{B}_{1}$ had been dropped on its own. On the other hand, the mass of the system is greater than the mass of $\mathcal{B}_{1}$ 's alone and so the system's velocity should be faster than if $\mathcal{B}_{1}$ had been dropped on its own.
} 
someone might wish to distinguish between the two is that scientific thought experiments tell us something about the world, whereas philosophical thought experiments tell us something about the way we use our language [Jac 97]. For instance, Galileo's thought experiment, mentioned above, tells us that all objects fall with the same velocity irrespective of mass, whereas Hilary Putnam's famous "twin earth" thought experiment tells us something about the way we use the term 'water'. ${ }^{14}$ This is not at all clear to me though. Surely all Galileo's thought experiment shows is that there is an inconsistency in Aristotelian physics which was rectified by dropping the proposition that heavier objects fall faster than lighter ones. The contradiction might have been resolved by altering the way we use some of the crucial words. Indeed, it is true that Galileo's law that all bodies fall with the same velocity is false unless what we mean by 'fall' is 'fall in a vacuum'. So to some extent, at least, Galileo's thought experiment does help to clarify our use of the relevant bits of language.

Perhaps a better example is Einstein's special relativity thought experiment. Much of Einstein's work in the special theory of relativity was concerned with clarifying what is meant by length, time, velocity, and simultaneity rather than telling us how the world is (although it clearly does the latter as well). Indeed, Einstein himself takes this view of at least part of his work in the 1905 paper on special relativity, as illustrated by the following passage:

Thus with the help of certain imaginary physical experiments we have settled what is to be understood by synchronous stationary clocks located at different places, and have evidently obtained a definition of "simultaneous," or "synchronous," and of "time." [Ein 05, 40]

Take for example the classical (Galilean) addition of velocities formula:

$$
V_{\mathcal{G}}=v_{a}+v_{b}
$$

and compare it with the relativistic formula

$$
V_{\mathcal{R}}=\frac{v_{a}+v_{b}}{1+\frac{v_{a} v_{b}}{c^{2}}}
$$

\footnotetext{
${ }^{14}$ Putnam invites us to consider earth and twin earth. These two worlds are exactly alike except for the following: in the former world the occupants use the word 'water' to refer to $\mathrm{H}_{2} \mathrm{O}$ and in the latter the occupants use it to refer to some other substance XYZ which is otherwise similar to $\mathrm{H}_{2} \mathrm{O}$. Putnam's (and many others') intuition is that we, occupants of earth, would not call the twin-earth substance, XYZ, 'water' [Put 73]. Putnam's conclusion is that the meaning of 'water' is not fixed entirely by the internal states of speakers.
} 
where $v_{a}$ is the velocity of some body a (moving with uniform velocity) relative to some inertial frame $\mathcal{F}, v_{b}$ is the velocity of some other body $b$ (also moving with uniform velocity) relative to $a, V_{\mathcal{G}}$ is the velocity of $b$ relative to $\mathcal{F}$ in the Galilean/Newtonian theory, $V_{\mathcal{R}}$ is the velocity of $b$ relative to $\mathcal{F}$ in the special theory of relativity, and $c$ is the speed of light in a vacuum. It is clear that in general $V_{\mathcal{G}} \neq V_{\mathcal{R}}$ and it is well known that equation (2.2) has replaced (2.1) as the correct formula for evaluating addition of velocities. ${ }^{15}$

It might well be argued that there is a change of meaning in some of the relevant terms (such as 'velocity') for such a revision of the addition of velocities formula to occur. Similarly the replacement of the classical kinetic energy and momentum formulae in special relativity indicates changes of meanings of these terms in the new theory. The moral of all this is simply that it is not at all clear that scientific thought experiments are concerned only with the way the world is. They also shed light on the way we use bits of the language of the relevant theory, and in particular, which concepts are the key ones. For instance, special relativity tells us that $V_{\mathcal{R}}$ is the important concept when adding velocities, not $V_{\mathcal{G}}$, despite the latter's intuitive appeal.

As a final attempt to maintain some sort of distinction between philosophy and science, someone might argue that Jackson's distinction between scientific thought experiments and philosophical thought experiments can be upheld; it's just that Einstein, for instance, was indulging in philosophical analysis in his 1905 paper. But surely if the distinction in question cannot be maintained by appeal to scientific and philosophical practices, then one ought to wonder whether the distinction is really picking out anything more significant than the distinction between, say, physics and chemistry. At the very least, it is clear that the boundary between philosophy and science is vague and this is enough to support the acceptance of $C T$.

To sum up then. Firstly, if Quine is right and there really is no a priori knowledge ${ }^{16}$ then there can be little substance to the thought that philosophy is a priori and science a posteriori. Secondly, even if you disagree with Quine on this, it is not at all clear that the cleavage between the a priori and the a posteriori corresponds

\footnotetext{
${ }^{15}$ Of course (2.1) is still used for velocities small in relation to $c$, but it is understood that values so obtained are estimates.

${ }^{16}$ This may not be strictly true. Hilary Putnam in 'Analyticity and Apriority: Beyond Wittgenstein and Quine' ([Put 79b]) suggests that there may be at least one a priori truth, but this is of little interest since it is not the sort of proposition that is likely to play a significant role in any scientific theory. For the record, Putnam claims that the statement 'Not every statement is both true and false' is true a priori since "to deny that statement would be to forfeit rationality itself" [Put 79b, 129].
} 
to any significant cleavage at all, let alone to the cleavage between philosophy and science. The fact that Quinean naturalism fails to respect such a cleavage is far from a deficiency of the position, it is one of its great strengths.

So, having dealt with what I think are the more obvious objections to Quinean naturalism, I now turn to the task of discussing its main rival.

\subsection{The Causal Version of Naturalism}

One interesting way in which someone can give an alternative account of naturalism is by giving an alternative account of what our best scientific theories are. ${ }^{17}$ This can be done by placing some restriction on current scientific theories, such as by believing some portion of these theories and remaining instrumental about the rest. ${ }^{18}$ This style of account is considered very seductive by many, and in this section I will consider one such account advanced by David Armstrong.

Armstrong defines naturalism "as the doctrine that reality consists of nothing but a single all-embracing spatio-temporal system" [Arm 80a, 149]. This conception of naturalism has an important consequence (at least according to Armstrong): we should believe in only causally active entities (or perhaps, more generously, potentially causally active entities). I shall refer to this as the Eleatic Principle ${ }^{19}$ or sometimes as the causal requirement. According to Armstrong, non-spatio-temporally located entities would be incapable of acting on particulars, and so can play no explanatory role in science. He concludes that we have no rational reason to postulate them. Elsewhere he admits that the latter argument is less than conclusive, but nonetheless "gives us good reason for denying the existence of such entities" [Arm 89, 7]. I will not discuss Armstrong's argument in any detail here, (I'll have more to say about it in chapter 3 , along with other justifications of this principle). For now I merely wish to draw attention to this consequence of Armstrong's

\footnotetext{
${ }^{17}$ Clearly the alternative account of what our best scientific theories are better not be too radical lest it cease to qualify as naturalistic. Construing astrology and literal readings of the Bible as our best scientific theories would not do.

${ }^{18}$ Indeed, it seems scientists themselves are instrumental about the more fanciful areas of science; that is, those which are not firmly supported by theory and experimental evidence. This instrumentalism seems to shift to realism as the area in question becomes better understood. However, I take such instrumentalism to be simply an indication that the area of theory in question isn't (yet) part of the best theory.

${ }^{19}$ It is called the Eleatic Principle after a passage from Plato's Sophist in which the Eleatic stranger suggests that causal power is the mark of being [Pla 35, 247d-e]. David Armstrong cites this passage in [Arm 78, Vol. 2, 45-46] and Graham Oddie coined the phrase 'the Eleatic Principle' in [Odd 82].
} 
naturalism.

I've already noted the disagreement between Quine and Armstrong on the ontological status of mathematical entities. For Armstrong, nominalism follows fairly directly from the Eleatic Principle. This is in stark contrast with Quinean naturalism which, as we've seen already, supports Platonism. Clearly the Eleatic Principle is the crucial difference. It is interesting, however, to ask after the status of this principle. If Armstrong is using the Eleatic Principle to overrule science on ontological matters-science being committed to mathematical entities and Armstrong ruling against them - then he is guilty of practising first philosophy. Perhaps, more plausibly, Armstrong sees the Eleatic Principle as part of our best scientific theory. Indeed, given his remarks above about his argument for the Eleatic Principle being inconclusive, this seems the more likely.

Whether the Eleatic Principle is considered part of a first philosophy or part of our best science doesn't matter too much; it's clear that Armstrong's naturalism is committed to a causal test of this kind. Either way this test will require justification and I devote the whole of the next chapter to the important task of examining the arguments put forward for it. For now let me join David Lewis in expressing general concerns about philosophers who wish to interfere with science or mathematics because of commitment to principles such as the Eleatic Principle. (Lew is is specifically speaking of those who wish to interfere with mathematics based on the view that there are no classes, but the general lesson is clear.) He says:

I am moved to laughter at the thought of how presumptuous it would be to reject
mathematics for philosophical reasons. How would you like the job of telling
the mathematicians that they must change their ways, and abjure countless
errors, now that philosophy has discovered that there are no classes? Can you
tell them, with a straight face, to follow philosophical argument wherever it
may lead? If they challenge your credentials, will you boast of philosophy's
other great discoveries: that motion is impossible, that a Being than which no
greater can be conceived cannot be conceived not to exist, that it is unthinkable
that anything exists outside the mind, that time is unreal, that no theory
has ever been made at all probable by evidence (but on the other hand that
an empirically ideal theory cannot possibly be false), that it is a wide-open
scientific question whether anyone has ever believed anything, and so on, and
on, ad nauseam? Not me! [Lew 91, 59]

Nor me! 


\subsection{Holism}

Holism comes in many forms. Even in Quine's philosophy there are at least two different holist theses. The first is what is usually called semantic holism (although Quine calls it moderate holism [Qui 81a, 71]) and is usually stated, somewhat metaphorically, as the thesis that the unit of meaning is the whole of the language. As Quine puts it:

The idea of defining a symbol in use was [...] an advance over the impossible term-by-term empiricism of Locke and Hume. The statement, rather than the term, came with Bentham ${ }^{20}$ to be recognized as the unit accountable to an empiricist critique. But what I am now urging is that even in taking the statement as unit we have drawn our grid too finely. The unit of empirical significance is the whole of science. [Qui 51, 42]

Semantic holism is closely related to Quine's denial of the analytic/synthetic distinction and his thesis of indeterminacy of translation. He argues for the former in a few places, but most notably in 'Two Dogmas of Empiricism' [Qui 51], while the latter is presented in Word and Object [Qui 60].

The other holist thesis found in Quine's writings is confirmational holism (also commonly referred to as the Quine/Duhem thesis). As Fodor and Lepore point out [Fod 92, 39-40] the Quine/Duhem thesis receives many different formulations by Quine and it is not clear that all these formulations are equivalent. For example, in Pursuit of Truth Quine writes:

$[T]$ he falsity of the observation categorical ${ }^{21}$ does not conclusively refute the hypothesis. What it refutes is the conjunction of sentences that was needed to imply the observation categorical. In order to retract that conjunction we do not have to retract the hypothesis in question; we could retract some other sentence of the conjunction instead. This is the important insight called holism. [Qui 90, 13-14] $]^{22}$

And in 'Two Dogmas of Empiricism', in a much quoted passage, he suggests that "our statements about the external world face the tribunal of sense experience not

\footnotetext{
${ }^{20}$ Interestingly, in the original version of the paper in Philosophical Review the reference here is to Russell. This was exchanged for Frege in the first edition of From a Logical Point of View and, finally, for Bentham in the second edition of From a Logical Point of View [Fod 92, 216].

${ }^{21} \mathrm{By}$ 'observation categorical' Quine simply means a statement of the form 'whenever $P$, then $Q$ '. For example, 'where there's smoke, there's fire'.

${ }^{22}$ Cf. [Duh 06, 187] for a similar statement of the thesis by Duhem.
} 
individually but only as a corporate body" [Qui 51, 41]. In a similar vein in "On Mental Entities' he tells us:

As Pierre Duhem urged, it is the system as a whole that is keyed to experience. It is taught by exploitation of its heterogeneous and sporadic links with experience, and it stands or falls, is retained or modified, according as it continues to serve us well or ill in the face of continuing experience. [Qui 53a, 222]

In the last two of these three passages Quine emphasizes the confirmational aspects of holism-it's the whole body of theory that is tested; not isolated hypotheses. In the first passage he emphasizes disconfirmational aspects of holism-when our theory conflicts with observation any number of alterations to the theory can be made to resolve the conflict. Despite the difference in emphasis, I take it that these two theses are equivalent (or near enough). Moreover, I take it that they are both true, modulo some quibbles about how much theory is required to face the tribunal at any time.

It's somewhat ironic that Quine argues for confirmational holism (which I, and many others, take to be a relatively uncontroversial thesis) from his semantic holism, which is one of the most controversial parts of Quine's philosophy. The debate about the rejection of the analytic/synthetic distinction, in particular, is still raging some 46 years after the publication of 'Two Dogmas of Empiricism'. I don't deny that confirmational holism does follow from semantic holism; it's just that there are easier, less controversial roads to confirmational holism. Since it's only confirmational holism that we require for the indispensability argument, I intend to explore these other roads and thus avoid the semantic holism debate.

Both Duhem [Duh 06] and Lakatos [Lak 70a] have argued for confirmational holism without any (obvious) recourse to semantic considerations. They emphasize the simple yet undeniable point that there is more than one way in which a theory, faced with recalcitrant data, can be modified to conform with that data. Consequently certain core doctrines of a theory may be held onto in the face of recalcitrant data by making suitable alterations to auxiliary hypotheses. This point is driven home by appeal to case studies from the (actual and imagined) history of science. Indeed, such is the influence of Duhem, Lakatos and Quine on this point that nowdays few require convincing. I will, however, present one example to illustrate their point. This example is due to Lakatos [Lak 70a] and is quoted by Chalmers in [Cha 82, 65-66]. I too will let Lakatos tell the story. 
The story is about an imaginary case of planetary misbehaviour. A physicist of the pre Einsteinian era takes Newton's mechanics and his law of gravitation, $\mathrm{N}$, the accepted initial conditions, I, and calculates, with their help, the path of a newly discovered small planet, $p$. But the planet deviates from the calculated path. Does our Newtonian physicist consider that the the deviation was forbidden by Newton's theory and therefore that, once established, it refutes the theory N? No. He suggests that there must be a hitherto unknown planet $p^{\prime}$, which perturbs the path of $p$. He calculates the mass, orbit, etc. of this hypothetical planet and then asks an experimental astronomer to test his hypothesis. The planet $p^{\prime}$ is so small that even the biggest available telescopes cannot possibly observe it; the experimental astronomer applies for a research grant to build yet a bigger one. In three years time, the new telescope is ready. Were the unknown planet $p^{\prime}$ to be discovered, it would be hailed as new victory of Newtonian science. But it is not. Does our scientist abandon Newton's theory and his idea of the perturbing planet? No. He suggests that a cloud of cosmic dust hides the planet from us. He calculates the location and properties of this cloud and asks for a research grant to send up a satellite to test his calculations. Were the satellite's instruments (possibly new ones, based on a little-tested theory) to record the existence of the conjectural cloud, the result would be hailed as an outstanding victory for Newtonian science. But the cloud is not found. Does our scientist abandon Newton's theory, together with the idea of the perturbing planet and the idea of the cloud which hides it? No. He suggests that there is some magnetic field in that region of the universe which disturbs the instruments of the satellite. A new satellite is sent up. Were the magnetic field to be found, Newtonians would celebrate a sensational victory. But it is not. Is this regarded as a refutation of Newtonian science? No. Either yet another ingenious auxiliary hypothesis is proposed or ... the whole story is buried in a dusty volume of periodicals and the whole story never mentioned again. [Cha 82, 65-66]

The story, although humourous in tone, does illustrate how much theory is employed for what might seem like straight-forward scientific observations. Thus, the unit of confirmation or disconfirmation is not the single hypothesis but, rather, some substantial body of hypotheses. Moreover, a fairly good case can be made for thinking that in some instances this larger body of hypotheses is the whole of science. 
Before leaving the doctrine of holism, I wish to consider one last question: Might one accept holism as stated, but reject the claim that mathematical propositions are one with the rest of science? That is, might it not be possible to pin-point some semantic difference between the mathematical propositions employed by science and the rest, with empirical confirmation and disconfirmation reserved for the latter? Carnap [Carn 37], with his appeal to "truth by convention", suggested exactly this. Quine of course denies that this can be done [Qui 36, Qui 63, Qui 51], but the reasons for his denial would take us deep into semantic holism. For our present purposes, it will suffice to note that there is no obvious way of disentangling the purely mathematical propositions from the main body of science. Our empirical theories have the so-called empirical parts intimately intertwined with the mathematical. A cursory glance at any physics book will confirm this, where one is likely to find "mixed statements" such as: 'planets travel in elliptical orbits'; 'the curvature of space-time is not zero'; 'the work done by the force on the particle is given by $W=\int_{a}^{b} \mathbf{F} \cdot d \mathbf{r}$ '.

Thus, even if you reject Quine's semantic holism and you think that mathematical and logical language is different in kind from empirical language, you need not reject confirmational holism. In order to reject confirmational holism you would need (at the very least) to separate the mathematical vocabulary from the empirical in all of our best scientific theories. Clearly this task is not trivial. ${ }^{23}$ If you still feel some qualms about confirmational holism, though, you may rest assured - this doctrine will be called into question in later chapters when I discuss some of the objections to the indispensability argument. ${ }^{24}$ For the moment, at least, I invite you to join me in accepting confirmational holism.

\subsection{The First Premise Revisited}

Let me close this chapter with a summary of how confirmational holism and Quinean naturalism combine to yield the first premise of argument 2 on page 11. Firstly, you might wonder whether holism is required for the argument. After all, (Quinean) naturalism alone delivers something very close to the crucial first premise. (More specifically $Q O T$ is very suggestive of the required premise.) As a matter of fact I think that the argument can be made to stand without confirmational holism: it's

\footnotetext{
${ }^{23}$ As we shall see, in chapter 4 of this thesis, Hartry Field undertakes this task for reasons not unrelated to those I've aired here.

${ }^{24}$ Most notably, chapters 5 and 6 .
} 
just that it is more secure with holism. The problem is that naturalism is somewhat vague about ontological commitment to the entities of our best scientific theories. It quite clearly rules out entities not in our best scientific theories, but there seems room for dispute about commitment to some of the entities that are in these theories. Holism helps to block such a move since, according to holism, it is the whole theory that is granted empirical support.

So, naturalism tells us to look to our best scientific theories for our ontological commitments. We thus have provisional support for all the entities in these theories and no support for entities not in these theories. For reasons of parsimony, however, we may wish to grant real status to only those entities that are indispensable to these theories. However, we are unable to further pare down our ontological commitments by appealing to some distinction based on empirical support because, according to holism, all the entities in a confirmed theory receive such support. In short, holism blocks the withdrawal of the provisional support supplied by naturalism. And that gives us the first premise of the Quine/Putnam indispensability argument.

In the next chapter I will continue the defence of Quinean naturalism. In particular, I will demonstrate some of the serious problems that face the Eleatic Principle. As we have seen in the present chapter, this principle is the central tenet of Quinean naturalism's main rival: the causal version of naturalism. There are three aspects to this strategy; two are relevant to the task of motivating Quinean naturalism and one that's more general. Firstly, discrediting the Eleatic Principle undermines much of the plausibility of the causal version of naturalism, leaving Quinean naturalism looking all the more attractive. Secondly, I will show that when one considers how the Eleatic Principle might be defended against some of the objections I raise for it, one is drawn towards a position not unlike Quinean naturalism. This again helps fortify the Quinean position. Finally, if the Eleatic Principle were tenable, it would present a quite general problem for mathematical realists, independent of the brand of naturalism subscribed to. Undermining the Eleatic Principle thus clears the way for Platonist philosophies of mathematics. 


\section{Chapter 3}

\section{The Eleatic Principle}

In the previous chapter I identified the crucial difference between Quinean naturalism and Armstrong's causal version of naturalism. The difference, I argued, is that the latter subscribes to the Eleatic Principle, while the former does not. In this chapter I will continue the defence of Quinean naturalism. In particular, I will highlight some problems faced by the Eleatic Principle and suggest that Quinean naturalism is better equipped to deal with these difficulties.

The Eleatic Principle or causal criterion, you will recall, is a causal test that entities must pass in order to gain admission to some philosophers' ontologies. This principle justifies belief in only those entities to which causal power can be attributed, that is, to those entities which can bring about changes in the world. The idea of such a test is rather important in modern ontology, since it is neither without intuitive appeal nor without influential supporters. Its sympathisers (if not supporters) have included David Armstrong [Arm 78, Vol. 2, 5], Brian Ellis [Ell 90, 22], Nancy Cartwright [Cart 83,6] and Hartry Field [Fie 89, 68], to name but a few.

Clearly this principle requires some justification. In this chapter I will look at the arguments that have been put forward for such a principle and suggest some problems for each of these. Of course in such a survey I cannot provide a decisive refutation of the principle (as if anyone ever provides such a thing in metaphysics!), but I do hope to show that, despite its intuitive appeal, the Eleatic Principle's main justifications either look ad hoc or do not justify a version of the principle that delivers the intuitively correct results about some fairly uncontroversial cases. This is not an entirely negative result though. Once we look at the shortcomings of the motivations for the Eleatic Principle a more general principle suggests itself. This more general principle looks very much like Quine's thesis that we are ontologically 
committed to all and only the entities that are indispensable to our current best scientific theories.

Recall that I am not intending to enter into the realist/anti-realist debate in this thesis. I assume realism is true but that some criterion is needed to distinguish real entities from fictional ones. It should also be stressed that any criterion put forward for such a task is a criterion of acceptance. As Keith Campbell puts it:

This search for a criterion for the real must be understood as a search for a criterion for us to count something as real; it will be a principle to apply in determining whether to accord that status, given our current stage of epistemic development. There need not be, and probably cannot be, any critical mark of the real itself; the real is what is, period. [Cam 94, 28]

Also a word or two about a precise formulation of the Eleatic Principle. In [Odd 82] Graham Oddie attacks the Eleatic Principle by systematically questioning a number of specific formulations of it. Although he does this with considerable success, I will not follow him down this path since my criticisms of the Eleatic Principle, for the most part, will not depend on any particular formulation. What I take issue with is the motivation for any formulation of the principle. In any case, it may be useful to specify a particular version, just by way of example. I suggest the following is as good as any (although I stress that nothing hangs on this particular statement of the principle): ${ }^{1}$

Principle 1 (The Eleatic Principle) An entity is to be counted as real if and only if it is capable of participating in causal processes.

In one direction principle 1 is reasonably uncontroversial. Most realists agree that causal activity is a sufficient condition for an entity to be counted as real. ${ }^{2}$ The important question is whether it is a necessary condition. In what follows I will argue that it is not.

In the next five sections I address what I take to be the four best motivations for the Eleatic Principle and I raise difficulties with each in turn. In section $3.1 \mathrm{I}$ discuss an inductive argument sometimes used to justify the causal criterion, while in section 3.2 I look at a very influential epistemic argument put to the same purpose.

\footnotetext{
${ }^{1}$ At this stage I will remain vague about what participation in a causal process amounts to. I will discuss this matter further in section 3.4.

${ }^{2}$ Ian Hacking argues for this claim in [Hac 83] by applying his interventionist test: those entities that can be manipulated as tools in scientific investigations, as opposed to those that are merely tested for, are to be granted real status.
} 
Section 3.3 I devote to an argument which ultimately rests on the claim that only causally active entities can have explanatory power, and this I show to be false. In section $3.4 \mathrm{I}$ address some counter-arguments that sympathisers of the arguments of section 3.3 may appeal to. In the following section I look at the possibility of motivating the causal criterion by rejecting inference to the best explanation while still remaining a realist. In section 3.6 I sum up and suggest what I take to be the moral to be drawn from the difficulties faced by the Eleatic Principle. The moral is not new-it's just that Quinean naturalism looks more promising as a method of demarcating the real from the instrumental and fictional entities-but it is interesting to see how we are led to Quinean naturalism by the shortcomings of the Eleatic Principle. In the brief, final section I recapitulate this and the preceding chapter and outline the chapters ahead.

\subsection{The Inductive Argument}

The first motivation for a causal criterion I will consider is an inductive argument from uncontroversial cases of real entities. We start by noting that there are some fairly widely held intuitions (amongst realists at least) about roughly where the demarcation between the real and the instrumental should be. It should include physical objects, including theoretical entities, perhaps fields and hence waves as disturbances in these fields, amongst the real entities, but should not include (concrete) possible worlds ${ }^{3}$ and frictionless planes. We thus see an initial motivation for the causal criterion: All the things that we intuitively think of as real are the sorts of things that participate in causal processes (in this world at least), whereas those that we intuitively think of as unreal do not participate in such processes.

Thus motivated, the Eleatic Principle is an inductive hypothesis about the way the world is. We look at the things in the world that are uncontroversially real, such as tables and chairs and notice that they are all causally active. Then, by induction, we conclude that all real entities are causally active. At first glance a causal criterion thus motivated looks as if it is purely descriptive-it lacks the normative force that a criterion of acceptance, such as principle 1, ought to have. This defect, however, is easily rectified by appeal to naturalism which does make substantial normative claims about what we ought to believe. For example, if all evidence suggested that all real entities are causally active (a purely descriptive claim), then naturalism

\footnotetext{
${ }^{3}$ Even David Lewis grants that modal realism is counterintuitive [Lew 86b, 135], which is all that I'm claiming here.
} 
commands us to believe that all real entities are causally active (the corresponding normative claim). It is perhaps important to bear in mind that something like naturalism is required to get from purely descriptive theses to normative ones, but in what follows I will assume that such a move can always be made so I will not dwell upon this distinction any longer.

There is a more substantive objection to this motivation though, which is that there are many other properties that the uncontroversially real entities share and that the uncontroversially unreal entities lack. All the uncontroversially real entities are spatio-temporally located, for instance. Indeed, there is argument on this very issue between protagonists of a strictly causal criterion, such as Brian Ellis, and the likes of Hartry Field, who require causal efficacy or spatio-temporal location. The reality of space-time points hangs crucially on whether it's causal efficacy or spatio-temporal location that is the important property. Similarly, we could opt for the property of having a positive rest mass as the mark of the real and again the demarcation would be different.

Worse still, it seems that such an inductive argument is going to depend on what our set of uncontroversially real entities is taken to be. For instance, if we decide to be fairly cautious about selecting the members of this set, and only admit medium sized solid objects, we might conclude that all real entities are coloured! In light of these considerations, how do we come to decide to pin our hopes on causal efficacy and not on other properties?

One possible answer to this question is to argue that it is by virtue of an entity's causal efficacy that we have epistemic access to it, whereas other properties don't force themselves on us like this. ${ }^{4}$ In any case, it seems fairly natural to suppose that we have epistemic access to any entity which we are to count as real. I am thus suggesting that it looks as though it is epistemic access that is in fact the prime motivation for the Eleatic Principle and not the above inductive argument at all. At the very least the above inductive argument needs supplementing and this epistemic argument seems to fit the bill.

\footnotetext{
${ }^{4}$ Recall that we seek "a criterion for us to count something as real" [Cam 94, 28], so we may well admit that there are other properties that all the uncontroversially real entities share, but if we don't (or can't) have epistemic access to these entities by virtue of these other properties, then we can hardly use such properties as criteria of existence.
} 


\subsection{The Epistemic Argument}

The epistemic argument is perhaps the most commonly appealed to motivation for the Eleatic Principle. The argument is simply that even if there were causally idle entities we would have no reason to believe in such entities, since their causal idleness ensures that they don't causally interact with us and, after all, what other way do we have to come to know about entities? ${ }^{5}$

A little consideration, however, reveals that this motivation is also somewhat thin, since the Eleatic Principle, depending on exactly how we formulate it, will either require that entities are causally active, or that they are causally active with humans. The latter alternative, I suggest, looks far too anthropocentric to warrant serious consideration, for surely we ought to believe in stars and planets outside our own light cone ${ }^{6}$ even though they are not causally interactive with us. To deny the existence of such entities is to effectively believe that the earth is the centre of the universe. This leaves only the 'causally active (not necessarily with us)' formulation. On this reading though the epistemological motivation is lost altogether, for there may be many perfectly legitimate real entities involved in causal networks, but because they are not causally interactive with us, they suffer the same epistemic worries as causally idle entities. Again stars, planets and so on outside our light cone are the prime examples here. That is, the set of entities whose existence may be motivated by this epistemic concern form a proper subset of the set of causally active entities.

In fact the Eleatic Principle motivated by epistemic concerns seems to suffer all the same worries that the causal theory of knowledge suffers. ${ }^{7}$ In particular, it seems that we have no reason to believe in future objects (whether causally active or not) and even universal empirical facts obtained by induction are likely to be problematic. Colin Cheyne, who defends an epistemically motivated causal criterion in his paper [Che 98], suggests the rather promising move to kinds of entities in order to overcome some of these problems. He suggests the following principle: ${ }^{8}$

Principle 2 We cannot know that $F$ 's exist unless our belief in their existence is caused by at least one event in which an $F$ participates.

\footnotetext{
${ }^{5}$ This argument is, of course, due to Paul Benacerraf [Ben 73].

${ }^{6}$ That part of the universe close enough to us so that there has been sufficient time since the Big Bang for light to reach us from it.

${ }^{7}$ See [Ste 75] for some of these.

${ }^{8}$ Cheyne offers this principle as a "first attempt" and later in the paper refines it to meet some objections. The objections I have to the principle, though, are not deflected by his later modifications, so I shall be content to deal with his first statement of the principle.
} 
He argues for this principle based mainly on evidence from scientific practicewhat it takes to convince scientists of the existence of a new type of entity. For example, he cites the discovery of the planet Neptune as evidence for principle 2. The existence of Neptune was predicted in 1845-6 jointly by Leverrier and Adams based on Newtonian gravitational theory and anomalies in the orbit of Uranus. It seems that the scientists of the time were unwilling to acknowledge the existence of Neptune until Galle first directly observed the planet in 1846. Cheyne claims that the moral to be drawn from such episodes in the history of science is "interacting is knowing" [Che 98].

Firstly, this example is not entirely appropriate, since we certainly had causal contact with Neptune prior to Galle's visual contact, as Cheyne admits-we had indirect causal contact via its disturbance on the orbit of Uranus. Furthermore, a new planet may not qualify as a new kind of entity since it is of the same kind (namely planet) as the earth. ${ }^{9}$ In any case, leaving these points aside, there is another moral to be drawn from this and the other of Cheyne's examples. The moral I draw is "don't settle for indirect evidence if you can do better". Clearly in the Neptune example direct visual evidence was better than orbital disturbances of Uranus, and scientists sought this better evidence because it was possible. Contrast this with the announcement by Wolszczan and Frail in 1992 of one of the first discoveries of planets outside our own solar system. These planets were detected because of the effects they were having on a nearby pulsar, PSR1257 +12 . Here visual contact was out of the question due to their distance from earth and yet it seems there was no reservation on behalf of the discoverers about making fullblooded existence claims.

[W] e demonstrate that [...] the pulsar is orbited by two or more planet-sized bodies. The planets detected so far have masses of at least $2.8 M_{\oplus}$ and $3.4 M_{\oplus}$, where $M_{\oplus}$ is the mass of the Earth. Their respective distances from the pulsar are $.47 \mathrm{AU}$ and $.36 \mathrm{AU}$, and they move in almost circular orbits with periods of 98.2 and 66.6 days. [Wol 92, 145-147]

Perhaps a better example is that of the discovery of the element germanium. In 1871 there had been no (known) causal contact with this element and, in fact, causal contact wouldn't come until Winckler isolated the metal in 1887. However,

\footnotetext{
${ }^{9}$ This point illustrates a difficulty with the move to kinds of entities - the difficulty of deciding whether a new entity is of a different kind to other entities already accepted. I will not pursue this point though, because I think Cheyne's approach has other, more serious problems.
} 
because of the "gap" in Mendeleeff's periodic table corresponding to the position of germanium, much was known of its chemical behaviour. Cheyne claims that:

If [Mendeleeff] believed, prior to 1887, in the existence of germanium, that belief, although true, would not count as knowledge. It could only be a lucky guess, unless it was actually caused, in an appropriate way, by events in which germanium atoms participated. [Che 98]

Even if I were to agree that if Mendeleeff believed in the existence of germanium prior to 1887 it would not count as knowledge, it seems extremely harsh to call such a belief "a lucky guess". After all, it's not as though he would have had no reason to believe that germanium existed, for there was surely reason to believe that something ought to fill the relevant gap in the periodic table. (Perhaps because of some argument from past predictive success of the table or some appeal to symmetry.) It's not a lucky guess in the same sense that a lottery winner guesses the winning numbers. I claim that, at the very least, Mendeleeff would have had reason to believe in germanium prior to 1887 , which is all that we are interested in for the present purposes. If you accept this claim, then this example, while perhaps not a counterexample to the causal theory of knowledge, looks like a counterexample to an epistemically motivated Eleatic Principle (such as that which principle 2 would motivate) since Mendeleeff had justified belief in a novel substance without the causal contact that principle 2 requires. ${ }^{10}$

It seems clear that appeal to the causal theory of knowledge (which is, after all, what is at the bottom of the epistemic justification) and all its notorious difficulties, is not the right approach for a justification of a causal restriction on ontological commitment. For one thing, an Eleatic Principle thus justified leaves out too many uncontroversially real entities (stars and planets outside our light cone) and secondly, even if one were to accept the causal theory of knowledge, there is no reason to insist that such acceptance implies that causal contact is necessary for justified belief, as the last example illustrates. ${ }^{11}$ Furthermore, Cheyne's move to kinds of entities does not save the Eleatic Principle from these objections.

\footnotetext{
${ }^{10}$ Of course germanium is capable of participating in causal processes, so the Eleatic Principle as I set it out in principle 1 would not rule out belief in germanium prior to 1887 . This serves to highlight the gulf between the Eleatic Principle and an epistemic motivation for it.

${ }^{11}$ It might even be reasonable to argue that many of our basic justified beliefs require causal contact, but this does not mean that inferential beliefs require causal contact. For example, if I am justified in believing $P$ (because I have had causal contact with the truthmaker of $P$, say) and I am justified in believing that $P$ implies $Q$, then surely I am justified in believing $Q$, whether or not I have causal contact with $Q$ 's truthmaker. I am indebted to Peter Forrest and John Bigelow for this point.
} 


\subsection{The Argument from Causal Explanation}

In this and the next section I will address what I take to be the most important argument for the Eleatic Principle. This argument, in its most compelling form at least, is due to David Armstrong. Armstrong has defended the Eleatic Principle in various places. For example, in [Arm 78], he proposes the following dilemma. "Are these [abstract] entities capable of acting upon particulars, or are they not" he asks [Arm 78, Vol. 1, 128]. He then raises difficulties for the first horn of the dilemma, since typically causation involves one change bringing about another, and yet here we have unchanging abstract entities presumably bringing about changes by "some sort of steady, unchanging, pressure" [Arm 78, Vol. 1, 129]. He concludes that "[s] uch a notion is perhaps barely possible, but it is impossible to see how such alleged causal operation could ever be identified" [Arm 78, Vol. 1, 129]. The other horn of the dilemma is simply that "Occam's razor [...] enjoins us not to postulate them" presumably because causally idle entities have no role to play in science [Arm 78, Vol. 1, 130]. As Graham Oddie puts it (on behalf of Armstrong):

They are 'causally idle' and hence 'idle'. Respectable entities work for their living, and there is no social security in Armstrong's universe. [Odd 82, 285$286]$

In [Arm 89] Armstrong has more to say about this second horn of the dilemma.

To postulate entities which lie beyond our world of space and time is, in general, to make a speculative, uncertain, postulation. The postulation may perhaps be defended if it can be presented as explaining some or all of the spatiotemporal phenomena. But if the entities postulated lie beyond our world, and in addition have no causal or nomic connections with it, then the postulation has no explanatory value. Hence (a further step of course) we ought to deny the existence of such entities. [Arm 89, 7-8]

Here Armstrong explicitly cites (and emphasises) the role entities play in explanations as the key to justifying the Eleatic Principle. ${ }^{12}$ In a way this is not at all surprising, since explanation often plays a key role in justifying scientific realism ${ }^{13}$ it would seem only fitting that it should also provide some guide as to the extent of that realism.

\footnotetext{
${ }^{12} \mathrm{He}$ also explicitly appeals to explanation in [Arm 80a, 154-155].

${ }^{13}$ For instance, J.J.C. Smart's cosmic coincidence argument which, very crudely, is that the best explanation for the world behaving as if there were theoretical entities, is that the entities in question actually exist (if not it would be a remarkable coincidence) [Sma 63, 39].
} 
While I agree with Armstrong's rejection of the atypical causal action of the first horn of the dilemma, I don't think that the postulation of causally idle entities has no explanatory value. If this were true then all genuine explanations in science would have to make essential reference to causally active entities. That is, all scientific explanations would be fully causal explanations, but this is not the case. ${ }^{14}$ There are many instances of causally idle entities playing important explanatory roles in scientific theories and I will shortly give a few examples of such cases.

Before I proceed to the examples of explanations in science featuring non-causal entities, I should mention that I do not wish to presuppose any particular model of explanation, ${ }^{15}$ nor any particular model of causation for that matter. For obvious reasons I wish to be as broad-minded as possible about both causation and explanation. I will assume only that an explanation must be enlightening - it must make the phenomena being explained less mysterious. If it does not fulfil this minimal requirement I suggest that it has no right to be called 'an explanation' at all. As for the nature of causation; here I need only appeal to some widely held intuitions about what sorts of entities are taken to be causally idle (for example, geometric entities and functions). Beyond this, I leave the nature of causation completely open.

I should also explain why I won't be appealing to a couple of obvious examples of non-causal explanations. The first is mathematical explanations of mathematical facts. For instance, when seeking an explanation of Gauss's Theorema Egregium ${ }^{16}$ one does not find a causal one. Perhaps this goes without saying. Anyway, I will not be discussing such examples because, although they are undoubtedly noncausal explanations, they are not explanations of events, and it is for these that it is sometimes claimed that no non-causal explanations may be found. ${ }^{17}$

The second example of non-causal explanations which I won't be considering are the Einstein-Podolsky-Rosen cases in quantum mechanics. It might seem a little odd to leave these aside, since many would argue that these offer some of the best candidates for non-causal explanations. My reason for leaving these cases aside,

\footnotetext{
${ }^{14}$ In the discussion following a presentation of the material from this chapter, Armstrong denied that his argument rested on the claim that all scientific explanations are causal explanations. This claim is hard to reconcile with the textual evidence I cite above, but whomever the argument from causal explanation ought to be attributed to, it is an interesting argument with considerable plausibility and deserves serious attention.

${ }^{15}$ Although I am inclined towards Philip Kitcher's account of explanation as unification. See [Kit 81] for details.

${ }^{16}$ This remarkable theorem states that the Gaussian curvature of a surface is intrinsic [McC 94, 148].

${ }^{17}$ David Lewis, for one, claims that there are no non-causal explanations of (particular) events [Lew 86a, 221].
} 
however, is that I think they are very difficult to interpret. It is not clear to me whether the explanations are non-causal, or as some suggest, reverse-causal. ${ }^{18}$ Such cases cast significant doubt on the notion of causal explanations in which causation is taken to be time asymmetric. The examples I have chosen, however, are more damaging to the causal theory generally.

\subsubsection{The Bending of Light}

We are told that the path of a beam of light is bent in the vicinity of a massive object; the more massive the object, the greater the bending. This result was first observed in 1919 by comparing the position of a star when its light passed near the sun (during a solar eclipse) with its usual position. What is the explanation for this bending?

The preferred explanation, offered by general relativity, is geometric. It's not that something causes the light to deviate from its usual path; it's simply that light travels along space-time geodesics ${ }^{19}$ and that the curvature of space-time is greater around massive objects. Typically the defender of causal explanations will point out that it is the mass of the object that causes the curvature of space-time, and so there is an underlying causal explanation after all. There are two fairly serious problems with this reply though. The first is the difficulty of spelling out, in a causally acceptably way, how it is that mass brings about the curvature of spacetime. After all, it can't be that there is an exchange of energy and/or momentum between the object and space-time, as some accounts of causation require. As I have already said, I wish to remain non-committal with respect to the details of causation, but it seems that any account that permits mass to cause the curvature of space-time is unintuitive to say the least.

Note that I acknowledge that there is undoubtedly covariance between mass and curvature, but I deny that all covariance need be cashed out in terms of causation. ${ }^{20}$ For example, the angle sum of a triangle covaries with the shape of the space in which it is embedded, but one is not inclined to say that the angle sum of a triangle causes the shape of the relevant space. It seems to me that the case of mass and the shape of space-time is similar to this. Another way of looking at this difficulty is by asking the question: Why is it not the case that the curvature of space-time causes the

\footnotetext{
${ }^{18}$ See, for example, [Pric 96, Pric 98] for interesting discussions of what amounts to a reversecausal account of the Einstein-Podolsky-Rosen cases.

${ }^{19}$ In fact it's probably better to say (tenselessly) that light lies along space-time geodesics.

${ }^{20}$ Physicists, I'm told, are more inclined to think of curvature as a manifestation of mass.
} 
mass? Simple covariance doesn't guarantee that one of the factors causes the other.

The second problem for this line of argument is that it seems there are solutions to the Einstein equation for empty space-times in which the curvature of space-time is not identically zero. These are the non-Minkowski vacuum solutions [Pea 92, 17]. ${ }^{21}$ Thus we see that, at the very least, mass cannot be the only cause of curvature. What then is causing the curvature in the vacuum solutions case? There is nothing to cause it! It simply looks as though the curvature is uncaused. Why then insist on a causal explanation of the curvature in the universes with mass? I suggest that there is no reason at all, and that we ought to simply accept the geometric explanation for the bending of light.

\subsubsection{Stellar Stability}

A star such as our sun is subject to tremendous gravitational forces, which drive all the matter of the star inwards towards the centre. What prevents a star from collapsing further? There are four cases to be considered when answering this question.

The first case is where a star is said to be in hydrostatic equilibrium. Here the gravitational force is trying to compress the star and this force is balanced by pressure from within the star. Most of this pressure is provided by the pressure of the gases themselves, although in very luminous stars radiation pressure is also a factor [Abe 87, 507]. Here the explanation is clearly causal.

The next three cases are concerned with when the nuclear fuel ${ }^{22}$ is largely used up and hydrostatic equilibrium is upset. Case two is where the star has a mass of about the same as our sun (written as $1 \mathrm{M}_{\odot}$ ). Here, after another nuclear fusion reaction (the triple-alpha reaction) whose details need not concern us, the gravitational forces take over and the star collapses down to another stable arrangement, the so-called white dwarf. The reason that a white dwarf does not collapse further is completely

\footnotetext{
${ }^{21}$ The situation is somewhat complicated though, since the positive mass theorem of general relativity states, in effect, that such solutions must have a singularity, without which the spacetime would be flat. This is assuming the ADM (after R. Arnowitt, S. Deser and C.W. Misner) conception of mass. (This is a global conception of mass.) Adopting the stress-energy tensor conception of mass (which is a local conception of mass), however, non-singular, non-Minkowski, vacuum solutions are possible. For example, the analytic extension of the Schwarzchild metric (see [DIn 92, 219-221]) through the singularity has non-zero ADM mass but the stress-energy mass is everywhere zero. What is more, this space-time is non-singular and non-flat. I am indebted to Robert Bartnik and Matthew Spillane for their help with this point.

${ }^{22}$ The "nuclear fuel" is hydrogen, which is converted to helium via the proton-proton chain, releasing large amounts of energy in the form of electromagnetic radiation.
} 
different from that in the previous case. The star cannot collapse further because of electron degeneracy. ${ }^{23}$ Here there is no internal force counteracting gravity, and thus, it seems, no causal explanation.

The next two cases are similar to the second, except that the mass of the star in these cases is greater than the Chandrasekar mass limit of about $1.4 \mathrm{M} \odot$ and so the electron degeneracy can be broken. The result depends on how much greater than $1.4 \mathrm{M}_{\odot}$ the mass happens to be. If it is somewhere between $1.4 \mathrm{M}_{\odot}$ and $2.0 \mathrm{M}_{\odot}$, the star is again prevented from further collapse by the Pauli exclusion principle; this time by neutron degeneracy. ${ }^{24}$ The resultant star is called a neutron star since it consists largely of neutrons. Again the explanation for the collapse stopping seems non-causal. If, however, the mass of the star is greater than about $3 \mathrm{M}_{\odot}$, then neutron degeneracy is also broken and it continues to collapse to a black hole.

David Lewis considers this example, albeit in less detail, and concludes that in the cases where the collapse stops because of the Pauli exclusion principle:

The state-space of physical possibilities gave out. (If ordinary space had boundaries, a similar example could be given in which ordinary space gives out and something stops at the edge.)

[I]nformation about the causal history of the stopping has been provided, but it was information of an unexpectedly negative sort. It was the information that the stopping had no causes at all, except for all the causes of the collapse which was a precondition of the stopping. Negative information is still information. If you request information about arctic penguins, the best information I can give you is that there aren't any. [Lew 86a, 222-223]

This reply seems rather odd though. The oddness stems from the conjunction of the assertion "the stopping had no causes at all" and the claim that this is a causal explanation. ${ }^{25}$ There is only one way to make sense of this, and that is if Lewis really does see this case as analogous to that of ordinary space giving out. This analogy, however, seems entirely inappropriate since, as we have seen, the Pauli exclusion principle prevents stars of certain masses from collapsing further; it does

\footnotetext{
${ }^{23}$ The Pauli exclusion principle states that no two particles of the same spin can occupy the same energy state at the same time. A system in its lowest possible energy state thus has every energy level, from the lowest upwards, occupied by just one particle. Such a system is said to be degenerate and can collapse no further.

${ }^{24}$ The gravitational collapse is sufficient to force electrons to combine with protons to form neutrons, in what is essentially the reverse of radioactive $\beta$-decay.

${ }^{25}$ For David Lewis a causal explanation is "the provision of information about causal histories" [Lew 86a, 221].
} 
not claim that it is not possible to obtain a more collapsed state. Presumably if the white dwarf had a greater mass at the crucial second red giant stage its collapse would have continued.

The case seems more analogous to a person trying and failing to break a door open by charging it with their body. It is not that physical space has given out; it is just that the person's momentum isn't great enough. In the latter case a causal story of why the door could not be broken open can be provided in terms of the door providing a resisting force, and it is precisely the lack of such a story in cases two and three of the stellar collapse that makes them cases of non-causal explanation. In effect I am denying that Lewis's causal story is a satisfactory explanation of the phenomenon, since it fails to give an account of what prevents some stars and not others, from collapsing down to more compact configurations. The non-causal explanation (i.e. appealing to the Pauli exclusion principle) has no such shortcoming. ${ }^{26}$

\subsubsection{Antipodal Weather Patterns}

We discover that at some time $t_{0}$ there are two antipodal points $p_{1}$ and $p_{2}$ on the earth's surface with exactly the same temperature and barometric pressure. What is the explanation for this coincidence?

Notice that there are really two coincidences to be explained here: (i) Why are there any such antipodal points? and (ii) Why $p_{1}$ and $p_{2}$ in particular? The first explanation I will offer is a causal explanation (i.e. featuring only causally active entities) and it addresses the second question. This explanation, presumably, will trace the causal history of the current weather patterns, to arbitrary fine detail if necessary, to account for the weather patterns at $p_{1}$ and $p_{2}$. In particular, the temperature and pressure readings at $p_{1}$ and $p_{2}$ at time $t_{0}$ will be accounted for. Notice that an explanation such as this does not explain why $p_{1}$ and $p_{2}$ have the same temperature and barometric pressure, just why each has the particular temperature and pressure that they have, and that these happen to be the same. Thus an important part of the original phenomenon is left unexplained.

This case looks similar to that of explaining why there were 11 fatalities on New South Wales roads over the 1995 Easter break. The causal story will give the causal history of each fatality but will not explain why, in particular, there were

\footnotetext{
${ }^{26}$ The explanation in terms of the Pauli exclusion principle may yet turn out to be causal if the principle is explained (causally) by a quantum-hidden variable theory. However no such theory is forthcoming at the present time.
} 
11 fatalities. This does not seem like such a deficiency in the road fatalities case, since it seems as though there is nothing (significant) left to be explained above and beyond what the causal story tells us. The case of the antipodal weather conditions though is entirely different.

The difference is due to a theorem of algebraic topology which states that for any time $t$ there are antipodal points on the surface of the earth which simultaneously have the same temperature and barometric pressure. ${ }^{27}$ This theorem, or more correctly the proof of this theorem, provides the missing part of the causal explanation. It guarantees that there will be two such antipodal points at any time and, furthermore, the explanation makes explicit appeal to non-causal entities such as continuous functions and spheres.

Notice, though, that this explanation also has its limitations - it does not explain why it is $p_{1}$ and $p_{2}$ in particular that have the same temperature and pressure. So we see that for a complete explanation of the phenomenon in this example, we require both causal and non-causal elements in the explanation.

\subsubsection{The FitzGerald-Lorentz Contraction}

The special theory of relativity tells us, amongst other things, that a body in motion, relative to some inertial reference frame $\mathcal{F}$, suffers a FitzGerald-Lorentz contraction. This is a reduction in the length of the body in the direction of motion, as measured by an observer stationary with respect to $\mathcal{F}$. What is the explanation for this contraction?

Minkowski's great contribution to relativity was in offering an elegant explanation for the Lorentz transformations (including the FitzGerald-Lorentz contraction). This explanation appeals to the now familiar concept of space-time, that is, a threeplus-one-dimensional manifold, which consists of three spatial dimensions and one temporal. Minkowski realised that one of the key assumptions of special relativity, the constancy of the speed of light, could be formalised as the satisfaction of the equation:

$$
\left(\Delta x_{1}\right)^{2}+\left(\Delta x_{2}\right)^{2}+\left(\Delta x_{3}\right)^{2}-c^{2}(\Delta t)^{2}=0
$$

in any inertial frame. Here $x_{1}, x_{2}$ and $x_{3}$ are the spatial coordinates, $t$ is the temporal coordinate and $c$ is a constant (the speed of light in a vacuum). Minkowski then

\footnotetext{
${ }^{27}$ This theorem is a corollary of the Borsuk-Ulam theorem, combined with some minor structural assumptions (i.e. that the earth is approximately spherical in shape and that temperature and pressure change continuously across its surface) [Kos 80, 157-159].
} 
introduces the imaginary time coordinate

$$
x_{4}=i c t
$$

where as usual $i=\sqrt{-1}$. So (3.1) becomes:

$$
\left(\Delta x_{1}\right)^{2}+\left(\Delta x_{2}\right)^{2}+\left(\Delta x_{3}\right)^{2}+\left(\Delta x_{4}\right)^{2}=0
$$

and (3.2) will be satisfied in every inertial frame if the quantity

$$
\sigma^{2}=\left(\Delta x_{1}\right)^{2}+\left(\Delta x_{2}\right)^{2}+\left(\Delta x_{3}\right)^{2}+\left(\Delta x_{4}\right)^{2}
$$

is invariant under Lorentz transformation. This, says Einstein, "shows that the Lorentz transformation so defined is identical with the translational and rotational transformations of Euclidean geometry, if we disregard the number of dimensions and the relations of reality" [Ein 67,31]. That is, the FitzGerald-Lorentz contraction is nothing more mysterious than the apparent shortening of an object in one dimension when a new set of axes are chosen, inclined at some angle to the old. This latter thesis is the invariance of length under translation and rotation and is expressed mathematically as the invariance of the quantity

$$
s^{2}=\left(\Delta x_{1}\right)^{2}+\left(\Delta x_{2}\right)^{2}+\left(\Delta x_{3}\right)^{2}
$$

under linear transformations with determinate $|1|$ (i.e. the transformations are neither contractions nor expansions).

The explanation for the FitzGerald-Lorentz contraction is seen very clearly when one realises that the quantity $s^{2}$ in equation (3.4) is not invariant under Lorentz transformation in Minkowski space (although it is under rotation and translation in $\mathbb{R}^{3}$, as we have seen). The relevant invariant in Minkowski space is $\sigma^{2}$, as given by equation (3.3). I also stress the obvious here, that this is a purely geometric explanation featuring such non-causal entities as the Minkowski metric and other geometric properties of Minkowski space. ${ }^{28}$

\subsection{Causal Relevance}

In this section I investigate another reply that supporters of the causal criterion are liable to make. This is to deny the causal idleness of the entities in examples such

\footnotetext{
${ }^{28} \mathrm{I}$ am indebted to Jack Smart for drawing my attention to this example in both discussion and in his paper [Sma 90].
} 
as those presented in the last section. One plausible way which this can be done is to claim that the entities in the explanation are causally relevant but not causally efficacious. Frank Jackson and Philip Pettit give a good account of this approach in [Jac 90]. Although Jackson and Pettit don't specifically put the notion of causal relevance to work salvaging the causal criterion, nonetheless, their program could be used for this purpose.

Consider a case of trying to fit a square peg of side length $\ell$ into a round hole of diameter $\ell$. Clearly it will not go. The first reason is non-causal: because of the squareness of the peg (and the roundness of the hole). The second is causal: the resistance offered by the overlapping portion of the peg. Furthermore, it seems that someone in possession of the squareness explanation knows more than someone who knows only the overlapping explanation. Jackson and Pettit suggest, and I agree with them here, that although the abstract property of squareness did not cause the overlapping, nor did it combine with the overlapping to produce the blocking, it is certainly true that the squareness was efficacious only if the overlapping was. They conclude that the abstract property of squareness is not causally efficacious (at least in this example). There is a sense, though, in which it is not causally irrelevant either. It is not irrelevant in the way in which, say, the colour of the peg is. On this, Jackson and Pettit have the following to say:

Although not efficacious itself, the abstract property was such that its realization ensured that there was an efficacious property in the offing. [Jac 90, 116]

That is, the property of squareness programs for the efficacious property of overlapping portions.

While I think there is much to be said for the causal relevance approach, in the end it won't save the causal criterion, for as I see it there are two serious difficulties facing this approach. Firstly, I don't think that this defence will work for all explanations making use of non-causal entities. It will work only for those in which a fully causal explanation (i.e. one in which all the entities in question are causally efficacious) is on offer as well as the non-causal one, or where there are noncausal elements in a largely causal explanation. Thus, this strategy won't work for the FitzGerald-Lorentz contraction case, for instance, where only one explanation is on offer and it is non-causal, in that it makes no appeal to causally active entities (only to the geometric properties of Minkowski space). Secondly, although it enables supporters of the causal criterion to classify many apparently non-causal entities as 
causal, this is done at a fairly high price: significant blurring of the distinction between the causal and the non-causal. This blurring, if serious enough, is just the sort of thing that antagonists of the causal criterion would welcome. After all, if the property of squareness can enter into causal explanations, albeit in a subsidiary role (i.e. as causally relevant rather than causally efficacious), it seems that the causal requirement lets in too much. I am more inclined to admit that causally idle entities can have explanatory power than to fiddle with the definition of 'causal' in this way.

One final move is left open to the supporter of the claim that only causally active entities can have explanatory power, and this is to argue that the geometry of spacetime, for instance, while not being causally efficacious, nor programming for causally efficacious properties, may predetermine the range of possibilities. Space-time is thus seen as a structuring cause in Dretske's language [Cam 94, 36]. This move will allow the supporter of the causal criterion to classify the remaining recalcitrant explanations I've presented as fully causal explanations. But now I think that the difference between such a position and my own is entirely terminological. After all, what is the difference between allowing that there are causally idle entities with explanatory power, on the one hand, and, on the other, maintaining that only causally active entities have explanatory power but that some of those entities might be structures that are not directly involved in causal chains? I suggest that whether one classifies such structures as causal or not, the important point is the recognition of the importance of such structures in scientific explanations.

Where does this leave us then? Either there are causally idle entities with explanatory power, such as the geometry of space-time, or only causally active entities have explanatory power but they may include structural elements such as the geometry of space-time and programming properties such as continuous temperature distribution functions. Clearly it is the former conclusion I have been arguing for, but the latter will do as well. If the causal criterion is motivated by a notion of causally active entity which must include geometric properties, continuous functions and the like, then it is ill equipped to make the demarcation required of it. Geometric properties and mathematical entities are just the sorts of entities the causal criterion is usually appealed to to eliminate. Perhaps this is not a terribly damaging argument against the causal criterion. After all, you could just bite the bullet and accept that the causal criterion does not rule out mathematical and geometric properties as is commonly thought. I have no disagreement with a causal criterion thus construed except that I fail to see what the word 'causal' is doing in its name, since surely such entities are the paradigm cases of non-causal entities. It looks as though the 
causal criterion is preserved in name only.

\subsection{Rejection of Inference to the Best Explanation}

In the previous two sections I discussed, at some length, the motivation for the Eleatic Principle that rested ultimately on the claim that only causally active entities can have explanatory power. It might be useful at this stage to make explicit one other assumption of that argument. This assumption is that we have ontological commitment to the entities in our best scientific explanations, which is simply to say that inference to the best explanation is a justified form of inference-it's just that supporters of the argument of section 3.3 are committed to all explanation featuring only causally active entities. Once the argument is put that way, another defence of the Eleatic Principle presents itself. This defence is to accept that there are non-causal entities with explanatory power, but to reject inference to the best explanation in its most general form. This position has been defended by Nancy Cartwright in her book How the Laws of Physics Lie [Cart 83] in which she argues for inference to the most likely cause instead of the more general inference to the best explanation. Brian Ellis argues for a similar position in Truth and Objectivity [Ell 90] in which he accepts that science makes extensive use of non-causal explanation but that only fully causal explanations carry ontological commitment. ${ }^{29}$ If some restriction on inference to the best explanation to causal explanations can be sustained then the Eleatic Principle is justified trivially. I consider Cartwright's and Ellis's arguments for such a restriction in this section.

\subsubsection{Ellis's Argument}

Ellis is a scientific realist and, like many other Australian realists, is so largely because of Smart's cosmic coincidence argument (which I mentioned in section 3.3). There is one difference though. Ellis does not accept inference to the best explanation as Smart does. Ellis claims that "[o]ntological commitment can derive only from causal process explanations" [Ell 90, 22]. The latter is enough for a restricted version of Smart's argument for scientific realism to go through. The resulting realism is restricted to causally active entities.

\footnotetext{
${ }^{29}$ Of course there are other issues on which Cartwright and Ellis have substantial disagreement. For instance, Cartwright is anti-realist about scientific theories whereas Ellis is not.
} 
The ontology does not admit abstract entities like propositions and sets, unless these can somehow be reduced to entities of other kinds. For such entities have no causes or effects, have no location in space or time, and cannot influence any causal processes. It is argued that while such entities may have a role in model theoretic explanations, acceptance of such explanations carries no ontological commitments; only the acceptance of causal explanations carries any such commitment to the entities involved. The entities occurring in our model theories should generally be regarded as fictions. [Ell 90, 5]

His reason for restricting inference to the best explanation in this way is apparent once we distinguish between two quite different types of scientific explanation. The first is the causal explanation which, on Ellis's account of causation, will typically involve a story about exchanges of energy between physical entities. The second type of explanation is the more general model theoretic explanations, which typically idealise away from real situations. These latter explanations are used as backgrounds for causal explanations. For example, Newton's first law provides the background for a causal explanation of why some moving object comes to rest. These model theoretic explanations typically feature such obviously fictional entities as frictionless planes, non-turbulent, laminar flow and inertial reference frames, so we should not accord real existence to the entities that feature in such explanations.

While I agree that this argument presents good reason to be suspicious of entities in such model theoretic explanations, it says nothing of abstract entities that feature in causal explanations. For example, in a fully causal account of a billiard ball collision (i.e. with frictional forces etc.) we will find reference to vectors. ${ }^{30}$ Ellis acknowledges as much in the following passage.

The main argument for realism about theoretical entities is also, apparently, an argument for the existence of forces, fields, numbers, sets, spatio-temporal relationships, possible worlds, and many other kinds of things. [Ell 90, 60-61]

But he has another reason for insisting that abstract entities have no real existence.

The basic reason for resisting abstract particulars is that the world we can know about would be the same whether or not they existed. [Ell 90, 79]

\footnotetext{
${ }^{30}$ Recall that it is this feature of mathematics that Putnam so forcefully argued for in his realist days. He argued that mathematical entities feature indispensably in the very same explanations that lead realists to believe in theoretical entities. See [Put 71] and page 13 section 1.2 .2 of this thesis.
} 
The key phrase here is 'the world we can know about'. Clearly some causal theory of knowledge is alluded to here, for otherwise the statement is patently false. ${ }^{31}$ So in the end Ellis's restriction of the application of inference to the best explanation to causal process explanations will not provide a justification for the Eleatic Principle (by his own admission-see second last quote), so we are back to the epistemic justification which I discussed and dismissed in section 3.2.

\subsubsection{Cartwright's Argument}

Nancy Cartwright is also a realist of sorts. She is a realist about theoretical entities but not about scientific theories. She sums up her view rather nicely in the following passage.

I believe in theoretical entities. But not in theoretical laws. Often when I have tried to explain my views on theoretical laws, I have met with a standard realist response: 'How could a law explain if it weren't true?' Van Fraassen and Duhem teach us to retort, 'How could it explain if it were true?' What is it about explanation that guarantees truth? I think there is no plausible answer to this question when one law explains another. But when we reason about theoretical entities the situation is different. The reasoning is causal, and to accept the explanation is to admit the cause. [Cart 83, 99]

In her rejection of inference to the best explanation she aligns herself more with anti-realists such as Bas van Fraassen ${ }^{32}$ but accepts theoretical entities that feature in causal explanations for the same sorts of reasons as Ian Hacking. ${ }^{33}$ So whereas Ellis is a realist who rejects inference to the best explanation in its most general sense, it's perhaps more appropriate to see Cartwright as an anti-realist who accepts inference to the most likely cause. Hacking's arguments ensure that causal activity is a sufficient condition for ontological commitment, whereas general anti-realist considerations ensure that it is also a necessary condition. Once put this way it is clear, I think, that for me to reply to Cartwright would involve entering into the realist/anti-realist debate, which I said at the outset was too large a task to tackle in this thesis. Nevertheless, I feel obliged to say something in reply to Cartwright, but before I do this I need to clear up an ambiguity in Cartwright's position.

\footnotetext{
${ }^{31}$ Elsewhere Ellis is more explicit about his endorsement of a causal theory of knowledge [Ell 90, $7]$

${ }^{32}$ See [vanF 80] for details of van Fraassen's rejection of inference to the best explanation.

${ }^{33} \mathrm{See}$ footnote 2 in this chapter or [Hac 83] for further details.
} 
Recall that Cartwright admits theoretical entities that are causes of some phenomena which require explaining. The ambiguity revolves around what constitutes an event (or phenomenon) in need of explanation. For example, suppose that all entities that are causally active are the cause of some event or other. Then Cartwright's inference to the most likely cause may warrant belief in these entities as the most likely causes of their respective events. Notice that nothing here ensures that the entities in question are causally active with us. On the other hand, one could argue that what she takes to be an event in need of explanation must be an event which we know about. That seems uncontroversial enough, but now depending on how we spell out the 'we know about' claim, it looks as though Cartwright cannot admit causally active entities which are not causally active with us.

As it turns out I think that it is the latter position which Cartwright intends. This can be best seen by considering a case where there is some event, $e$, which we have no causal contact with and asking the question: what reason does Cartwright have to believe that such an event occurred? One way would be if we directly observe $e$ or observe a result of $e$, but this would mean that we have causal contact with $e$, which is ruled out by construction. The important question is whether, for Cartwright, there can be any other way of knowing about $e$. It seems not, given what she says about inference to the best explanation. Recall that inference to the best explanation is not an admissible inference for Cartwright, so it can't be that $e$ explains some other event or phenomena, unless of course $e$ is the cause of that event or phenomena, and this is also ruled out by construction. What other reason can we have to postulate a causally isolated entity on Cartwright's account? I can think of none, so I must conclude that Cartwright is indeed committed to only those entities which are causally active with us. I will now use this reading of Cartwright's position on some examples I have considered previously. These examples will show how Cartwright's position seems unable to give the intuitively correct result in what are fairly uncontroversial cases.

The first example is that of the stars and planets outside of our light cone. These are theoretical entities that are not the cause of anything (that we can observe), so it seems on Cartwright's account they ought not be granted "real" status. As I've mentioned previously, in relation to the epistemically motivated Eleatic Principle (cf. section 3.2), this seems like the wrong answer. Note, however, that I am not claiming that Cartwright is committed to this view, just that to avoid this conclusion will require some additional argument. In the absence of such an argument the undesirable conclusion does seem to follow from inference to the most likely cause 
alone.

The other example I'd like to reconsider is the case of belief in the existence of germanium prior to 1887 . Recall that I assume that there had been no known causal contact with germanium until 1887, but that it was postulated in 1871 on the basis of a "gap" in Mendeleeff's periodic table. It seems Cartwright must deny any good reason to believe in germanium at that time since its causal isolation guarantees that it couldn't have been the cause of anything (that we knew about) and the only appropriate inference she allows is inference to the most likely cause. Again I trust that your intuitions suggest that this is the wrong answer!

Much more could be said about Cartwright's ontology and, in particular, her inference to the most likely cause, but in the end I find examples such as those above give us good reason to suspect that her project will not work as a defence of a causal constraint on existence claims.

\subsection{The Moral}

In this section I wish to show that there is a positive message to be gleaned from what looks like the complete lack of support for the Eleatic Principle. In section 3.1 we saw how the inductive argument for the principle is in itself inadequate, but leads rather naturally to epistemic considerations. These, in turn, suffer from the same sorts of objections as the causal theory of knowledge (which is, after all, what underlies the epistemic motivation). In particular, we saw how this motivation yields some undesirable consequences in some fairly straightforward cases; for instance, that we ought not believe in stars and planets outside of our own light cone. Such a position is surely unpalatable to even the staunchest defenders of the principle. ${ }^{34}$ In section 3.3 I examined what I take to be the most important argument for the Eleatic Principle, due to David Armstrong. Armstrong claims that causally idle entities have no explanatory value and hence should not be considered to be real. This argument was seen to rely on the assumption that only causally active entities

\footnotetext{
${ }^{34}$ It may well be possible to arrive at a formulation of the principle that overcomes many, if not all, of the objections that I discuss in this chapter. Keith Campbell, another defender of a causal test, addresses this issue in his paper [Cam 94] and concludes that this is indeed possible. It seems to me, however, that a criterion so motivated is left looking ad hoc in the extreme. Its only motivation is that it ought to give the right answers to all the problem cases and still look something like a causal test. So whereas Campbell and I agree that the criterion must be framed in such a way that items lying outside our light cone are not excluded, I do not agree with him that "such adjustments do not call the causal test into serious question" [Cam 94, 32]. Such adjustments deprive it of any reasonable motivation.
} 
have explanatory power, and this was show to be a mistake. Finally, in section 3.5 we saw that motivating the Eleatic Principle by appeal to a restriction of inference to the best explanation to causal explanation also faces serious problems.

The moral to be drawn from all this is best illustrated by considering the deficiencies of the arguments for the Eleatic Principle and how they might be overcome. For instance, the epistemic justification failed because it ruled out entities such as germanium prior to 1887 and stars and planets outside our light cone, which seem reasonable to believe in. There is a way of getting around these problem cases though, and that is to appeal to some sort of "rounding out" principle. Thus, a causalist may argue that even though an epistemically motivated Eleatic Principle rules out stars and planets outside our light cone, nevertheless, such entities may be included on the basis of this "rounding out" principle. ${ }^{35}$ But now one wonders what the purpose of the Eleatic Principle is, if it is so easily overruled when it gives the wrong answer. Why not try to be more explicit about what the "rounding out" principle amounts to and just appeal to it in the first place?

It seems this rounding out principle, if it allows belief in stars and planets outside our own light cone, is going to do so for reasons of symmetry or, more generally, for reasons of theoretic virtue. That is, the astronomical theory that posits stars and planets outside our own light cone is a better theory than its counterpart that does not posit such entities. (For one thing, the latter theory is stuck with the problem of explaining why the earth is apparently at the centre of the universe.) Now notice that most entities which are causally active are likely to be needed in (causal) explanations of certain phenomena. ${ }^{36}$ Thus, using this "rounding out" principle, at least as I've outlined it here, will effectively subsume one direction of the Eleatic Principle causally active entities will gain admission to our ontology because they are needed for our scientific theories. This principle looks more promising than the Eleatic Principle, since it seems to avoid the more obvious pitfalls of the epistemically motivated Eleatic Principle and yet, because in many cases both principles yield the same results, it retains much of the Eleatic Principle's intuitive appeal. In effect, I'm suggesting that the Eleatic Principle may be a good ontological "rule of thumb" but may not be the final arbiter on such matters. ${ }^{37}$

\footnotetext{
${ }^{35}$ For instance, in conversation Alan Musgrave has suggested this.

${ }^{36}$ For example, the stars and planets inside our own light cone will be needed to explain why we see points of light when we look up at the night sky.

${ }^{37}$ Indeed, there is some evidence to suggest that even some of the Principle's supporters may agree with me here. For instance, in the following passage David Armstrong suggests that the causal criterion is a heuristic device for isolating those entities for which it is hoped that a reductive
} 
Alternatively, let's cast our minds back to the Armstrong argument for the Eleatic Principle. This argument placed the responsibility for questions of ontology firmly upon the explanatory power of the entities in the theories in which they occur. My point of disagreement was that acausal entities also have explanatory power, so we should not confine our attention to causally active entities. While I agree that explanations featuring causally active entities are often very good explanations, and perhaps we ought to seek such explanations when appropriate, it's just pointless to restrict our attention to these explanations, since they do not exhaust the acceptable explanations of science. There is, however, an important insight in Armstrong's argument. This insight is seen in the move to using explanatory power as a guide to deciding questions of ontology. If we heed this advice, but do not restrict the acceptable explanations of science to those featuring causally active entities, we find ourselves once again with a very general principle for deciding ontology, not unlike the "rounding out" principle we arrived at in the last paragraph. In both cases the principle places the responsibility for ontology on theoretic virtue (symmetry in the former case and explanatory power in the latter) and in both cases the principle goes well beyond the scope of the Eleatic Principle. ${ }^{38}$

Thus this more general principle now begins to look very much like a thesis that Quine has long advocated. We've already seen how Quinean naturalism counsels us to draw our ontological commitments from our best theories. More specifically: We are ontologically committed to all and only the entities that are indispensable to our current best scientific theories. I stress that this is not intended as a rigorous argument for Quinean naturalism, for I'm sure there are other ways to avoid the difficulties faced by the Eleatic Principle; however, the fact that we have arrived at a very similar position by considering these difficulties, and making rather natural responses to them, does suggest that the Quinean thesis is better equipped to embark on the difficult task of deciding which entities we allow entry to our ontology.

analysis may be given.

The argument from lack of causal power is simply intended as a reason for thinking that the research programme [of giving an account of the truth conditions of numbers, classes, propositions etc. in terms of particulars and their properties and relations] is a promising one. [Arm 78, Vol. 2, 5]

In other words, he suspects that causally idle entities are dispensable to science but admits that the real work is the required reduction of such entities to more respectable entities.

${ }^{38}$ For both these principles it will be an open question whether (abstract) mathematical entities will be granted real status, whereas the Eleatic Principle rules them out a priori. Indeed, this may count as another point in favour of the more general principle. Any criterion which claims to settle such long standing problems as the ontological status of mathematical entities in such a straightforward manner ought to be treated with some suspicion. 


\subsection{Recapitulation}

So far I have discussed the Quine/Putnam version of the indispensability argument and shown how it depends on the Quinean doctrines of naturalism and holism. I then defended the Quinean reading of naturalism against causal versions of naturalism. The latter explicitly or implicitly appeal to a causal test in order to decide which entities are legitimately deserving of real status, with only the causally active entities able to pass this test. In the present chapter I argued against the use of such a test. It is important to see the problems associated with such a test for at least two reasons. Firstly, assuming that it is desirable to hold some version of naturalism, discrediting the causal version raises the stocks in its main rival: Quinean naturalism. The second reason is that in considering the problems with the Eleatic Principle and how they might be rectified, we found that one is drawn in a very natural way to Quinean indispensability theory. None of this counts as a positive argument for the Quinean position; indeed, as is often the case with such basic doctrinal beliefs, it is difficult to produce persuasive positive arguments. The best one can hope for is that the doctrine in question fare well in cost-benefit comparisons with the main contenders. I hope you agree that the preceding chapters have shown that the Quinean position, if not the best approach to matters of ontology, is at least highly plausible.

Much more could be said concerning the Quinean backdrop, particularly with regard to naturalism, but instead I shall move on to consider some explicit attacks on the indispensability argument. I n discussing these attacks, however, issues concerning the Quinean backdrop will arise again. This will hopefully bring into sharper focus the doctrines of naturalism and holism and underline their importance to the whole debate. I will devote most of the remainder of this thesis to defending the Quine/Putnam version of the indispensability argument. 



\section{Chapter 4}

\section{Field's Fictionalism}

The indispensability argument has suffered attacks from seemingly all directions in recent times. First Charles Chihara [Chi 73] then Hartry Field [Fie 80] raised doubts about the indispensability of mathematics to science, and more recently Elliot Sober [Sob 93] and Penelope Maddy [Mad 92, Mad 95a, Mad 95b] have raised separate concerns as to whether we really ought to be committed to entities which are indispensable to our best scientific theories. In the next three chapters I will address some of these objections and I'll show that the indispensability argument can survive these attacks.

I begin in this chapter by considering the influential work of Hartry Field. Although there are other nominalist philosophies of mathematics addressing the indispensability argument, ${ }^{1}$ I shall restrict my attention to Field's distinctive fictionalism. There are many reasons for this, not least of which is the considerable influence Field's work has had on the literature in the 17 years since the publication of Science Without Numbers [Fie 80]. This influence is no accident; it's a tribute to the plausibility of the account of mathematics offered by Field, and his unwillingness to dodge the issues associated with the applications of mathematics. Furthermore, unlike other nominalist philosophies of mathematics, ${ }^{2}$ Field's nominalism is not revisionist:

I do not propose to reinterpret any part of classical mathematics; instead, I propose to show that the mathematics needed for application to the physical world does not include anything which even prima facie contains references

\footnotetext{
${ }^{1}$ See [Bur 97] for detailed discussion of the various nominalist programs.

${ }^{2}$ For example, Chihara [Chi 73], where mathematical discourse is reinterpreted so as to be about linguistic entities rather than mathematical entities.
} 
to (or quantifications over) abstract entities like numbers, functions, or sets. Towards that part of mathematics which does contain references to (or quantification over) abstract entities-and this includes virtually all of conventional mathematics-I adopt a fictional attitude: that is, I see no reason to regard this part of mathematics as true. [Fie 80,1-2]

He accepts the Quinean backdrop discussed in chapter 2 and agrees that if mathematics were indispensable to our best scientific theories we would have good cause to grant mathematical entities real status, but he denies that mathematics is indispensable to science. In effect he accepts the burden of proof in this debate. That is, he accepts that he must show (i) how it is that mathematical discourse may be used in its various applications in physical science and (ii) that it is possible to do science without reference to mathematical entities. This is indeed an ambitious project and certainly one deserving careful attention, for if he succeeds indispensability theory is no longer a way of motivating mathematical realism.

Given that I am committed to a defence of the indispensability argument, it may come as some surprise to find that my disagreements with Field are rather slight. We both accept the Quinean backdrop of holism and naturalism; we both agree that the indispensability argument is a good argument - indeed we both agree that it is the only good argument for Platonism; we both agree that if mathematics can be shown to be dispensable to science (and its utility to science adequately explained) then we would have no reason to believe in the existence of mathematical entities. What we disagree on is the question of whether mathematics is or is not dispensable to science. We both believe that this last issue is still open, but whereas Field thinks enough of the nominalisation of science has been carried out to warrant the tentative conclusion that mathematics is dispensable, I'm inclined to think a lot more work needs to be done to warrant such a conclusion (even tentatively). In this chapter I hope to make clear what some of this extra work is.

In the next section I will briefly outline Field's program. In the following two sections I will re-examine the indispensability argument, with particular emphasis on the notion of 'indispensability' itself. I will argue that (i) dispensability cannot be "cashed out" purely in terms of eliminability, as is often assumed, and (ii) if an entity is dispensable to a theory, all talk of that entity must be eliminable from the theory and the resulting theory be preferable to the original. ${ }^{3}$ In particular,

\footnotetext{
${ }^{3}$ John Burgess in [Bur 83] argues for a similar conclusion in his discussion of revolutionary nominalism. The difference though, is that Burgess sees the question of theory choice, between Platonistic and nominalistic scientific theories, as a separate issue from that of indispensability,
} 
I will emphasize the important role non-empirical criteria of confirmation theory play in deciding whether a given entity is indispensable or not. In section 4.4 I'll look at some case studies in the history of science where it seems implausible that mathematics could turn out to be dispensable in the sense just outlined. In the final section I will assess the importance of these considerations for Field's program, concluding that while they certainly do not undermine his program in any significant way, they do make it clear that there is more to be done than at least some of his supporters appreciate.

\subsection{The Science Without Numbers Project}

Before discussing the details of Field's project it is important to understand something of its motivation. Field is driven by two things. Firstly, there are well known prima facie difficulties with Platonism - namely the two Benacerraf problems mentioned in the first chapter of this thesis (and which will be discussed in more detail in the final chapter) - which nominalism avoids [Fie 89, 6]. ${ }^{4}$ Secondly, he is motivated by certain rather attractive principles in the philosophy of science: (i) that we ought to seek intrinsic explanations whenever this is possible and (ii) that we ought to eliminate arbitrariness from theories [Fie 80, ix]. In relation to (i), Field says "one wants to be able to explain the behaviour of the physical system in terms of the intrinsic features of that system, without invoking extrinsic entities (whether nonmathematical or mathematical) whose properties are irrelevant to the behaviour of the system being explained" [Fie 84, 193]. He also points out that this concern is orthogonal to nominalism [Fie 80,44]. As for (ii), this too is independent of nominalism. Co-ordinate independent (tensor) methods used in most field theories are considered more attractive by Platonists and nominalists alike. The importance of these motivations will arise in the course of this chapter. At this stage it is sufficient to note them and, in particular, to realise that his project is driven by more than just nominalist concerns. ${ }^{5}$

Now to the details of Field's project. There are two parts to the project. The first is to justify the use of mathematics in its various applications in empirical science. If one is to present a believable, fictional account of mathematics, one must

whereas I see it as part of what we mean by 'indispensability'.

${ }^{4} \mathrm{Or}$, rather, nominalism trades these problems for a different set of problems-most notably, to disarm the indispensability argument.

${ }^{5}$ Indeed, in private conversation Field suggested that his continued interest in this project stems more from (i) and (ii) than the nominalist motivation. 
present some account of how mathematics may be used with such effectiveness in its various applications in physical theories. To do this Field argues that mathematical theories don't have to be true to be useful in applications, they merely need to be conservative. This is, roughly, that if a mathematical theory is added to a nominalist scientific theory, no nominalist consequences follow that wouldn't follow from the scientific theory alone. I'll have more to say about this shortly. The second part of his program is to demonstrate that our best scientific theories can be suitably nominalised. To do this he is content to nominalise a large fragment of Newtonian gravitational theory. Although this is a far cry from showing that all our current best scientific theories can be nominalised, it is certainly not trivial. The hope is that once one sees how the elimination of reference to mathematical entities can be achieved for a typical physical theory, it will seem plausible that the project could be completed for the rest of science.

One further point which is important to bear in mind is that Field is interested in undermining what he takes to be the only good argument for Platonism. He is thus justified in using Platonistic methods. His strategy is to show Platonistically that abstract entities are not needed in order to do empirical science. If his program is successful, "platonism is left in an unstable position: it entails its own unjustifiability" [Fie 80,6]. I'll now discuss the first part of his program, as I've outlined it.

\subsubsection{Conservativeness}

Field's account of how it is that mathematical theories might be used in scientific theories, even when the mathematical theory in question is false, is crucial to his fictional account of mathematics. Field, of course, does provide such an account, the key to which is the concept of conservativeness, which may be defined (roughly) as follows: ${ }^{6}$

Definition 1 (Conservativeness) A mathematical theory $M$ is said to be conservative if, for any body of nominalistic assertions $S$ and any particular nominalistic assertion $C$, then $C$ is not a consequence of $M+S$ unless it is a consequence of $S$.

\footnotetext{
${ }^{6}$ I say 'roughly' because the following definition needs refinement in order to avoid certain technical difficulties. For example, we need to exclude the possibility of the nominalistic theory containing the assertion that there are no abstract entities. Such a situation would render $M+S$ inconsistent. There are natural ways of performing the refinements required, but the details aren't important here. See [Fie 80, 11-12] for details.
} 
A couple of comments are warranted here in relation to definition 1. Firstly, nominalistic assertion is taken to mean an assertion in which all the variables are explicitly restricted to non-mathematical entities (for reasons I suggested in footnote 6). Secondly, Field is at times a little unclear about whether he is speaking of semantic entailment or syntactic entailment (e.g. [Fie 80, 16-19]); in other places (e.g. [Fie 80, 40, footnote 30] and [Fie 85]) he is explicit that it is semantic entailment he is concerned with. Thirdly, the key concept of conservativeness is closely related to (semantic) consistency. ${ }^{7}$ Field, however, cannot (and does not) cash out consistency in model-theoretic terms (as is usually the case), for obviously such a construal depends on models, and these are not available to a nominalist. Instead, Field appeals to a primitive sense of possibility. ${ }^{8}$

Now if it could be proved that all of mathematics were conservative, then its truth or falsity would be irrelevant to its use in empirical science. More specifically, if some mathematical theory were false but conservative, it would not lead to false nominalistic assertions when conjoined with some nominalist, empirical theory, unless such false assertions were consequences of the empirical theory alone. As Field puts it "mathematics does not need to be true to be good" [Fie 85, 125]. Put figuratively, conservativeness ensures that the alleged falsity of the mathematical theory does not "infect" the theory as a whole.

Field provides a number of reasons for thinking that mathematical theories are conservative. These reasons include several formal proofs of the conservativeness of set theory. ${ }^{9}$ Here I just wish to demonstrate the plausibility of the conservativeness claim by showing how closely related conservativeness is to consistency. Firstly, for pure set theory (i.e. set theory without urelements ${ }^{10}$ ) conservativeness follows from consistency alone [Fie 80, 13]. In the case of impure set theory the conservativeness claim is a little stronger than consistency. An impure set theory could be consistent but fail to be conservative because it implied conclusions about concrete entities that were not logically true. Field sums up the situation:

[S] tandard mathematics might turn out not to be conservative [...], for it might conceivably turn out to be inconsistent, and if it is inconsistent it certainly isn't conservative. We would however regard a proof that standard mathematics was

\footnotetext{
${ }^{7}$ Conservativeness entails consistency and, in fact, conservativeness can be defined in terms of consistency.

${ }^{8}$ This last point will be important for the discussion of section 6.4

${ }^{9}$ See [Fie 80, 16-19] and [Fie 92] for details.

${ }^{10} \mathrm{~A}$ urelement is an element of a set that is not itself a set.
} 
inconsistent as extremely surprising, and as showing that standard mathematics needed revision. Equally, it would be extremely surprising if it were to be discovered that standard mathematics implied that there are at least $10^{6}$ nonmathematical objects in the universe, or that the Paris Commune was defeated; and were such a discovery to be made, all but the most unregenerate rationalists would take this as showing that standard mathematics needed revision. Good mathematics is conservative; a discovery that accepted mathematics isn't conservative would be a discovery that it isn't good. [Fie 80, 13]

It is also worth noting that Field claims that there is a disanalogy here between mathematical theories and theories about unobservable physical entities. The latter he suggests do facilitate new conclusions about observables and hence are not conservative [Fie 80,10]. This disanalogy is due to the fact that conservativeness is also closely related to necessary truth. In fact, conservativeness follows from necessary truth. Field's remark that "[c]onservativeness might loosely be thought of as "necessary truth without the truth'" [Fie 88, 241] is very helpful. As is the following diagram (which I also borrow from Field [Fie 88, 241]), illustrating the relevant relationships:

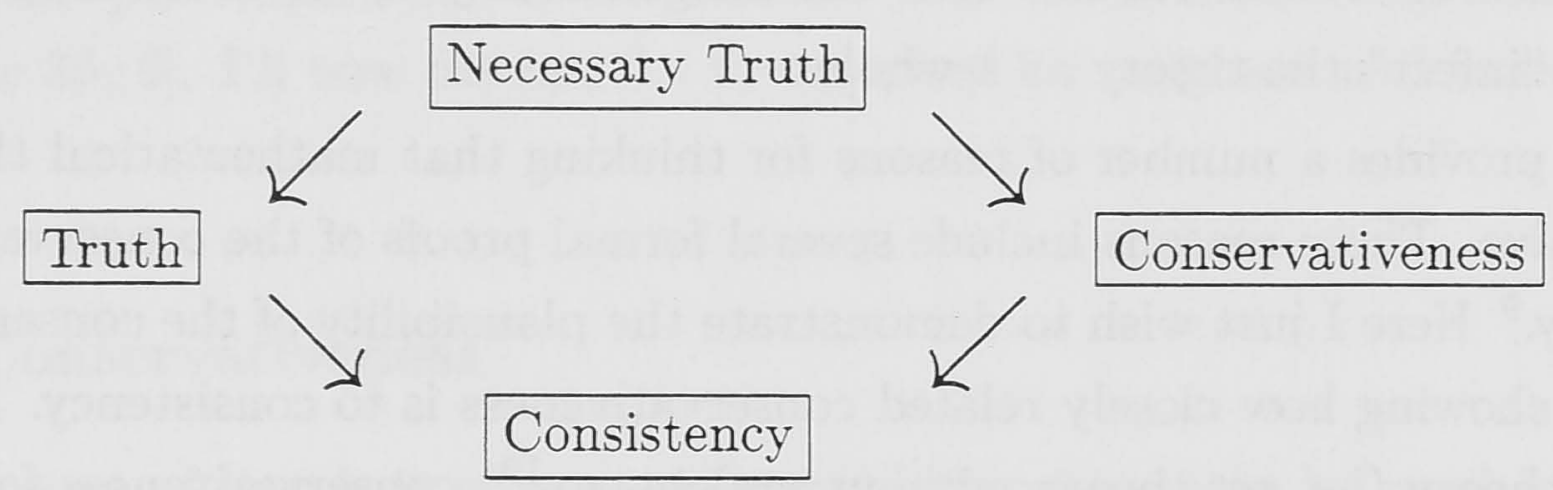

With conservativeness established then, it is permissible for a fictionalist about mathematics to use mathematics in a nominalistic theory, despite the falsity of the former. It remains to show that our best current scientific theories can be purged of their references to abstract objects. I'll discuss Field's treatment of this in the next section.

\subsubsection{Nominalisation}

Field's strategy for eliminating all references to mathematical objects from empirical science is to appeal to the representation theorems of measurement theory. Although the details of this are highly technical, no account of Field's program is complete without at least an indication of how this is done. It is also of considerable interest in its own right. Furthermore, as Michael Resnik points out, this part of his program 
provides a very nice account of applied mathematics which should be of interest to all philosophers of mathematics, realists and anti-realists alike [Res 83, 515]. In light of all this it would be remiss of me not to at least outline this part of Field's program, despite its limited significance to the main body of this thesis.

Perhaps the most accessible example of a representation theorem is seen in Hilbert's axiomatisation of Euclidean geometry [Hil 99], on which Field's program is modelled. The central idea is to replace all talk of distance and location, which require quantification over real numbers, with the comparative predicates 'between' and 'congruent', which require only quantification over space time points. It will be instructive to present this case in a little more detail. My treatment here follows Field [Fie 80, 24-29].

For the present purposes, the important feature of Hilbert's theory is that it contains the following relations:

1. The three-place between relation (where ' $y$ ' is between ' $x$ ' and ' $z$ ' is written ' $y$ Bet $x z^{\prime}$ ) which is intuitively understood to mean that $x$ is a point on the line segment with endpoints $y$ and $z$.

2. The four-place segment-congruence relation (where ' $x$ and $y$ are congruent to $z$ and $w$ ' is written ' $x y$ Cong $z w$ ') which is intuitively understood to mean that the distance from point $x$ to point $y$ is the same as the distance from point $z$ to point $w$.

The notion of (Euclidean) distance appealed to in the segment-congruence relation is not part of Hilbert's theory; in fact it cannot even be defined in the theory. But this does not mean that Hilbert's theory is deficient in any sense, for he proved in a broader mathematical theory the following representation theorem:

Theorem 1 (Hilbert's Representation Theorem) For any model of Hilbert's axiom system for space $S$, there exists a function $\mathrm{d}: S \times S \rightarrow \mathbb{R}^{+} \cup\{0\}$ which satisfies the following two homomorphism conditions:

(a) For any four points $x, y, z$ and $w, x y$ Cong $z w$ iff $\mathrm{d}(x y)=\mathrm{d}(z w)$;

(b) For any three points $x, y$ and $z$, $y$ Bet $x z$ iff $\mathrm{d}(x y)+\mathrm{d}(y z)=\mathrm{d}(x z)$.

From this it is easy to show that any Euclidean theorem about length would be true if restated as a theorem about any function d satisfying the conditions of theorem 1 . In this way we can replace quantification over numbers with quantification over points. As Field puts it: 
So in the geometry itself we can't talk about numbers, and hence we can't talk about distances [...]; but we have a metatheoretic proof which associates claims about distances [...] with what we can say in the theory. Numerical claims then, are abstract counterparts of purely geometric claims, and the equivalence of the abstract counterpart with what it is an abstract counterpart of is established in the broader mathematical theory. [Fie 80, 27]

Hilbert also proved a uniqueness theorem corresponding to theorem 1 . This theorem states that if there are two functions $\mathrm{d}_{1}$ and $\mathrm{d}_{2}$ satisfying the conditions of theorem 1 , then $\mathrm{d}_{1}=k \mathrm{~d}_{2}$ where $k$ is some arbitrary positive constant. This, claims Field, provides a satisfying explanation of why geometric laws formulated in terms of distance are invariant under multiplication by a positive constant (and that this is the only transformation under which they are invariant). Field claims that this is one of the advantages of this approach, in that this fact is given an intrinsic explanation in terms of the intrinsic facts about space [Fie 80, 27]. I'll have more to say about this later in the chapter.

With the example of Hilbert's axiomatisation of Euclidean space in hand, Field then does for Newtonian space-time what Hilbert did for $\mathbb{R}^{2}$. This in itself is non-trivial, but Field is required to do much more, since he must dispense with all mention of physical quantities. He does this by appeal to relational properties which compare space-time points with respect to the quantity in question. For example, rather than saying that some space-time point has a certain gravitational potential, Field compares space-time points with respect to the 'greater gravitational potential' relation. ${ }^{11}$ The details of this and the more technical task of how to formulate differential equations involving scalar quantities (such as gravitational potential) in terms of the spatial and scalar relational primitives need not concern us here. (The details can be found in [Fie 80,55-91].) The important point is that Field is able to derive an extended representation theorem which, for the sake of completeness, I state here without further comment: ${ }^{12}$

Theorem 2 (Field's Extended Representation Theorem) For any model of a theory $N$ with space-time $S$ that uses comparative predicates but not numerical functors there are:

\footnotetext{
${ }^{11}$ Of course there is the task of getting the axiomatisation of the gravitational potential relation such that the desired representation and uniqueness theorems are forthcoming. But much of Field's work has, in effect, been done for him by workers in measurement theory [Fie 80, 57-58].

${ }^{12}$ The statement of the theorem here is derived from [Fie 85, 130-131].
} 
(a) a 1-1 spatio-temporal co-ordinate function $\Phi: S \rightarrow \mathbb{R}^{4}$, which is unique up to generalised Galilean transformation,

(b) a mass density function $\rho: S \rightarrow \mathbb{R}^{+} \cup\{0\}$, which is unique up to a positive multiplicative transformation, and

(c) a gravitation potential function $\Psi: S \rightarrow \mathbb{R}$, which is unique up to positive linear transformation,

all of which are structure preserving (in the sense that the comparative relations defined in terms of these functions coincide with the comparative relations used in $N)$; moreover, the laws of Newtonian gravitational theory in their functorial form hold if $\Phi, \rho$ and $\Psi$ are taken as denotations of the relevant functors.

We have now seen enough to get the flavour of Field's program, so I'll move on to discuss some related philosophical issues.

There are many complaints against Field's program, ranging from the complaint that it is not genuinely nominalist [Res $85 \mathrm{a}$, Res $85 \mathrm{~b}$ ] since it makes use of spacetime points, to technical difficulties such as the complaint that it is hard to see how Field's program can be made to work for theories where the represented objects are not space-time points, but mathematical objects themselves [Mal 82]. ${ }^{13}$ While such debates are not without interest, I will not discuss them in any detail here. Instead, I wish to clear up the issue of exactly what is required of Field's program. This issue is occasionally confused in the literature, where it is sometimes claimed that Field's program is simply that of eliminating quantification over mathematical entities. ${ }^{14}$ Although Field does appreciate that the goal of the program is more than that of elimination, the point is important and worth stressing for the benefit of some of his supporters.

The problem is that it is not clear how the word 'indispensable' is to be understood in this debate. In order to understand just what is required of a program like Field's then, or indeed to understand the full force of the indispensability argument, we must first clear up what we mean by 'indispensable'. I shall discuss this in some detail in the following section.

\footnotetext{
${ }^{13}$ For example, in classical Hamiltonian mechanics the represented objects are possible dynamical states. Similar problems, it seems, will arise in any "phase-space" theory, and the prospects look even dimmer for quantum mechanics [Mal 82, 533-534].

${ }^{14}$ For instance, David Papineau seems to endorse this view when he suggests that the nominalisation part of Field's program requires describing the natural world without mentioning abstract objects [Pap 93, 193].
} 


\subsection{What is it to be Indispensable?}

Recall that the indispensability argument may be stated as follows: We have good reason to believe our best scientific theories and there are no grounds on which to differentiate scientific entities from mathematical entities, so we have good reason to believe in mathematical entities, since they, like the relevant scientific entities, are indispensable to the theories in which they occur. Furthermore, it is exactly the same evidence that confirms the scientific theory as a whole, that confirms the mathematical portion of the theory and hence the mathematical entities contained therein. We have just seen that part of Field's program is to show that mathematics is in fact dispensable to science. He begins this task by giving a nominalistic treatment of Newtonian gravitational theory, thus allegedly showing that mathematical entities are not indispensable to this theory. This is the point that motivates the present section. He does not give a clear account of what he takes 'dispensable' to mean in this context. He clearly does not take it to mean simply eliminable ${ }^{15}$ and yet most of his work is devoted to showing that mathematical entities are eliminable from physical theories.

This failure to explicate what is meant by 'indispensable' in Quine's argument allows programs such as Field's to look more attractive than they perhaps ought. ${ }^{16}$ In order to come to a clear understanding of how 'indispensability' is to be understood, I will consider a case where there should be no disagreement about the dispensability of the entity in question. I shall then analyse this case to see what leads us to conclude that the entity in question is dispensable.

Consider an empirically adequate and consistent theory $\Gamma$ and let ' $\xi$ ' be the name of some entity neither mentioned, predicted, nor ruled out by $\Gamma$. Clearly we can construct a new theory $\Gamma^{+}$from $\Gamma$ by simply adding the sentence ' $\xi$ exists' to $\Gamma$. It is reasonable to suppose that $\xi$ plays no role in the theory $\Gamma^{+},{ }^{17}$ it is merely predicted by it. I propose that there should be no disagreement here when I say that $\xi$ is dispensable to $\Gamma^{+}$, but let us investigate why this is so.

\footnotetext{
${ }^{15}$ For example, in Science Without Numbers Field suggest that mathematical entities are not theoretically indispensable since "we can give attractive reformulations of the theories of modern physics] in which mathematical entities play no role" (my emphasis) [Fie 80, 8].

${ }^{16}$ Quine actually speaks of entities existentially quantified over in the canonical form of our best theories, rather than indispensability. (See [Qui 48] for details.) Still, the debate continues in terms of indispensability, so we would be well served to clarify this latter term.

${ }^{17}$ The reason I hedge a bit here is that if $\Gamma$ asserts that all entities have positive mass, for instance, then the existence of $\xi$ automatically helps account for some of the "missing mass" of the universe Thus $\xi$ does play a role in $\Gamma^{+}$. I know of no way of ruling out such cases; hence the hedge.
} 
On one interpretation of 'dispensable' we could argue that $\xi$ is not dispensable since its removal from $\Gamma^{+}$results in a different theory, namely $\Gamma$. This is not a very helpful interpretation though, since all entities are indispensable to the theories in which they occur under this reading. Another interpretation of 'dispensable' might be that $\xi$ is dispensable to $\Gamma^{+}$since there exists another theory, $\Gamma$, with the same empirical adequacy as $\Gamma^{+18}$ in which $\xi$ does not occur. This interpretation can also be seen to be inadequate since it may turn out that no theoretical entities are indispensable under this reading. This result follows from Craig's theorem. ${ }^{19}$ If the vocabulary of the theory can be partitioned in the way that Craig's theorem requires (cf. footnote 19), then the theory can be reaxiomatised so that any given theoretical entity is eliminated. ${ }^{20} \mathrm{I}$ claim, therefore, that this interpretation of 'dispensable' is unacceptable since it fails to account for why $\xi$ in particular is dispensable.

This leads me to the following explication of 'dispensable':

Definition 2 (Dispensable) An entity is dispensable to a theory iff the following two conditions hold:

(i) There exists a modification of the theory in question resulting in a second theory with exactly the same observational consequences as the first, in which the entity in question is neither mentioned nor predicted.

(ii) The second theory must be preferable to the first.

In the preceding example, then, $\xi$ is dispensable since $\Gamma$ makes no mention of $\xi$ and $\Gamma$ is preferable to $\Gamma^{+}$in that the former has less ontological commitment than the latter, all other things being equal. (Assuming, of course, that less ontological commitment is better. ${ }^{21}$ )

\footnotetext{
${ }^{18}$ Modulo my concerns in footnote 17.

${ }^{19}$ This theorem states that relative to a partition of the vocabulary of an axiomatisable theory $T$ into two classes, $\tau$ and $\omega$ (theoretical and observational say) there exists an axiomatisable theory $T^{\prime}$ in the language whose only non-logical vocabulary is $\omega$, of all and only the consequences of $T$ that are expressible in $\omega$ alone.

${ }^{20}$ Naturally the question of whether such partitioning is possible is important and somewhat controversial. Quine (arguably) would deny that such a partition is possible. If he is right about this, it will be considerably more difficult to eliminate theoretical entities from scientific theories. I'm willing to grant for the sake of argument, at least, that such a partitioning is possible.

${ }^{21}$ One way in which you might think that less ontological commitment is not better, is if $\xi$ actually exists. In this case it seems that $\Gamma^{+}$is the better theory since it best describes reality. This, however, is to gloss over the important question of how we come to know that $\xi$ exists. If there is some evidence of $\xi$ 's existence then $\Gamma^{+}$will indeed be the better theory, since it will be empirically superior. If there is no such evidence for the existence of $\xi$ then it seems entirely reasonable to prefer $\Gamma$ over $\Gamma^{+}$as I suggest. It is the latter I had in mind when I set up this case.
} 
Now it might be argued that on this reading once again every theoretical entity is dispensable, since by Craig's theorem we can eliminate any reference to any entity and the resulting theory will be better, since it doesn't have ontological commitment to the entity in question. This argument is flawed though, since the reason for preferring one theory over another is a complicated question-it is not simply a matter of empirical adequacy combined with a principle of ontological parsimony. In the next section I will discuss some aspects of confirmation theory and what role it plays in indispensability decisions.

\subsection{The Role of Confirmation Theory}

Confirmation theory is the study of those principles which guide scientific reasoning other than reasoning of the deductive kind. In particular it will help us in deciding whether one theory is better than another by giving us some desiderata for "good theories".

Firstly and foremost, a "good theory" must be empirically adequate, that is, it must agree with all (or at least most) observation. Secondly, it must be consistent, both internally and with other major theories. This is not the whole story though. As we have already seen, $\Gamma$ and $\Gamma^{+}$have the same degree of empirical adequacy and consistency (by construction) and yet we are inclined to prefer the former over the latter. I am in agreement with many authors here ${ }^{22}$ that amongst the additional features we require are the following:

(i) Simplicity/Parsimony: Given two theories with the same empirical adequacy, we generally prefer that theory which is simpler both in its statement and in its ontological commitments. For example, Einstein, in his formulation of the special theory of relativity, refused to admit an undetectable luminiferous ether, as some rival theories did, to jointly explain the propagation of electromagnetic radiation through apparently empty space and the failure of the Michelson-Morley experiment to detect such an ether [Ein 05, 38].

(ii) Unificatory/Explanatory Power: Philip Kitcher argues rather convincingly in [Kit 81] for scientific explanation being unification; that is, accounting

Indeed the former case is ruled out by construction. I am not concerned with whether $\xi$ actually exist or not-just that there be no evidence for it.

${ }^{22}$ See, for instance, Hempel [Hem 65, 203-206], Horwich [Hor 82, 1-15], Weinberg [Wei 93, 105131], Glymour [Gly 80, 152-155], and Quine in [Qui 60a]. While all these authors wouldn't agree with my characterisation of the additional features entirely, I think I have captured what are the most common elements in all their accounts. 
for a maximum of observed phenomena with a minimum of theoretical devices. Whether or not you accept Kitcher's account, we still require that a theory not simply predict certain phenomena, but explain why such predictions are expected. Furthermore, the best theories do so with a minimum of theoretical devices. For example, the success of Newtonian gravitational theory was in no small way due to its ability to explain such diverse phenomena as tides, planetary orbits and projectile motion (among other things) from a small stock of theoretical "machinery".

(iii) Boldness/Fruitfulness: We expect our best theories not to simply predict everyday phenomena, but to make bold predictions of novel entities and phenomena which lead to fruitful future research. The prediction of gravitational waves by general relativity is an example of a bold prediction that is still being actively investigated.

(iv) Formal Elegance: This is perhaps the hardest feature to characterise (and no doubt the most contentious). However, there is at least some sense in which our best theories have aesthetic appeal. For instance, it may well be on the grounds of formal elegance that we rule out ad hoc modifications to a failing theory.

I will not argue in detail for each of these, except to say that despite the notorious difficulties involved in explicating what we mean by such terms as 'simplicity' and 'elegance', we do look for such virtues in our best theories. Otherwise we could never choose between two theories such as $\Gamma$ and $\Gamma^{+}$. I do not claim that this list is comprehensive nor do I claim that it is minimal; ${ }^{23}$ I merely claim that these sorts of criteria are typically appealed to in the literature to distinguish "good theories" and I have no objection to such appeals.

In the light of the preceding discussion then, we see that to claim that an entity is dispensable is to claim that a modification of the theory in which it is posited can be made in such a way as to eliminate the entity in question and result in a theory which is better overall (or at least not worse) in terms of simplicity, elegance and so on. Thus we see that the argument I presented at the end of the previous section that any theoretical entity is dispensable does indeed fail, as I claimed. This is because in most cases the benefit of ontological simplicity obtained by the elimination of the entity in question will be more than offset by losses in other areas.

\footnotetext{
${ }^{23}$ For instance it may be possible to explain formal elegance in terms of simplicity and unificatory power.
} 
While it seems reasonable to suppose that the elimination from the body of scientific theory of physical entities such as electrons would cause an overall reduction in the previously described virtues of that theory, it is not so clear that the elimination of mathematical entities would have the same impact. Someone might argue that mathematics is certainly a very effective language for the expression of scientific ideas, in that it simplifies the calculations and statement of much of science, but to do so at the expense of introducing into one's ontology the whole gamut of mathematical entities is just not a good deal.

One response to this is to simply deny that it is a high price at all. After all, a powerful and efficient language is the cornerstone of any good theory. If you have to introduce a few more entities into the theory to get it, then so be it. Although, admittedly, I have considerable sympathy with this response, in this thesis I wish to pursue a different, and I think, a more convincing line. I will argue that mathematics plays an active role in many of the theories which make use of it. That is, it is not just a tool which makes calculations easier or simplifies the statement of the theory; it makes important contributions to all of the desiderata of good theories I mentioned earlier. $^{24}$

My strategy from here is to show that there is good reason to believe that the mathematised version of a theory is more "virtuous" than the original theory, and so there is good reason to believe mathematics is indispensable, in the sense I have previously outlined, to our best physical theories. I shall demonstrate this in the next section by appealing to a number of examples of physical theories and showing how the mathematised theory is capable of more than what one would expect from a nominalised version. Notice that it is not necessary for me to show that this is the case for all our best physical theories, as the indispensability argument goes through in case mathematics is indispensable to some non-empty subset of our best physical theories.

I also note here that I am not proposing to prove the indispensability of mathematics in the cases I consider; merely to suggest that if these theories were stripped of their mathematical content it seems that they would lose much of their appeal. If I succeed, then the burden of proof lies with anyone who claims that mathematics is dispensable, for they must show, firstly, how it is possible to remove all commitment to mathematical entities from all physical theories, and secondly, how this

\footnotetext{
${ }^{24}$ The mathematical physicist Freeman Dyson makes a similar point when he says "...mathematics is not just a tool by means of which phenomena can be calculated; it is the main source of concepts and principles by means of which new theories can be created" [Dys 64, 129].
} 
removal does not result in a reduction of virtue of these theories. This problem will be particularly evident in the cases I consider in the next section.

\subsection{The Role of Mathematics in Physical Theories}

David Papineau claims that "the incorporation of pure mathematics into scientific theories [...] might make it easier to do calculations, but [...] receives no backing from principles of scientific theory choice" [Pap 93, 196]. In this section I will show that this view is, at the very least, extremely controversial. I will demonstrate this by appealing to a number of examples in which mathematics contributes to the unification and boldness of the physical theory in question, and therefore is supported by well recognised principles of scientific theory choice.

\subsubsection{The Complex Numbers}

The first example is the complex numbers. I will show how the introduction of these was responsible for a great deal of unification within both pure mathematics and in more practical areas such as differential equations in physics.

Perhaps the most profound use of complex numbers is in the theory of integration of complex valued functions of a complex variable; in particular, in providing a method for the exact evaluation of many real integrals which are unobtainable by other methods. ${ }^{25}$ This, however, would take us too far into the theory of complex analysis for our present purposes. Instead, I wish to look at a simpler application of complex numbers: the unification of the exponential and trigonometric functions. This has direct applications in the study of second-order ordinary differential equations which arise in almost all branches of science, including fluid mechanics, heat conduction and population dynamics.

We begin by introducing the number $i=\sqrt{-1}$ and defining a complex variable $z=x+y i$ where $x$ and $y$ are real. Once we have extended the operations ' + ' and '.' and the relation '=' from the reals to the complex in the natural way, we can introduce complex exponentiation via the following equation, known as Euler's formula:

$$
e^{i \theta}=\cos \theta+i \sin \theta \quad \theta \in \mathbb{R} .
$$

\footnotetext{
${ }^{25}$ I am referring here to the remarkable Cauchy integral formula and the like. See Ahlfors' classic treatment of this subject in [Ahl 66] for further details.
} 
From this we can define the trigonometric functions for a complex variable $z$ as

$$
\sin z=\frac{e^{i z}-e^{-i z}}{2 i} \text { and } \cos z=\frac{e^{i z}+e^{-i z}}{2}
$$

The usual real-valued sine and cosine functions are then seen to be special cases. Thus the complex numbers are instrumental in the unification of the trigonometric and the exponential functions. This unification, being within mathematics itself, may seem somewhat artificial, so I shall demonstrate how this unification "flows through" to physics.

Consider the second-order linear homogeneous ordinary differential equation with constant coefficients:

$$
y^{\prime \prime}+y^{\prime}+y=0
$$

where $y$ is a real-valued function of the single real variable $x$. Equations such as these are solved by considering their characteristic equations, which are quadratics and so, by the fundamental theorem of algebra, always have two (complex) roots (counting multiplicity). In this case the characteristic equation is

$$
r^{2}+r+1=0
$$

which has (complex) roots $r=-\frac{1}{2} \pm i \frac{\sqrt{3}}{2}$. Now the general solution to an equation with unequal roots to its characteristic equation is:

$$
y=c_{1} e^{r_{1} x}+c_{2} e^{r_{2} x}
$$

where $c_{1}$ and $c_{2}$ are arbitrary real constants and $r_{1}$ and $r_{2}$ are the two distinct roots of the characteristic equation. ${ }^{26}$ Note that (4.2) is indifferent as to whether $r_{1}$ and $r_{2}$ are real or complex. Thus the solution to (4.1) is given by

$$
y=c_{1} e^{\left(-\frac{1}{2}+\frac{\sqrt{3}}{2} i\right) x}+c_{2} e^{\left(-\frac{1}{2}-\frac{\sqrt{3}}{2} i\right) x}
$$

which reduces to the real solution

$$
y=e^{\frac{-x}{2}}\left(c_{1} \cos \left(\frac{\sqrt{3}}{2} x\right)+c_{2} \sin \left(\frac{\sqrt{3}}{2} x\right)\right)
$$

due to Euler's formula given previously.

Thus we see that without the use of complex numbers we would have to treat the equations $y^{\prime \prime}-y=0$, which has real roots to its characteristic equation, quite

\footnotetext{
${ }^{26}$ See [Boy 86] for further details.
} 
differently from $y^{\prime \prime}+y=0$, which has complex roots to its characteristic equation. Furthermore, the connection between the exponential function, which is a solution to the first, and the cosine and sine functions, which are solutions to the second, is spelled out via the definitions of trigonometric functions of a complex variable given earlier. This I see as a fine example of the unity a mathematical theory may bring, not only to other mathematical theories, but also to scientific theory as a whole.

What is more, I take this unity to be not simply an algorithmic unity; that is, a single method for finding solutions to these equations. Rather, I take it that the algorithmic unity arises out of deep structural similarities between the systems portrayed by these equations. For example, if two different physical systems are governed by the same differential equation, it's clear that there is some similarity between these systems, no matter how disparate the systems may seem (just as a red planet and a red dog have something in common). It seems plausible, at least, that this similarity is structural and is captured by the relevant differential equation. Even when the systems are governed by different differential equations, structural similarities may still be revealed in the mathematics. In the case of the two equations in the previous paragraph, the structural similarity of any two systems governed by these equations is revealed by the connection between the equation's respective solutions. Mathematics, because of its abstract nature, is extremely well suited to providing unification in this very important sense.

\subsubsection{Maxwell's Equations}

In this and the next example I will show that mathematics may contribute to the boldness of theories by bearing the prime responsibility in the prediction of novel phenomena. In this example we will see how Maxwell's equations predicted electromagnetic radiation.

Maxwell found that the accepted laws for electromagnetic phenomena prior to about 1864, namely Gauss's law for electricity, Gauss's law for magnetism, Faraday's law and Ampère's law, jointly contravened the conservation of electric charge. He thus modified Ampère's law to include a "displacement current", which was not an electric current in the usual sense (a so called "conduction" current), but a rate of change (with respect to time) of an electric field. This modification was made on the basis of formal mathematical analogy, not on the basis of empirical evidence. The analogy is of course with (Newtonian) gravitational theory's conservation of mass principle, which is best understood mathematically as a symmetry requirement. The 
modified Ampère's law states that the "curl" of a magnetic field is proportional to the sum of the "conduction" current and the "displacement current". ${ }^{27}$ When this law (known as the Maxwell-Ampère law) replaces the original Ampère's law in the above set of equations, they are known as Maxwell's equations and they provide a wonderful unity to the subject of electromagnetism.

The interesting part of this story, for the purposes of the present discussion though, is that Maxwell's equations were formulated based on the assumption of stationary charges and charges moving with a constant velocity, and yet such was Maxwell's faith in the equations, he claimed that they would hold for any arbitrary system of electric fields, currents, and magnetic fields. In particular they would hold for charges with accelerated motion and for systems with zero "conduction" current. Thus he predicted the phenomenon of electromagnetic radiation, which was a wave capable of propagating in a vacuum. Furthermore, it is a consequence of these equations that the speed of propagation of this radiation is the speed of light. This was the first evidence that light was an electromagnetic phenomenon.

I claim that these predictions, which, incidentally, were confirmed experimentally by Heinrich Hertz in 1888 (nine years after Maxwell's death), can be largely attributed to the mathematics, since the predictions were being made for circumstances beyond the basal assumptions of the equations' formulation. Furthermore, the formulation of the crucial equation (the Maxwell-Ampère law) for these predictions was based on formal mathematical analogy. ${ }^{28}$

\subsubsection{The Dirac Equation}

The next example is another case where the mathematical component of the theory played an active part in the prediction of novel phenomena-in this case the discovery of antimatter. Here, however, there was reason to doubt the predictions, not because the theory was being used beyond the scope of its base assumptions, but because the solutions to the equations involved didn't seem to have any physical representation.

In classical physics one occasionally comes across solutions to equations which are discarded because they are taken to be "non-physical". Examples include negative

\footnotetext{
${ }^{27}$ More precisely, $\nabla \times \mathbf{B}=\frac{4 \pi}{c} \mathbf{J}+\frac{1}{c} \frac{\partial}{\partial t} \mathbf{E}$ where $\mathbf{E}$ and $\mathbf{B}$ are the electric and magnetic field vectors respectively, $\mathbf{J}$ is the current density, and $c$ is a constant (the speed of light in a vacuum).

${ }^{28} \mathrm{I}$ am reminded of a quotation from Richard Feynman here: "When you get it right, it is obviously right... because usually what happens is that more comes out than goes in" [Fey 65, 171] The "something more" here is electromagnetic radiation.
} 
energy solutions to dynamical systems. This situation arose for Paul Dirac in 1928 when he was studying the solutions of the equation of relativistic quantum mechanics which now bears his name. This equation describes the behaviour of electrons and hydrogen atoms, but was found to also describe particles with negative energies. It must have been tempting for Dirac to simply dismiss such solutions as "nonphysical"; however, strange things are known to occur in quantum mechanics, and intuitions about what is "non-physical" are not so clear. So Dirac investigated the possibility of negative energy solutions and, in particular, to give an account of why a particle cannot make a transition from a positive energy state to a negative one.

Dirac realised that the Pauli exclusion principle would prevent electrons from dropping back to negative energy states if such states were already occupied by negative energy electrons so widespread as to be undetectable. Furthermore, if a negative energy electron was raised to a positive energy state, it would leave behind an unoccupied negative energy state. This unoccupied negative energy state would act like a positively charged electron or a "positron". Thus Dirac, by his faith in the mathematical part of relativistic quantum mechanics and his reluctance to discard what looked like non-physical solutions, predicted the positron. ${ }^{29}$

This story is even more remarkable for the fact that Dirac was trying to reconcile quantum mechanics with special relativity by reworking Schrödinger's wave mechanics in terms of particle waves. This point of view is, as we now know, largely mistaken. The proper context for reconciling special relativity and quantum mechanics is via quantum field theory, and yet the mathematical component of Dirac's theory has survived; indeed it is an important part of modern quantum field theory [Wei 93, 120-121]. So not only did Dirac's equation play a significant role in predicting a novel entity, despite the relevant solutions seeming non-physical, it did so based largely on false assumptions. It is hard to see how a nominalised version of Dirac's theory would have had the same predictive success. ${ }^{30}$

\subsubsection{The Lorentz Transformations}

This final example is similar to the previous one in some ways. We will see how a set of equations known as the Lorentz transformations were part of a paper written in 1904 by H.A. Lorentz based on some fairly strange assumptions, and yet these

\footnotetext{
${ }^{29}$ The positron was subsequently identified in 1932 as an ingredient of cosmic rays.

${ }^{30}$ Mark Steiner discusses this example in a slightly different context in [Ste 89]. See this paper for other excellent examples of the important role mathematics plays in physical theory. See also [Bak 9?] for further discussion on the role mathematics plays in the progress of science.
} 
same transformations became an integral part of Einstein's special relativity a year later, based on entirely different assumptions. What's more, the predictions of the equations have since been experimentally verified. So again we see an example of some mathematical equations surviving the death of the theory that spawned them, thus suggesting that the mathematics is capturing something that the original theory did not.

The "luminiferous ether" was postulated by physicists in the middle of the nineteenth century ${ }^{31}$ as the medium through which Maxwell's electromagnetic radiation must be transmitted, since a wave propagating through a vacuum seemed altogether too strange. Indeed, even Maxwell seemed to support such a theory [Ber 73, 39]. Furthermore it was proposed that this ether may provide the "absolute rest" frame for Newtonian mechanics. Granted these assumptions, it was then reasonable to assume that the earth should be moving relative to the frame of the ether, so we ought to be able to detect an "ether wind" as a result of this motion.

In 1887 Albert Michelson and Edward Morley conducted an ingenious experiment designed to detect the "ether wind". This experiment made use of a piece of equipment known as an interferometer, which consisted of two arms of equal length set at right angles to one another along which two beams of light were "raced". By observing interference patterns (if any) between the two light beams, very small differences in the average velocities (relative to the earth) of the two beams of light could be detected. Indeed, one would expect a difference, since, by elementary physics, the beam of light travelling into the ether wind (and back again) should travel slower than the beam travelling across the ether wind. The fact that no difference was ever detected, despite exacting levels of precision and many repeats of the experiment, was one of the great problems for physicists in the latter part of the nineteenth and the early twentieth century.

One explanation for the failure of the Michelson-Morley experiment to detect any such velocity difference was offered by George FitzGerald in 1892. FitzGerald proposed that the arm of the interferometer travelling into the ether was shortened by exactly the amount required for the two light beams to take the same time for their respective journeys. This seemingly ad hoc idea was given support by Lorentz in his 1895 paper [Lor 85], and in his 1904 paper [Lor 04] he offered an explanation for this phenomenon in terms of his theory of electromagnetic forces. In this latter paper, Lorentz gives a mathematical statement of this shortening as a function of the velocity of the interferometer relative to the "stationary frame of reference" of

\footnotetext{
${ }^{31}$ The concept of the ether, however, goes back at least as far as Descartes.
} 
the ether. The resulting equations are now known as the Lorentz transformations. These four equations state that there will be a contraction of the length of the arm of the interferometer travelling into the ether but no corresponding contraction in either of the two directions perpendicular to this, and so, in particular, there will be no shortening of the length of the other arm. The fourth equation was a time-dilation equation. This stated that a clock in motion runs more slowly than a stationary one. Lorentz, however, insisted that there was only one "true" time and that the "local time" introduced was just a mathematical device to simplify Maxwell's equations for bodies in motion-it had no physical significance [Ber 73, 71-79].

It is now well known that these equations are an integral part of special relativity, although in special relativity they are based on much more reasonable assumptions: namely the principle of relativity ${ }^{32}$ and the principle of constancy ${ }^{33}$ [Ein 05, 37-38]. In fact it might be argued that in Einstein's theory the Lorentz transformations are derived by careful attention to what we mean by length and time, rather than being motivated by an ad hoc defence of the ether. ${ }^{34}$

The point this example illustrates is that whatever merit Lorentz's theory had, and this merit was quite considerable (since his theory predicted the novel phenomenon of the FitzGerald-Lorentz contraction, amongst other things) it was surely largely in the transformations themselves, since presumably the underlying theory was false. It is hard to see how a nominalistic version of this theory would have made such predictions, since the predictive success of the theory came from the fact that the extremely abstract nature of mathematics allowed abstraction away from the false underlying principles. ${ }^{35}$

\footnotetext{
${ }^{32}$ The laws of physics are the same for all inertial reference frames.

${ }^{33}$ The speed of light (in a vacuum) is a constant for all inertial reference frames

${ }^{34}$ I don't mean to denigrate Lorentz's theory; indeed Lorentz and Henri Poincaré very nearly produced the special theory of relativity between them. In fact the renowned physicist Sir Edmund Whittaker thought Einstein's role in the formulation of special relativity was overemphasized when he summarised Einstein's contribution in his 1910 book on the history of the theories of the ether and electricity in the single sentence: "...Einstein published a paper [the 1905 paper] which set forth the relativity theory of Poincaré and Lorentz with some amplifications, and which attracted much attention" [Ber 73, 72]. Needless to say most physicists did not agree with Whittaker's deflationary account of Einstein's contribution!

${ }^{35}$ To put it another way, there are bridge laws between the mathematised Lorentz theory and the nominalised version of the same, but also between the mathematised Lorentz theory and special relativity.
} 


\subsection{Review of Field's Fictionalism}

To sum up then. I have argued that an entity is dispensable to a theory if a better theory can be constructed from the first, in which the entity in question plays no part. Confirmation theory was seen to be important here, in that it provided grounds on which to base decisions about which of two competing theories is the better. Hence confirmation theory plays a crucial role in indispensability decisions. Furthermore, it is around this sense of 'dispensable' that Quine's indispensability argument revolves. It follows then that any critic of the indispensability argument who wishes to deny that mathematics is indispensable to our best physical theories, is obliged not only to give an account of how scientific theories may be constructed without reference to mathematical entities of any kind, but also to show that the resulting theory is preferable to the original.

While I admit that I have remained rather vague about the details of how to compare theories, nevertheless, I have presented a case for accepting that mathematical entities directly contribute towards qualities such as boldness and unificatory power, which we see as properties of good theories. Critics may argue about degrees of unification and boldness and the like, but at the very least they must demonstrate that scientific theory stripped of all its mathematical entities has some degree of virtue comparable to the original theory (if indeed science can be stripped of all its mathematical entities in the first place). It is difficult to see how this could be done given that, in general, we don't understand why it is that mathematics contributes to the virtue of those portions of science that make use of it. In the words of the renowned mathematical physicist Eugene Wigner:

The miracle of the appropriateness of the language of mathematics for the formulation of the laws of physics is a wonderful gift which we neither understand nor deserve. [Wig 60, 14]

It is perhaps being altogether too unreasonable to expect such a "miracle" of the nominalist's formalisation of science as well. This is not to be taken as a fatal blow to nominalist programs such as Hartry Field's. I merely want to point out what is required of them in the light of the indispensability argument, something which it is all too easy to overlook.

Field himself, however, does seem to recognise at least some of the requirements I have outlined in this chapter. For example, he argues that nominalistic theories can be "more illuminating" than their Platonistic counterparts [Fie 80, 44]. He also 
rejects Craigian reaxiomatisations as a way of showing entities to be dispensable because such axiomatisations are unattractive [Fie 80, 4]. ${ }^{36}$ Field recognises that it is not enough to show that mathematical entities are eliminable from empirical science - he is also committed to ensuring that the nominalised theories are attractive theories. Indeed, he goes to some trouble to highlight what he takes to be the virtues of the nominalised theories (apart from their being nominalistic, of course). Their principal virtue is, essentially, that they offer intrinsic explanations for certain facts that are non-arbitrary. ${ }^{37}$

I have two points in response to this. Firstly, I wonder if there isn't just a hint of question begging going on when Field claims that the Hilbert explanation is "intrinsic". For if one thinks, as the indispensabilist does, that numbers are real and intimately (although not causally) involved with the way the world works, it is not clear that explanations of features of space that do not involve numbers are any more intrinsic than electron-free explanations of lightning. The point is that if you think nominalism is correct, then nominalist explanations will seem intrinsic while Platonist ones will not. The Platonist, of course, will not concede this. Hilbert's explanation is indeed an illuminating explanation of a remarkable fact about space, but it is not clear to me that, short of begging the question against the Platonist, it is an intrinsic explanation.

My second point is that while it is clear that this feature of geometric laws is given a more illuminating explanation in Hilbert's theory it must be remembered that it is only with respect to this feature of geometry that Hilbert's theory claims some advantage. Why believe that this illumination is anything more than what happens when one views familiar things from a different viewpoint? For example, the explanation of why the polynomial $X^{2}+1$ has no real roots is seen very clearly by considering the function $f: \mathbb{R} \rightarrow \mathbb{R}$ such that $f(x)=x^{2}+1$ and noticing that the graph of the latter does not intersect the $x$-axis. This explanation is quite enlightening. ${ }^{38}$ It is not, however, any reason for thinking that the geometric interpretation is any more important than the algebraic interpretation, for there are

\footnotetext{
${ }^{36}$ Also see his remarks in [Fie 80 ] about how to choose between Platonism with a compact, recursively enumerable logic and nominalism with a higher-order logic. Here he tells us that "the methodology to employ in making such decisions is a holist one: we should be guided by considerations of simplicity and attractiveness of overall theory" [Fie 80, 97].

${ }^{37}$ For example, Hilbert's axiomatisation of geometry explains why geometric laws formulated in terms of distance are invariant under multiplication by a positive constant. See section 4.1 .2 of this thesis.

${ }^{38}$ Indeed, that this and other such explanations are so enlightening accounts for the importance of Descartes' great insight into the relationship between geometry and algebra.
} 
various other facts that are more readily explained algebraically. ${ }^{39}$ So too with the case in question. Other features of space are given more illuminating explanations in the standard axiomatisation. ${ }^{40}$

Where does all this leave us then? Field accepts that it is not enough to eliminate mathematical entities from our best scientific theories: the resulting theories must be attractive. I have argued that the resulting theories must, in fact, be more attractive (or at least as attractive) - a small point, but worth bearing in mind. In light of this, Field draws attention to a feature of his nominalised theories which he sees as a point in their favour. I have also drawn attention to some desirable features of the Platonistic theories that seem hard to reproduce without mathematical entities. It appears we're left with a rather unsatisfying stand-off. Indeed, it is hard for either side of this debate to do any better at this time. It's difficult enough to compare the virtues of two theories when they are both available for scrutiny, but in this case, the comparison is near impossible, since the nominalist theories in question are not yet available. This fact, along with the worries about whether Field's program can be completed, ${ }^{41}$ suggests that the Quinean indispensabilist is entitled to wait and see how much of science Field's program can nominalise, and how attractive the resulting theories are. I suspect that even if Field's program can be completed for sufficiently large portions of current science, the resulting theories will lack the required degree of virtue for it to be said that mathematical entities have been shown to be dispensable, but this is just a hunch-we must wait and see. In the meantime, I take it that the indispensability argument stands, since the burden of proof in this debate lies with Field and despite his impressive efforts thus far, he has not yet discharged this burden.

Although I am not yet convinced that Field's program will be successful, I have no doubt about the importance of his program. Indeed I, like Field, believe that the correct philosophical stance with regard to the realism/anti-realism debate in mathematics hangs on the outcome of his program. However, not everyone takes this view! In the next chapter I turn to some criticisms of the indispensability argument due to Penelope Maddy which are in many ways more fundamental than Field's. Maddy disagrees that the indispensability argument is a good argument, even if mathematics turns out to be indispensable to science. If she is right about this, then Field's program is irrelevant to whether mathematical objects ought to

\footnotetext{
${ }^{39}$ Such as the fundamental theorem of algebra.

${ }^{40} \mathrm{Such}$ as the triangle inequality.

${ }^{41} \mathrm{Cf}$. [Mal 82] and footnote 13 of this chapter.
} 
be considered real or not [Mad $96 \mathrm{~b}, 67-68] .{ }^{42}$

\footnotetext{
${ }^{42}$ I wish to thank Hartry Field, Peter Menzies, Graham Oppy, Michael Resnik Peter Roeper, Jack Smart, and a referee for Philosophical Studies for their helpful comments on the paper [Col 9?] from which much of this chapter was derived.
} 
90

CHAPTER 4. FIELD'S FICTIONALISM 


\section{Chapter 5}

\section{Maddy's Objections}

In a series of papers spanning the last five years, ${ }^{1}$ one-time mathematical realist Penelope Maddy has presented some serious objections to the indispensability argument. Indeed, so serious are these objections that she has renounced the realism she so enthusiastically argued for in $[\mathrm{Mad} 90] .{ }^{2}$ That realism depended crucially on indispensability arguments. Although her objections to indispensability arguments are largely independent of one another, there is a common thread that runs through each of them. Maddy's arguments draw attention to problems of reconciling the naturalism and confirmational holism required for the Quine/Putnam indispensability argument. In particular, she points out how a holistic view of scientific theories has problems explaining the legitimacy of certain aspects of scientific and mathematical practices, which presumably ought to be legitimate given the high regard for scientific practice that naturalism endorses.

In the first part of this chapter I will outline the three Maddy objections, followed by a discussion of Maddy's conception of the doctrine of naturalism and, in particular, how this conception differs from the Quinean conception. Then I defend the indispensability argument against each of Maddy's objections, taking particular notice of the role naturalism plays in these objections. This is followed by some concluding remarks.

\footnotetext{
${ }^{1}$ See [Mad 92, Mad 95a, Mad 95b, Mad 9?].

${ }^{2}$ She implicitly renounces the set theoretic realism of Realism in Mathematics in many places, but the only explicit renunciation that I'm aware of is found in footnote 30 of [Mad 9?].
} 


\subsection{The Objections}

\subsubsection{The Scientific Fictions Objection}

The first objection to the indispensability argument, and in particular to confirmational holism, is that the actual attitudes of working scientists towards the components of well-confirmed theories vary "from belief to grudging tolerance to outright rejection" [Mad 92, 280]. In 'Taking Naturalism Seriously' [Mad 94] Maddy presents a detailed and concrete example which illustrates these various attitudes. The example is the history of atomic theory from early last century, when the (modern) theory was first introduced, until early this century, when atoms were finally universally accepted as real. The puzzle for the Quinean "is to distinguish between the situation in 1860, when the atom became 'the fundamental unit of chemistry', and that in 1913, when it was accepted as real" [Mad 94, 394]. After all, if the Quinean ontic thesis is correct then scientists ought to have accepted atoms as real once they became indispensable to their theories (presumably around 1860) and yet renowned scientists such as Poincaré and Ostwald remained sceptical of the reality of atoms until as late as 1904.

For Maddy the moral to be drawn from this episode in the history of science is that "the scientist's attitude toward contemporary scientific practice is rarely so simple as uniform belief in some overall theory" [Mad 94, 395]. Furthermore, she claims that "[s]ome philosophers might be tempted to discount this behavior of actual scientists on the grounds that experimental confirmation is enough, but such a move is not open to the naturalist" [Mad 92, 281], presumably because "naturalism counsels us to second the ontological conclusions of natural science" [Mad 95a, 251]. She concludes:

If we remain true to our naturalistic principles, we must allow a distinction to be drawn between parts of a theory that are true and parts that are merely useful. We must even allow that the merely useful parts might in fact be indispensable, in the sense that no equally good theory of the same phenomena does without them. Granting all this, the indispensability of mathematics in well-confirmed scientific theories no longer serves to establish its truth. [Mad 92, 281]

I will not discuss my reply to this or any other of her objections until I present them all, since at least some of my remarks will apply to more than one of them. 


\subsubsection{The Role of Mathematics in Science}

The next problem for indispensability that Maddy suggests follows on from the last. Once one rejects the picture of a scientific theory as a homogeneous unit, the next question concerns whether the mathematical portions of theories fall within the true elements of the confirmed theories. To answer this question Maddy firstly points out that much mathematics is used in theories that make use of hypotheses that are explicitly false, such as the assumption that water is infinitely deep in the analysis of water waves or that matter is continuous in fluid dynamics. Furthermore, she argues that the hypotheses are indispensable to the relevant theory, since it would be unworkable without them, but it would be foolish to argue for the reality of the infinite simply because it appears in our best theory of water waves [Mad 95a, 254].

Next she looks at instances of mathematics appearing in theories not known to contain explicitly false simplifying assumptions and claims that "[s]cientists seem willing to use strong mathematics whenever it is useful or convenient to do so, without regard to the addition of new abstracta to their ontologies, and indeed, even more surprisingly, without regard to the additional physical structure presupposed by that mathematics" [Mad 95a, 255]. In support of this claim she looks at the use of continuum mathematics in physics. It seems the real numbers are used purely for convenience. No regard is given to the addition of uncountably many extra entities (from the rationals, say) or to the seemingly important question of whether space and time (which the reals are frequently used to model) are in fact continuous or even dense. Nor is anyone interested in devising experiments to test the density or continuity of space and time. She concludes that " $[\mathrm{t}]$ his strongly suggests that abstracta and mathematically-induced structural assumptions are not, after all, on an epistemic par with physical hypotheses" [Mad 95a, 256].

\subsubsection{The Mathematical Practice Objection}

Maddy begins this objection by noting what she takes to be an anomaly in Quinean naturalism, namely that it seems to respect the methodology of empirical science but not of mathematics. It seems that, by the indispensability argument, mathematical ontology is legitimised only insofar as it is useful to empirical science. This, claims Maddy, is at odds with actual mathematical practice, where theorems of mathematics are believed because they are proved from the relevant axioms, not because such theorems are useful in applications $[\operatorname{Mad} 92,279]$. Furthermore, she claims 
that such a "simple" indispensability argument leaves too much mathematics unaccounted for. Any mathematics which does not find applications in empirical science is apparently without ontological commitment. Quine himself suggests that we need some unapplied mathematics in order to provide a simplificatory rounding out of the mathematics that is applied but "[m]agnitudes in excess of such demands, e.g. $\beth_{\omega}$ or inaccessible numbers" 3 should be looked upon as "mathematical recreation and without ontological rights" [Qui 86, 400].

Maddy claims that this is a mistake, as it is at odds with Quine's own naturalism. Quine is suggesting we reject some portions of accepted mathematical theory on nonmathematical grounds. Instead she suggests the following modified indispensability argument: ${ }^{5}$

$[T]$ he successful application of mathematics gives us good reason to believe that there are mathematical things. Then, given that mathematical things

${ }^{3} \beth_{\omega}=\bigcup_{\alpha \in \omega} \beth_{\alpha}$, where $\beth_{\alpha}=2^{\beth_{\alpha-1}}, \alpha$ is an ordinal and $\beth_{0}=\aleph_{0}$. See [End 77, 214-215] for further details.

A cardinal number $\kappa$ is said to be inaccessible iff the following conditions hold: (a) $\kappa>\aleph_{0}$ (some texts omit this condition) (b) $\forall \lambda<\kappa 2^{\lambda}<\kappa$ and (c) It is not possible to represent $\kappa$ as the supremum of fewer than $\kappa$ smaller ordinals (i.e. $\kappa$ is regular). For example, $\beth_{\omega}$ satisfies (a) and (b) but not (c). אo satisfies (b) and (c) but obviously not (a). Inaccessible numbers have to be postulated (by large cardinal axioms) in much the same way as the axiom of infinity postulates (a set of cardinality) $\aleph_{0}$.

${ }^{4}$ More recently Quine has refined his position on the higher reaches of set theory and other parts of mathematics which are not, nor are ever likely to be, applicable to natural science. For instance, in his most recent work, From Stimulus to Science, he suggests:

They are couched in the same vocabulary and grammar as applicable mathematics, so we cannot simply dismiss them as gibberish, unless by imposing an absurdly awkward gerrymandering of our grammar. Tolerating them, then, we are faced with the question of their truth or falsehood. Many of these sentences can be dealt with by the laws that hold for applicable mathematics. Cases arise, however (notably the axiom of choice and the continuum hypothesis), that are demonstrably independent of prior theory. It seems natural at this point to follow the same maxim that natural scientists habitually follow in framing new hypotheses, namely, simplicity: economy of structure and ontology. [Qui 95, 56]

A little later, after considering the possibility of declaring such sentences meaningful but neither true nor false, he suggests:

I see nothing for it but to make our peace with this situation. We may simply concede that every statement in our language is true or false, but recognize that in these cases the choice between truth and falsity is indifferent both to our working conceptual apparatus and to nature as reflected in observation categoricals. [Qui 95, 57]

Elsewhere [Qui 90, 94-95] he expresses similar sentiments.

${ }^{5}$ This suggestion was in fact made earlier by Hartry Field [Fie 80, 4-5] but of course he denies that any portion of mathematics is indispensable to science so he had no reason to develop the idea. 
exist, we ask: By what methods can we best determine precisely what mathematical things there are and what properties these things enjoy? To this, our experience to date resoundingly answers: by mathematical methods, the very methods mathematicians use; these methods have effectively produced all of mathematics, including the part so far applied in physical science. [Mad 92, $280]$

This modified indispensability argument and, in particular, the respect it pays to mathematical practice, she finds more in keeping with the spirit, if not the letter, of Quinean naturalism.

She then goes on to consider how this modified indispensability argument squares with mathematical practice. She is particularly interested in some of the independent questions of set theory such as Cantor's famous continuum hypothesis: Does $2^{\aleph_{0}}=\aleph_{1}$ ? and the question of the Lebesgue measurability of $\Sigma_{2}^{1}$ sets. ${ }^{6}$ One aspect of mathematical realism that Maddy finds appealing is that such questions ought to have determinate answers, despite their independence from the usual ZFC axioms. The problem though, for indispensability-motivated mathematical realism, is that it is hard to make sense of what working mathematicians are doing when they try to settle such questions, or so Maddy claims.

For example, in order to settle the question of the Lebesgue measurability of the $\Sigma_{2}^{1}$ sets, two new axioms have been proposed as supplements to the standard ZFC axioms. The two competing axiom candidates are Gödel's axiom of constructibility, $V=L$, and some large cardinal axiom, such as MC (there exists a measurable cardinal). These two candidates both settle the question at hand, but with different answers. MC implies that all $\Sigma_{2}^{1}$ sets are Lebesgue measurable whereas $V=L \mathrm{im}$ plies that there exists a non-Lebesgue measurable $\Sigma_{2}^{1}$ set. The consensus of informed opinion is that $V \neq L$ and that some large cardinal axiom or other is true, ${ }^{7}$ but the reasons for this verdict seem to have nothing to do with applications in physical science. Indeed, much of the appeal of large cardinal axioms is that they are less

\footnotetext{
${ }^{6} \Sigma_{2}^{1}$ sets are part of the projective hierarchy of sets, obtained by repeated operations of projection and complementation on open sets. The $\Sigma_{2}^{1}$ sets, in particular, are obtained from the open sets (denoted $\Sigma_{0}^{1}$ ) by taking complements to obtain the $\Pi_{0}^{1}$ sets, taking projections of these to obtain the $\Sigma_{1}^{1}$ sets, taking complements of these to obtain the $\Pi_{1}^{1}$ sets and finally, taking the projections of these to obtain the $\Sigma_{2}^{1}$ sets. See [Mad 90, chap. 4] (and references contained therein) for further details and an interesting discussion of the history of the question of the Lebesgue measurability of these sets.

${ }^{7}$ There are, of course, some notable supporters of $V=L$ (in particular, Quine [Qui 90, 95] and Devlin [Dev 77]).
} 
restrictive than $V=L$, so to oppose such axioms would be "mathematically counterproductive" [Mad 95a, 265]. These are clearly intra-mathematical arguments that make no appeal to applications.

Furthermore, if indispensability theory is correct, it is quite possible that physical theories would have some bearing on developments in set theory, since they are both part of the same overall theory. For example, Maddy claims that if spacetime is not continuous, as some physicists are suggesting, ${ }^{8}$ this would undermine much of the need for set theory (at least in contexts where it is interpreted literally) beyond cardinalities such as $\aleph_{0}$. Questions about the existence of large cardinals would be harder to answer in the positive if it seemed that indispensability considerations failed to deliver cardinalities as low as $\beth_{1}$. Maddy thus suggests that indispensability-motivated mathematical realism advocates set theorists looking at developments in physics (theories of quantum gravity in particular) in order to tailor set theory to best accord with such developments. ${ }^{9}$ Given that set theorists in general do not do this, a serious revision of mathematical practice is being advocated, and this, claims Maddy, is a violation of naturalism [Mad 92, 289]. She concludes:

In short, legitimate choice of method in the foundations of set theory does not seem to depend on physical facts in the way indispensability theory requires.

[Mad 92, 289]

I now wish to defend the indispensability argument against the three objections I outlined in this section. Before I look at each objection in detail though, it will be useful to examine Maddy's conception of naturalism a little closer, as my reply to her objections depends on a clear understanding of her naturalism. Indeed, I believe that there has been a certain amount of confusion over how naturalism is to be understood in the context of the indispensability argument, and this confusion has allowed her objections to seem more damaging than perhaps they ought.

\subsection{Maddy's Naturalism}

It might be argued that there are two ways in which Maddy's conception of naturalism differs from Quine's. The first she points out herself:

\footnotetext{
${ }^{8}$ For example, Richard Feynman [Fey 65, 166-167] suggests this

${ }^{9} \mathrm{Cf}$. Chihara [Chi 90, 15] for similar sentiments.
} 
On this view [i.e. Quinean naturalism], the philosopher occupies no privileged position from which to critique the practice of natural science; if philosophy conflicts with that practice, it is the philosophy that must give. As a philosopher of mathematics, I extend this compliment to the practice of classical mathematics as well. $[\operatorname{Mad} 97 \mathrm{a}]$

She then remarks in a footnote that "[i]t isn't clear that Quine would approve this extension" [Mad 97a]. The result of this extension is seen in her modification of the Quine/Putnam indispensability argument which I discussed in section 5.1.3 (page 94). Recall that she finds Quine's rejection of quantities such as $\beth_{\omega}$ against the spirit of naturalism, since accepted mathematical practice is rejected on nonmathematical grounds. I shall discuss this departure from Quinean naturalism in more detail when I come to defend the indispensability argument against the mathematical practice objection. At this stage I merely wish to point out that there is a departure and that Maddy recognises this.

The other way in which Maddy's naturalism might be thought to differ from Quinean naturalism is also illustrated in the above quote. It is seen in the move from "the philosopher occupies no privileged position" to "if philosophy conflicts with [scientific] practice, it is the philosophy that must give". Surely the former does not imply the latter. Quinean naturalism tells us that there is no supra-scientific tribunal, whereas Maddy seems to be suggesting that this implies science itself is in a privileged position. That is, the philosopher of science must merely rubber-stamp any scientific practice. Elsewhere she echoes this view of naturalism. For example, in 'Set Theoretic Naturalism' she writes "the [set theory] methodologist's job is to account for set theory as it is practiced, not as some philosophy would have it be" [Mad 96a, 490].

There is much ground between a first philosophy, which Quine rejects, and the rubber-stamp role which Maddy seems to advocate. For instance, there is the position that science and philosophy are continuous with one another and as such there is no high court of appeal. On this view, the philosopher of science has much to contribute to discussions of both scientific methodology and ontological conclusions, as does the scientific community. It may be that you're inclined to give more credence to the views of the scientific community in the eventuality of disagreement between scientists and philosophers, but even this does not imply that it is philosophy that must always give. I take it that this view of science and philosophy as continuous, 
without either having the role of "high court", is in fact the view that Quine intends. It seems that Maddy's interpretation of naturalism represents a significant departure from this view.

Unfortunately things are not that simple, for Maddy also points out that on her view of naturalism "[c]urrent scientific practice need not be taken as gospel, but as a starting point, as prima facie gospel only, subject to ordinary scientific critique" [Mad 97a]. She then goes on to consider the role of the philosopher:

How [...] does the philosophical methodologist differ from any other scientist? If she uses the same methods to speak to the same issues, what need is there for philosophers at all? The answer, I think, is that philosophical methodologists differ from ordinary scientists in training and perspective, not in the evidential standards at their disposal. [Mad 97a]

The view expressed in the above quotations seems at odds with the previous picture of Maddy's naturalism. In particular, the role of the philosopher suggested in the last passage is decidedly different to the powerless bureaucrat rubber-stamping any scientific practice. Indeed, I have no quarrel with Maddy on the account of naturalism suggested in the above passages.

Which view does she take then? Is it always philosophy that must give or can philosophers participate as equals in debates on scientific methodology? Before answering these questions I think it's important to emphasize that Maddy's claim is that naturalism implies that in the event of a dispute between philosophy and scientific practice it is philosophy that must give, not philosophers that must give. She, like Quine, is against first philosophy no matter who the practitioners are, scientists, philosophers or anyone else. She is careful to point out that the naturalistic enterprise must separate the good philosophy (i.e. the philosophy which is continuous with science) from the bad philosophy (i.e. the first philosophy) and that this is a very difficult enterprise [Mad 95a, 261]. So perhaps rather than "philosophy must give" she really just means first philosophy must give. No doubt Quine would agree with the latter but, as I've already suggested, not the former.

Although in Maddy's writings it is not always clear which of the two formulations of naturalism I've been discussing she endorses, I take it that she does in fact endorse the standard Quinean position of rejecting first philosophy (not all philosophy). My evidence for this is in part passages such as the ones above where she is clearly more careful about stating her position, and in part private communication with Maddy on the matter. So Maddy's naturalism departs from Quine's in only the first way 
(i.e. she extends naturalism to endorse the practice of classical mathematics), but we must be careful, for she sometimes writes as though she departs in the second way as well (i.e. to endorse the "philosophy must give" formulation of naturalism). As we shall see in the next section, both these points are important when considering her objections to the indispensability argument.

\subsection{Defending the Indispensability Argument}

In this section I will reconsider the three objections to the indispensability argument raised in section 5.1 .

\subsubsection{The Scientific Fictions Objection Revisited}

Recall that this objection draws attention to the fact that scientists themselves distinguish between the real and the fictional entities in scientific theories. There are two cases to be considered here. The first is the case of scientific fictions that are clearly intended as fictions. I have in mind here such entities as frictionless planes, inertial reference frames, and incompressible fluids. There are a number of reasons for such entities to be taken to be fictional. One reason is that typically the presumed existence of such entities renders inconsistent either the theory in which they occur or another related theory. ${ }^{10}$ Given that consistency is one of the more important virtues of scientific theories, any entity that renders the best available theory inconsistent is unlikely to be indispensable to that theory (no matter how useful it is) because there exists a better theory (i.e. a consistent theory) that does not quantify over the entity in question. ${ }^{11}$

The second case is more problematic. Here we have some entity, such as the mid-nineteenth-century atom, which was indispensable to the best available theory,

\footnotetext{
${ }^{10}$ For Quine's own treatment of such cases (which differs from that presented here) see [Qui 60, 248-251]. Also Jody Azzouni gives a nice account of fictional entities of science in section 2 of [Azz 97].

${ }^{11}$ Strictly speaking the assertion of the existence of a single entity doesn't render the relevant theory inconsistent. It is the conjunction of that sentence and the rest of the theory that is inconsistent; however, we can quite rightly place the blame on a single sentence (or existence statement) in certain circumstances. Consider the example of the frictionless plane. Appeal to frictionless planes simply make the statement of certain laws of mechanics easier, so omitting such appeals makes little difference to the overall theory. On the other hand, to assert the existence of frictionless planes would require a great deal of modification to existing theory to explain how such an entity as a frictionless plane would be possible, given our current understanding of frictional forces. So, to be more precise, and in keeping with what I've said in chapter 4, I should say that frictionless planes are dispensable to the theory of mechanics.
} 
and yet many working scientists of the time treated it instrumentally. Maddy takes this to be a problem for Quinean naturalism, since the naturalistic philosopher of science must "second the ontological conclusions of natural science" [Mad 95a, 251]. Here she writes as though naturalism prohibits any philosophical critique of scientific methodology, but, as we saw in the previous section, this is a mistake; this is not the way Maddy understands naturalism. Once this misconception is cleared up we see that the door is open for a critique of the sceptical scientists from a philosophical perspective located within the scientific enterprise. The naturalistic philosopher can point to what Putnam calls the "intellectual dishonesty" [Put 71, 347] of using atoms, say, in our best chemical theories, then denying the existence of these very same atoms. This is not to say that the philosopher occupies any privileged position in this debate, but neither is $\mathrm{s} /$ he without power. ${ }^{12}$

It may be that scientists such as Poincaré, who were reluctant to believe in the existence of atoms, were being unduly influenced by some non-naturalistic philosophy (such as verificationism). Here the role of the (naturalistic) philosopher of science is clear: try to convince the scientists in question of the benefits of naturalism and of the consequences for the matter at hand. Again I stress that there is no first philosophy in this strategy, just fair interplay of ideas as one would expect in the holistic, naturalistic, Quinean vision of science. ${ }^{13}$

From what I've said so far, it seems that the Quinean must think that those scientists refusing to believe in atoms prior to 1904 were doing something wrong. Maddy obviously disagrees; she thinks that these scientists were right and that something is wrong with the Quinean position. The crux of this objection, then, seems to rest on which way your intuitions go on this and other such episodes in the history of science. I'm inclined to think that the scientists in question were wrong in this case, but I appreciate that many would not share my intuitions here, so let me investigate briefly other possible responses the Quinean might make. ${ }^{14}$

\footnotetext{
${ }^{12}$ One way this response may be blocked is to insist on the "philosophy must give" reading of naturalism discussed in the last section. While I agree that such a reading of naturalism would block the response of this paragraph, it is not Quine's intended reading of naturalism, and the point of the objection is to demonstrate a tension within the Quinean position.

${ }^{13}$ In fact, I think that those scientists who treated the atomic hypothesis instrumentally were adhering to verificationist philosophical principles. One wonders whether a similar incident could occur now in less verificationist times-I suspect not.

${ }^{14}$ It is also worth noting explicitly that since there was substantial disagreement amongst the scientific community about the ontological status of atoms prior to 1904, and since Maddy clearly endorses Ostwald's and Poincaré's scepticism about such atoms, it seems she must think that those scientists who accepted atoms as real were in the wrong. Thus, I am not alone in opposing some portion of the scientific community on this issue. The real question is not: Who is at odds with
} 
One alternative is to deny that atoms were indispensable to science prior to 1904; however, this seems unpromising. Another is to consider the possibility of the Quinean ontic thesis applying only to cases where the theory in question is well accepted amongst the scientific community (i.e. during periods of what Kuhn calls "normal science"). The suggestion is simply that in cases where the best theory is controversial, for whatever reasons, one may suspend judgment on the ontological commitments of the theory. Similarly, one might think that ontological commitment is not an all or nothing affair-we could have degrees of belief in theories and, in particular, to the ontological commitments of those theories. ${ }^{15}$ If this is correct then we have two alternatives: (i) the controversy over atomic theory at the time gives us good reason to think that prior to 1913 chemistry/atomic theory was in a crisis period and thus the Quinean could suspend judgment on the ontological commitments of the theory (indeed, this may be all that Ostwald and Poincaré were doing), (ii) one could argue that given the evidence at the time it would be unwise to give total commitment to either the existence or the non-existence of atoms-some degree of belief strictly between zero and one would be appropriate (again Ostwald and Poincaré's insistence on more evidence could be taken to be nothing more than this). While I won't pursue these two alternatives any further here, they do seem like promising replies to the Maddy objection which avoid charging Ostwald and Poincaré with "intellectual dishonesty".

One other point worth noting, before moving on, is that the Quinean picture of science is not necessarily intended to be in accordance with every episode in the history of science. Presumably science can go wrong, and when it does, it will not accord with the Quinean picture. Recall, from section 2.2, that Quinean naturalism is, in part, a normative doctrine about how we ought to decide our ontological commitments; it is not purely descriptive. This is not to say that Maddy's example of 19 th century atomic theory is a case where science went wrong. On the contrary, I think some scepticism towards novel entities such as atoms is a healthy part of the scientific method.

the scientific community? but rather: Which portion of the scientific community (if any) do you side with?

${ }^{15}$ You might think that a confirmational holist is committed to belief or disbelief in whole theories so that differential degrees of belief in parts of theories is not an option. This is not the case though Even a confirmational holist such as Quine must decide which parts of a disconfirmed theory are to be rejected and which are to be retained. Such decisions are made by appeal to pragmatic considerations such as simplicity. It seems plausible, at least in the case of a disconfirmed theory, that when it is not clear which part of the theory is to be rejected, one may have different degrees of belief in the various parts. 


\subsubsection{The Role of Mathematics in Science Revisited}

Recall that this is the objection that scientists seem willing to use whatever mathematics is required, without regard to ontic commitment. Given my remarks on Maddy's naturalism and my consequent reply to the scientific fictions objections, my reply to this objection is predictable I think. Firstly, I claim that in cases where mathematics is used in blatantly false hypotheses, such as infinitely deep water in physical theories of waves, we need draw no ontological conclusions from the mathematics used, since the theory as a whole is not taken to be literally true. Maddy and I agree thus far! ${ }^{16}$ Furthermore, I suggest that there is no essential difference between these cases and the case of a physicist using a strong mathematical theory which carries with it certain physical assumptions (such as that space-time is continuous). We no more accept that space-time is continuous because of our use of the reals to model it than we believe that our oceans are infinitely deep because this assumption is sometimes necessary when describing waves. The only difference here is that the latter is clearly false while the former is an open question.

What of the mathematics that appears in theories believed to be true? Here Maddy suggests that the naturalistic philosopher must endorse the view of working scientists, which is simply to use whatever mathematics is convenient, without regard for its apparent ontological commitment and, in particular, without affirming the existence of the entities they are using. As in the previous section, I simply deny that naturalistic philosophers must endorse such apparently dishonest behaviour. I am not suggesting that the naturalistic philosopher need be so heavy handed as to attract the charge of "practising first philosophy", but nonetheless such a philosopher is not without the power to enter into debate with such scientists about their alleged metaphysical dishonesty. ${ }^{17}$

Furthermore, it is not clear that this is the attitude working scientists have towards the mathematics they use. In 'Naturalism and Ontology' [Mad 95a] Maddy

\footnotetext{
${ }^{16}$ According to Michael Resnik's "pragmatic indispensability argument" (see section 1.2.3) the truth of mathematics is presupposed when doing science, even when the scientific theory in which it is being used is false (and even if it is known to be false). His argument thus avoids this objection. Although I have some sympathy with this view, for the sake of the present discussion at least, I will take the less controversial line of drawing ontological conclusions only from theories believed to be true.

${ }^{17}$ Certainly the portrayal of the difference between first philosophy and continuous philosophy as a matter of being "heavy handed" or not is a bit of a caricature, but like most caricatures there is some truth in it. First philosophy is unwilling to compromise; continuous philosophy is not. In any case, I suspect that we can do no better than such vague characterisations, although I won't be pursuing the matter here. Whether first philosophy is afoot is determined on a case-by-case basis and by careful attention to the details of the cases. On this Maddy clearly agrees.
} 
cites the example of Richard Feynman's use of real analysis to describe motion, despite his misgivings about the continuity of space and time. ${ }^{18}$ While it is clear that Feynman is using real analysis because it is convenient, it is not clear that he is doing so without regard for the ontological commitment. After all, real analysis is ubiquitous in modern physics, so perhaps Feynman is thinking that whatever ontological load comes with the use of real analysis is already being carried. Then, given that real analysis would be convenient to use in describing motion, there seems no reason not to use it. ${ }^{19}$ Contrast this case with the controversy surrounding the first usage of calculus in the 17 th century.

My claim, then, is that scientists don't worry too much about the ontological commitments of some mathematical theory, if that theory is already widely used (such as in Maddy's Feynman example). On the other hand, when some novel mathematical theory or entities are introduced, it seems that scientists do worry about the mathematics in question. As I've already suggested, the earliest usage of calculus and, in particular, infinitesimals, seems a clear example of this. Another, more recent, example is the introduction of the Dirac delta function to quantum physics.

In order to get around certain problems (such as differentiating a step function) it was necessary to appeal to a "function", $\delta: \mathbb{R} \rightarrow \mathbb{R}$, with the following properties:

$$
\begin{aligned}
\delta(x) & =0, \quad \forall x \neq 0 \\
\int_{-\infty}^{+\infty} \delta(x) d x & =1
\end{aligned}
$$

The delta function, although very useful, is a rather strange entity and its usage naturally attracted much criticism. Even Dirac, who first introduced the function, was not without some concern:

[A]lthough an improper function [i.e. a Dirac delta function] does not itself have a well-defined value, when it comes as a factor in an integrand the integral has a well-defined value. In quantum theory, whenever an improper function appears, it will be something which is to be used ultimately in an integrand. Therefore it should be possible to rewrite the theory in a form in which the improper functions appear all through only in integrands. One could then eliminate the

\footnotetext{
${ }^{18}$ The Feynman work she refers to is [Fey 63].

${ }^{19}$ This might seem implausible, since surely the description of motion was one of the first uses of real analysis, so to represent it as I have here is anachronistic. I agree, but Feynman is presenting the material in an undergraduate physics textbook [Fey 63] as though this were the first time that real analysis had been put to such a use.
} 
improper functions altogether. The use of improper functions thus does not involve any lack of rigour in the theory, but is merely a convenient notation, enabling us to express in a concise form certain relations which we could, if necessary, rewrite in a form not involving improper functions, but only in a cumbersome way which would tend to obscure the argument. [Dir 58, 59]

If, as Maddy claims, physicists are inclined to simply use whatever mathematics is required to get the job done, without regard for ontological commitments, why was Dirac so intent on dispelling doubts about the use of his new "function"?

You might be inclined to think that Dirac's (and other's) concerns ${ }^{20}$ were entirely concerns about rigour and/or consistency. Indeed, you might think that whenever physicists are concerned about the introduction of new mathematics their only concerns are concerns about rigour and/or consistency. In that case, Maddy could argue that, while her claim that physicists will use whatever mathematics is required is not quite correct, nonetheless, concerns about ontology never constitute a reason for concern over the legitimacy of a piece of mathematics. Certainly concerns about rigour and consistency played important roles in the initial controversy surrounding both infinitesimals and the delta function. That much is clear. It is less clear that these were the only concerns. It would be an interesting exercise to try to disentangle the issues of rigour and ontology in such cases. Fortunately this is not required for the task at hand, as there are other cases where the concerns over the introduction of novel mathematical entities are extremely difficult to interpret as being purely about rigour and/or consistency. The first use of the complex numbers to solve quadratic equations by Cardan, around 1545, springs to mind as a case of a consistent theory over which there was considerable debate.

Unlike the cases of infinitesimals and the Dirac delta function, it appears that it was primarily the unusual nature of the entities concerned that worried those making the earliest use of complex number theory. The controversy was over whether the strange new entity $i=\sqrt{-1}$ was a number. Descartes for one thought not, and introduced the term "imaginary" for complex roots of quadratics [Kli 72, 253254]. Others who were suspicious of complex numbers included Newton [Kli 72 , 254] and even Euler, who, in 1768-69, claimed that complex numbers "exist only in imagination" [Kli 72, 594]. In particular, Newton's suspicions were seemingly due

\footnotetext{
${ }^{20}$ Dirac's informal argument justifying the use of the delta function went some way to dispelling those concerns, and certainly the "function" continued to be used, albeit with reservations. The reservations, however, continued until the mathematical theory of distributions was developed to rigorously justify the delta function's usage. As it turns out, the Dirac delta function is not a function at all but a distribution.
} 
to the lack of physical significance of complex roots [Kli 72, 254]; nothing to do with rigour. Let me make it clear, though, that I'm not claiming there were no concerns about rigour in the debate over the use of complex numbers; it's just that such concerns, if they existed, were secondary to what appear to be ontological concerns.

It is also interesting to note in relation to this case that although complex numbers were used in other areas of mathematics and that work on the algebra of complex numbers continued, despite concerns about their use, often proofs appealing to complex numbers were supplemented with proofs that made no such appeals. It wasn't until Gauss's proof of the fundamental theorem of algebra (in 1799), which made essential reference to complex numbers, and until physical applications for complex function theory were developed (also in the latter part of the eighteenth century) that controversy over the usage of complex numbers gradually began to subside [Kli 72, 595]. In both cases applications were important: the former an intra-mathematical application, the latter a physical application.

Whether controversy surrounding the use of novel mathematical entities in physical theories is widespread or not I'm in no position to say, but at least it seems that there are some cases where physicists are genuinely suspicious of new mathematical entities. Furthermore, in some of these cases it seems extremely plausible that the concerns were, at least in part, concerns about ontology. In any case, even if physicists did use whatever mathematics was required, without regard for ontological considerations, this would not imply that the naturalistic philosopher need simply endorse such behaviour, for reasons I have already made clear.

\subsubsection{The Mathematical Practice Objection Revisited}

This objection to indispensability arguments I take to be the most serious. Recall that this objection suggests that a mathematical realism motivated by indispensability is inconsistent with current accepted mathematical practice. Before addressing the main point of the objection though, I wish to say a few words about Maddy's modified indispensability argument (see section 5.1.3, page 94).

I think Maddy is quite right in claiming that (pure) mathematicians are, by and large, not concerned about the applicability of their mathematics, ${ }^{21}$ and that they believe a particular theorem because it has been proved from the axioms, not because it has useful applications. There is still an important question about what this belief amounts to: Does believing a theorem to be true in this context simply

\footnotetext{
${ }^{21}$ Cf. G.H. Hardy's remarks: "It is not possible to justify the life of any genuine professional mathematician on the ground of the 'utility' of his work" [Hard 40, 119-120].
} 
mean that if the relevant axioms were true, then the theorem would be true, or does it mean the much stronger claim that there is ontological commitment to all the entities of the theorem? Let me illustrate with a simple example. If I tell you that Sherlock Holmes is a detective and that all detectives have keen eyes for detail, then you can reasonably infer that Sherlock Holmes has a keen eye for detail. That is, you may conclude that Sherlock Holmes has a keen eye for detail in the first sense (i.e. it's true if the relevant axioms are true), but you may not conclude that Sherlock Holmes has a keen eye for detail in the second sense (i.e. that Sherlock Holmes exists and has a keen eye for detail). I suggest that when mathematicians believe a particular theorem to be true, independent of whether it has applications, they are speaking in the first sense. Mathematicians believe that the theorem follows from the relevant axioms but remain agnostic about the ontological commitments of the theorem (or the axioms). ${ }^{22}$ The ontological questions are answered if and when this particular fragment of mathematical theory finds its way into empirical science.

In fact, it seems quite right that these two questions ought to be separated in such a way and, moreover, that mathematicians should be largely unconcerned with the question of ontological commitment (in their working lives at least). This is no different to other areas of science. Theoretical physicists may investigate various implications of some given theory without any regard for the ontological commitments of that theory-the ontological commitments will come later, if the theory is found to be useful in explaining empirical findings.

Maddy's concerns run a little deeper than this though. She is concerned that the methodology of set theory also depends on how much set theory is required by physics.

Set theorists appeal to various sorts of nondemonstrative arguments in support of their customary axioms, and these logically imply the existence of $\beth_{\omega}$. Inaccessibles are not guaranteed by the axioms, but evidence is cited on their behalf nevertheless. If mathematics is understood purely on the basis of the simple indispensability argument, these mathematical evidential methods no longer count as legitimate supports; what matters is applicability alone. [Mad 92,

\footnotetext{
${ }^{22}$ Michael Resnik has pointed out to me that mathematicians are concerned with ontological commitment to the extent that they want the mathematical theory in question to have a model. But this is just to say that they want their theories to be consistent. Presumably the set of true mathematical theories is properly contained by the set of consistent mathematical theories, so the job of ontology is to decide which of the consistent mathematical theories are true. My claim that mathematicians are agnostic about ontology is simply the claim that they are largely unconcerned with this task.
} 
$278-279]$

She goes on to suggest that such a conclusion is unacceptable, given her endorsement of "a brand of naturalism that includes mathematics" [Mad 92, 279]. Although I have some sympathy with her concern here, such a critique of the simple Quine/Putnam indispensability argument relies explicitly on Maddy's version of naturalism. In particular, it relies on the first departure from Quinean naturalism that I discussed in section 5.2. Furthermore, this objection cannot be sustained given Quinean naturalism.

This is a rather hollow victory for the Quinean though, if Maddy's brand of naturalism is the more plausible. Fortunately this is not the case. Maddy's naturalism, and its respect for the methodology of mathematics, gains much of its appeal by contrasting it with Quinean naturalism, which allegedly pays little or no respect to purely mathematical methodology. But this portrayal of Quinean naturalism is a gross overstatement. The Quinean can agree with Maddy that naturalism demands respect for mathematical methodology, but that this respect is earned by the work mathematics does both within mathematics and, ultimately, in empirical science. Maddy, it seems, is willing to pay respect to the methodology of mathematics for its work in mathematics alone. I have no serious objection to Maddy on this score. Although I'm inclined to prefer the Quinean account, Maddy's is an interesting alternative that deserves attention. My point is simply that Quinean naturalism also legitimates respect for mathematical methodology, and so this cannot be a reason for preferring Maddy's naturalism over Quine's.

As for the charge that the simple indispensability argument leaves too much mathematics unaccounted for (i.e. any mathematics which does not find its way into empirical science), this seems to misrepresent the amount of mathematics that has directly or indirectly found its way into empirical science. On a holistic view of science even the most abstract reach of mathematics is applicable to empirical science so long as it has applications in some other branch of mathematics, which may in turn have applications in some further branch until eventually one of these find applications in empirical science. Indeed, once put this way it is hard to imagine what part of mathematics could possibly be unapplied. ${ }^{23}$

\footnotetext{
${ }^{23}$ Whether these more abstract reaches can be considered indispensable to empirical science is another matter. All I'm claiming here is that these higher reaches are not ignored by the indispensability argument, as Maddy seems to be suggesting.
} 
Still I concede that there may be such areas; perhaps as Quine suggests, inaccessible numbers. ${ }^{24}$ According to the indispensability argument then, these remote reaches of mathematics are without ontological commitment, so again this seems to me to be right. Maddy's alternative of endorsing ontic commitment to all mathematical entities just because they were arrived at by mathematical methods seems misguided. Mathematicians must be free to investigate possible axiom systems, for instance, without being committed to all the resulting entities. There must be room for what Quine calls "mathematical recreation" ${ }^{25}$ for otherwise it starts to look as though the simple act of a mathematician thinking of some entity implies that such entities exist, and such a position, if not outright absurd, faces huge epistemological problems. ${ }^{26}$ In short, I reject Maddy's modified indispensability argument. I think the original Quine/Putnam argument gives a perfectly adequate account of mathematics as practised.

Now to the mathematical practice objection. Maddy begins this objection by claiming that a mathematical realist must agree that there is a fact of the matter about the truth values of such independent hypotheses as the continuum hypothesis and the measurability of $\Sigma_{2}^{1}$ sets. Clearly from what I've said already, I think that for some statements we may refrain from assigning truth values: in particular, to those in areas of mathematics we consider part of mathematical recreation. ${ }^{27}$ This, however, clearly does not apply to the questions that Maddy is interested in. These are questions about sets of real numbers and, as I've mentioned previously, real analysis is ubiquitous in natural science and so, by indispensability theory, has as great a claim to real status as any portion of scientific theory.

So is it correct that a realist about some class of entity should agree that every

\footnotetext{
${ }^{24}$ Even this may be a bad choice of example, for at least some experts think that such numbers are not only the best way of rounding out set theory, but also of rounding out our physical theories. This view is put forward in [Mar 95], for instance.

${ }^{25}$ As Maddy has pointed out to me, there is nothing in her account that explicitly rules out recreational mathematics. The difference between her and Quine on this point is that for Quine recreational mathematics is marked by its isolation from empirical science, whereas for Maddy it is marked by its differing methodology. For example, the investigation of finite models of arithmetic (see [Prie 9?]) will presumably be considered recreational by both Maddy and Quine; by the former because such models are too restrictive and by the latter because they lack the required relationship with empirical science. Thus we see that on Maddy's account, if a mathematician is using accepted methodology (i.e. doing non-recreational mathematics) to investigate some area of abstract mathematics we must interpret the area of mathematics in question realistically. Again it looks as though the act of mathematical investigation implies the existence of some class of mathematical entities.

${ }^{26}$ Jody Azzouni defends a position not unlike this in [Azz 94].

${ }^{27}$ Although Quine seems to prefer the assignment of truth values in such cases (see footnote 4 this chapter), this is mainly to avoid the complications of non-bivalent logics [Qui 95, 57]. The difference is not really important here.
} 
statement about the entities in question is either true or false? It seems not. Many scientific realists would be inclined to dismiss statements about the simultaneous exact locations and momenta of fundamental particles as neither true nor false. Can the mathematical realist take a similar line with regard to the truth of the continuum hypothesis, for instance? Although this is a line that some mathematical realists may be inclined to take, I don't find this approach at all appealing. The difference between the scientific realist refusing to assign truth values to statements about the positions and momenta of fundamental particles and the mathematical realist refusing to assign truth values to any independent statement of mathematics is that in the former case there is a theorem that states exact limitations on the accuracy of measurements of position and momenta (the Heisenberg uncertainty principle); whereas in the latter case it seems that the refusal to assign truth values is merely to avoid the problem at hand and as such is ad hoc.

Adrian Riskin [Ris 94] argues that the mathematical realist need not accept that there is a fact of the matter about new set-theoretic axioms. He argues that it is quite consistent with Platonism to accept all consistent models of set theory as real, just as most mathematical realists are inclined to think of both Abelian and nonAbelian groups as real. Although I agree with him that such a position is viable and has, as Riskin points out, considerable support from mathematical practice, the claim that all consistent mathematical theories are true is unlikely to receive support from indispensability theory. Recall that the indispensabilist is interested in which of the consistent mathematical theories are required by our best scientific theories and hence deserve to be thought of as true (cf. footnote 22). While it is clear that we require both Abelian and non-Abelian groups in order to do science, it is not so clear that more than one set theory is required. In any case, it seems unlikely that we would require every consistent set theory.

So I agree with Maddy that a mathematical realist ought to believe that there is a fact of the matter about answers to independent questions concerning the real numbers (or provide some cogent reason for not doing so). It then seems natural that such a realist should also concede that since $\mathrm{ZFC}$ is not strong enough to answer such questions, there must be some theory that is strong enough, presumably some extension of ZFC. Now Maddy points out:

that this acceptance of the legitimacy of our independent question [Are $\Sigma_{2}^{1}$ sets Lebesgue measurable?] and [...] the legitimacy of its pursuit is not unconditional; it depends on the empirical facts of current science. The resulting 
mathematical beliefs are likewise a posteriori and fallible. [Mad 92, 285]

After considering the implications of the relevant physical theories, namely quantum gravity, in which the possibility that space-time is discrete arises, she concludes:

[S]et theorists should be eagerly awaiting the outcomes of debates over quantum gravity, preparing to tailor the practice of set theory to the nature of the resulting applications of continuum mathematics. But this is not the case; set theorists do not regularly keep an eye on developments in fundamental physics. Furthermore, I doubt that the set-theoretic investigation of independent questions would be much affected even if quantum gravity did end up requiring a new and different account of space-time; set theorists would still want to settle open questions about the mathematical continuum. [Mad 92, 289]

The first thing to say here is that on the version of the indispensability argument that I endorse (i.e. the original Quine/Putnam argument, without Maddy's modification), if there were no use for continuum mathematics anywhere in science (not just as a model of space-time) then a mathematician involved in settling independent questions of real analysis would be pursuing mathematics that has no ontological commitment. That is, $\mathrm{s} /$ he would be participating in mathematical recreation. This is not, however, to denigrate such behaviour. Certainly continuum mathematics is an interesting area of mathematics, independent of its applications. In any case, real analysis would certainly still be required as a useful, though dispensable, approximation in many applications. The mathematician working in real analysis would be in the same boat as a modern physicist working on Newtonian mechanics or vacuum solutions to the Einstein equation.

The crux of this objection, then, is to give an account of why set theorists do not keep a close watch on developments in physics in order to help settle the independent questions of set theory. I suggest that this might simply be a case of division of labour. Set theorists do what they do best-set theory! If developments in other areas of science are to have impact on their discipline then most set theorists will not be in a position to properly assess that impact. This is no different to other areas of science, except for the matter of the scope of mathematics.

Michael Resnik plausibly suggests in a forthcoming paper [Res 97a] that we may construct a rough ranking of the sciences in terms of their scope, in which mathematics is the most global theory since it is presupposed by physics, which in turn is presupposed by chemistry, and so on. Furthermore, this hierarchy imposes certain natural methodological considerations. Anomalies in fairly specialised areas 
of science, such as molecular biology, are best not resolved by making alterations to more global theories, as alterations in the latter will have ramifications in many other areas of science that will not be foreseen by the molecular biologist. This is nothing more than Quine's Maxim of Minimum Mutilation [Qui 90, 14-15] in action.

So we find two reasons for experts not to resolve problems in their own field by proposing changes to another. The first is simply that typically such experts lack the required expertise in the field in which they are proposing the changes. Secondly, even if they were to possess the required expertise, if the other field were a more global theory than their own, they could not possibly know all the ramifications of such alterations in all the theories that depend on that global theory. Even experts in the more global theory are not in a position to assess such ramifications. This means that scientists working in the most global theories such as mathematics and physics are unlikely to need to revise their theories in light of developments in less global theories. The converse, however, is not true. Scientists working in a particular local theory may need to keep an eye on the relevant global theories to make sure that their work is consistent with those theories. ${ }^{28}$

The relevance of all this to the problem at hand is obvious. Set theorists are working in arguably the most global area of the most global theory. They do not expect to have to modify their theories in light of developments in other areas of science. They are not regularly keeping an eye on less global theories, simply because there is usually no need to do so. On the other hand, it seems that this is a place where the philosopher of mathematics can contribute something. The philosopher of mathematics can keep an eye on developments in other areas of science that may be relevant and assist in the assessment of the importance of those developments and of proposed modifications of current mathematical theory in light of those developments. Of course set theorists would still want to settle the open questions of set theory, regardless of developments in physics, but if such developments meant continuum mathematics had no applications, then set theorists working on the continuum hypothesis, say, would be pursuing mathematical recreation.

\footnotetext{
${ }^{28}$ There are, of course, examples where workers in a less global theory continued research in a particular area known to be inconsistent with a relevant global theory. For example, early evolutionary theory required that the earth be much older than pre-atomic physics allowed. That is, pre-atomic physics could not provide a model of the sun emitting energy for the required length of time required for evolution to take place. In this particular case the anomaly was resolved in favour of the biologists, but this does not alter the point that the biologists were painfully aware that evolutionary theory was in tension with the more global theory of physics.
} 


\subsection{Review of Maddy's Objections}

I have considered Maddy's three objections to indispensability arguments and found that in the cases of the objection from scientific fictions (section 5.1.1) and the objection from the role of mathematics in scientific theories (section 5.1.2), much of the force of the objections derived from taking the "philosophy must give" reading of naturalism. This, I argued, was a mistake since neither Quine nor Maddy takes naturalism in this way. On the standard reading of Quinean naturalism, the force of these objections is much reduced, and in both cases more than one solution is possible.

The third of Maddy's objections, the mathematical practice objection (section 5.1.3), was seen to turn on a misconception about Quinean holism. I argued that because a theory is confirmed or disconfirmed as a whole unit does not imply that each fragment of that theory has the same priority, as Maddy seems to suggest. When modification of a theory is required, Quine's Maxim of Minimum Mutilation implores us to modify those areas of the overall theory upon which the least depends. Resnik's global and local distinction was seen to be particularly useful in bringing out this point. The upshot of this is that, given this understanding of holism, once again the Maddy objection cannot be sustained.

Finally, by allowing room for the possibility of mathematical recreation, as does the original Quine/Putnam indispensability argument, we see that mathematicians may pursue research programs that have no direct or indirect application to empirical science and that this accords very well with actual mathematical practice. Furthermore, this feature of indispensability theory is able to explain Maddy's puzzle about why set theorists would want to settle the open questions of set theory, regardless of the applications of such theory. They would be pursuing mathematical recreation.

In closing I should mention one other related issue with which Maddy is interested and which I have not addressed in this chapter. This issue is that of the different methodological consequences of a Quinean naturalist's approach to the independent questions of set theory, as opposed to Maddy's naturalistic approach. She claims that there are significant differences in the type of justification these two approaches would give for the rejection of $V=L$, for instance. Set theoretic naturalism, as Maddy calls her position, is opposed to this axiom on the grounds that it is too restrictive and that it is desirable to have as rich a set theory as possible, whereas the Quinean naturalist must give some reason to think that $V=L$ 
is false. As Maddy rightly points out "desirability (notoriously!) is no guarantee of truth" [Mad 92, 288]. The matter is made more complicated by Quine's own preference for $V=L$ over large cardinal axioms on the grounds of ontological parsimony [Qui 90, 95]. Maddy claims, in effect, that Quinean naturalism delivers up $V=L$ and that this is the wrong answer, or at least it's at odds with general consensus. Indeed Maddy takes this to be a reason to prefer her naturalism over the Quinean variety. $^{29}$

Whether the disagreement over $V=L$ is due to the differing conceptions of naturalism or whether it is due to different weighting of theoretical virtues such as ontological parsimony, unificatory power, elegance, and so on is not clear. Although this topic holds considerable interest, to pursue it here would take us too far afield, so I must, reluctantly, leave a discussion of these issues for another time. The related issue of whether Maddy is right or not about her conception of naturalism being preferable to Quine's is also of considerable interest, but is somewhat orthogonal to the main thrust of her objections to the indispensability argument. The important issue, for present purposes, is whether Quinean naturalism fails to support the indispensability argument, as Maddy has suggested. In this chapter I have shown that this question should be answered in the negative. ${ }^{30}$

\footnotetext{
${ }^{29}$ She takes up this theme in her forthcoming book Naturalism in Mathematics [Mad 97].

${ }^{30}$ I would like to thank Ian Gold, Drew Khlentzos, Penelope Maddy, W.V. Quine and Michael Resnik for valuable conversations on the matters dealt with in this chapter. I'm further indebted to Penelope Maddy, Michael Resnik and a referee for Philosophia Mathematica for their helpful comments on the paper [Col 98b] from which this chapter is largely derived.
} 
114

CHAPTER 5. MADDY'S OBJECTIONS 


\section{Chapter 6}

\section{Further Objections}

It is generally believed that empirical science provides us with propositions which are a posteriori, contingent and revisable in the light of empirical evidence. Mathematical propositions, on the other hand, are generally believed to be a priori, necessary and unrevisable in the light of empirical evidence. But indispensability theory tells us that mathematical knowledge is in the same epistemic boat as empirical knowledge. The tension is clear, and many authors have exploited this tension, in various ways, to undermine the indispensability argument. In this chapter I will defend this argument against such criticisms. In doing so I will be defending the somewhat controversial consequence of the indispensability argument, that mathematical knowledge has an empirical character.

The idea that mathematics is empirical is certainly not new, nor are criticisms of such a position. J.S. Mill, for example, argued for an empiricist account of mathematics in [Mil 43], and in doing so made himself the subject of a sustained attack from Frege. ${ }^{1}$ Mill took numbers to be properties of aggregates and arithmetic to be highly general laws of nature arrived at by observing the behaviour of physical aggregates when adjoined. Amongst Frege's complaints was that this was to confuse an application of arithmetic with arithmetic itself [Dum 91, 59]. In more recent times, though, there has been something of a revival of empirical accounts of mathematics. ${ }^{2}$ Although many of these accounts have much in common and are of considerable interest, I won't discuss them here, since I am primarily concerned with defending Quine's empirical account of mathematics. That is, mathematical

\footnotetext{
${ }^{1}$ Frege's arguments against Mill are found in many places but are particularly evident in his Die Grundlagen der Arithmetik part II, section 23 [Fre 84].

${ }^{2}$ See, for example, [Lak 76] and [Kit 84].
} 
propositions are known a posteriori, because the existence of mathematical objects can only be established by empirical methods-by their indispensable role in our best scientific theories.

In the first section I will address the objection that indispensability theory makes a mystery of the obviousness and clearly a priori character of at least some mathematical knowledge. In the following section I will address an objection from Alan Musgrave that indispensability theory fails to account for the clear difference in modal status between mathematical knowledge and empirical knowledge, the latter being contingent and falsifiable while the former is necessary and unfalsifiable. In the third section I will address Elliott Sober's objection that mathematical theories cannot share the empirical support of our best scientific theories, because mathematics is a common part of all such theories. Sober claims that mathematics is not being tested in the same way as the clearly empirical claims of science, and so it cannot be confirmed by the usual empirical methods. Finally I will consider the question of the contingency of mathematical truth and, in particular, I will discuss the recent debate between Hartry Field, and Crispin Wright and Bob Hale.

\subsection{The Obviousness of Some Mathematical Truth}

Charles Parsons' grievance with indispensability theory is that it doesn't explain the obviousness of so much mathematics.

The empiricist view, even in the subtle and complex form it takes in the work of Professor Quine, seems subject to the objection that it leaves unaccounted for precisely the obviousness of elementary mathematics (and perhaps also of logic). [Par 80, 101]

According to indispensability theory, mathematical statements such as:

There exists an even prime number

or if we formalise this in the obvious way:

$$
(\exists x)((P x \& E x) \& N x)
$$

are true by virtue of their role in successful empirical theories. But it seems Parsons is right that (6.1) is just obviously true. We don't need to do any physics or chemistry before we can see that two is prime. Furthermore, Quine's metaphor of mathematics (and logic) being centrally located in the web of belief makes matters worse, since 
it is presumably the peripheral portions of our theories, (i.e. those closest to the boundary conditions of experience) that are the most obvious.

My response to this objection is simply to deny that any mathematics is obviously true. After all, Hartry Field and others believe that (6.1) is false, so it can't be all that obvious! It is instructive though, I think, to see why these people believe that (6.1) is false. They don't think (6.1) is false because two is odd or because two is composite. They think (6.1) is false because there is no such object as two. They would agree that the following statement is true:

In the story of number theory there exists an even prime number

and Field, at least, would agree to a conditionalised form such as:

If number theory is true then there exists an even prime number or if we formalise this last statement (again in the obvious way) we obtain:

$$
N_{T} \supset(\exists x)((P x \& E x) \& N x) .
$$

Now I claim that (6.2) is obviously true and it is this or something like it ${ }^{3}$ that we confuse when we mistakenly agree with Parsons that (6.1) is obviously true. It simply can't be the case that (6.1) is obviously true, for this would mean that the debate over the reality of numbers that has been raging for thousands of years has an obvious answer-Platonism is correct. As it turns out, I am a Platonist, but I don't think that it is obvious that there are numbers.

That we confuse statements such as (6.1) with some other closely related statement such as (6.2) under certain circumstances is not terribly surprising. Usually when asked questions about elementary number theory we take the context of such questions to be within number theory where the answers are obvious. Asked the same questions in a metaphysics seminar the answers cease to be obvious. Furthermore, this phenomenon is not peculiar to mathematics. It's presumably obvious that the following statement is true:

There is a difference between the political policies of the

Liberals and the Democrats.

It's obviously true in the context of a discussion of Australian politics, but in the context of metaphysics to admit that (6.3) is true is apparently to hold a commitment

\footnotetext{
${ }^{3}$ Although I think it is (6.2) that is being confused with (6.1), nothing I say here depends on this. All that I require is that there is some statement related to (but not identical with) (6.1) that is obviously true. (6.2) certainly seems like a good candidate.
} 
to the ontological category of differences. Perhaps differences, as an ontological category, do exist, but it's far from obvious that they do. Nonetheless, there is surely some reading of (6.3) that is obviously true, and it is this reading, whatever it may be, that we are confusing with (6.3) itself when we pronounce (6.3) obviously true. ${ }^{4}$

I don't wish to labour what is really quite a simple point, but it is important to see that what is taken to be obvious does depend in an important way on context, and the context of the questions that Parsons is concerned with is that of indispensability theory. This places such questions squarely in the realm of metaphysics where the answers are not obvious, not in the realm of mathematics where the answers are obvious.

I should also point out that there is nothing special about the example (6.1). You might think that it was the existential quantifier that committed you to the existence of two in (6.1), and hence the dispute over its truth value, but this would be a mistake. ' $2+2=4$ ' would have done just as well, for surely this, apart from anything else, asserts the existence of two and four (and the addition function and the identity relation for that matter). You might disagree with this Platonistic construal of ' $2+2=4$ '. You might think that it means something like:

When you take two physical objects (of the right sort) and place them next to two other physical objects (of the right sort), you have four physical objects.

or

When I see the marks on the page ' $2+2$ ' I know that it is permissible to replace such marks with the mark ' 4 '.

Such interpretations have existential commitments to physical objects and marks on pages respectively, but none to numbers. Indeed, some such interpretation may well be the correct reading of ' $2+2=4$ ', but it is certainly not obvious that ' $2+2=4$ ' is about marks on a page or physical objects. It is apparently about numbers! Non-Platonistic interpretations of mathematical propositions, apart from any other problems they face, are just not obvious and this is all I require here. I happen to agree with Frege, Quine, Field and others that statements such as ' $2+2=4$ ' are about numbers but nothing hangs on this. My claim is simply that mathematical statements which do not explicitly existentially quantify over mathematical objects, if taken at face value, do, nonetheless, assert that mathematical objects exist. If

\footnotetext{
${ }^{4} \mathrm{I}$ 'm indebted to Frank Jackson for a useful discussion on this.
} 
they are not taken at face value, then it's not obvious what they are about. In either case they are not obviously true.

John Bigelow, in private conversation, has raised a similar concern about the indispensability argument. He points out that mathematics proceeds (apparently) by a priori means and yet, according to indispensability theory, mathematical truths are of the same kind as empirical truths, that is, a posteriori. To use an example of Bigelow's, we don't have to do any empirical investigations to realise that

$$
\sum_{j=1}^{n}(2 j-1)=n^{2}
$$

Bigelow imagines a Greek mathematician (or anyone untrained in modern science for that matter) sitting down and arranging pebbles to prove this. One simply arranges them thus: ${ }^{5}$

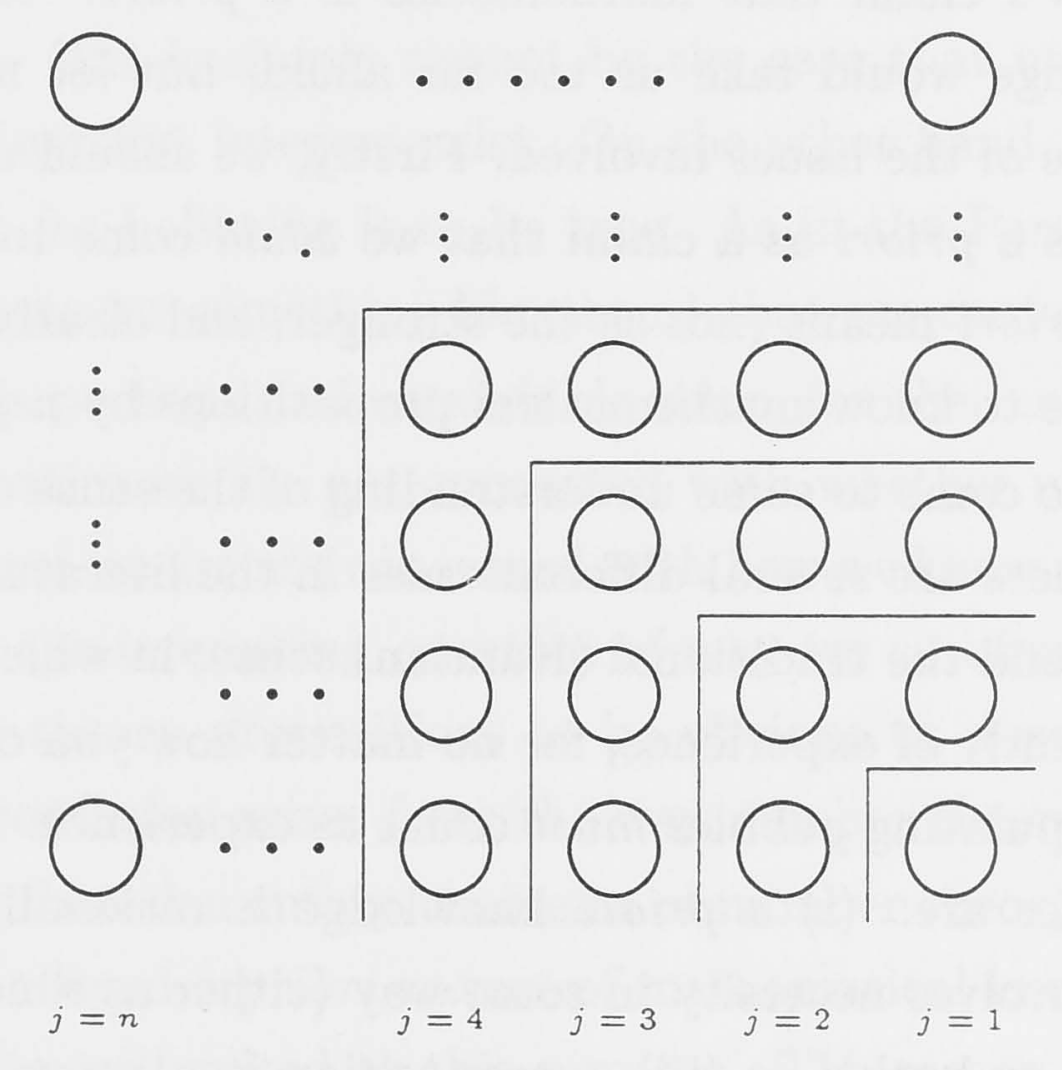

Here each $\Gamma$-shaped collection of pebbles is called a gnomon with the first gnomon $(j=1)$ being the one at the bottom right of the above array (i.e. the gnomon with only one pebble) and the others are numbered from right to left. To see how this arrangement proves equation (6.4) one just needs to see that each gnomon has an odd number of pebbles in it, with gnomon $j=1$ having one pebble and that each successive gnomon to the left has 2 more pebbles than its predecessor. Moreover, the sum of the pebbles in all the gnomons up to $j=n$ is a square array with $n^{2}$ pebbles in it.

\footnotetext{
${ }^{5}$ Bigelow and Pargetter use this example to make a related point in [Big 90, 350-351].
} 
Not only is this obvious, it's a priori. On the Quinean account, mathematical statements such as (6.4) are known to be true by the role they play in our best scientific theories - in other words a posteriori. It's interesting to note that in Bigelow's example (6.4) can be quite easily proved using mathematical induction. This too would be an a priori proof but it would rely on some deeper mathematics which may in turn rely on further mathematics which may eventually depend on a posteriori considerations. Indeed, this is typically what the Quinean is inclined to say about such examples. The Bigelow proof, however, doesn't seem to permit such a move, since it doesn't seem to rely on more mathematics-you just "see it". Apart from some quibbles about whether the pebble arrangement really constitutes a proof (which it does not) and whether it implicitly makes use of mathematical induction (which it does) Bigelow's point is a good one. ${ }^{6}$

Before I can address this worry I need to say something about how we should understand Bigelow's claim that mathematics is a priori. A detailed discussion of a priori knowledge would take us too far afield; but let me make a few brief remarks about some of the issues involved. Firstly, we should understand the claim that mathematics is a priori as a claim that we could come to know mathematical propositions by a priori means (not as the stronger, and clearly false, claim that we do, in general, come to know mathematical propositions by a priori means).

Next, we need to come to some understanding of the sense of a priori Bigelow is invoking here, as there are several different ones in the literature. ${ }^{7}$ For example, he clearly does not intend the traditional (Kantian) sense, in which a priori knowledge is gained independently of experience, for no matter how you cash out 'experience', gazing at and manipulating pebbles must count as experience. Some other accounts of 'a priori' on offer are: (i) a priori knowledge is rationally unrevisable; (ii) a priori knowledge involves necessity in some way (either as a necessary condition, a sufficient condition or both); or (iii) a proposition is a priori if one is justified in believing it once one understands it. I assume Bigelow does not intend (i), since it is entirely possible that his Greek mathematician revises his or her belief in the truth of (6.4) in the light of new evidence (perhaps another pebble arrangement). I

\footnotetext{
${ }^{6}$ It's worth noting the similarity between Bigelow's objection and the objection raised against Quinean naturalism in section 2.3. Recall that the latter objection was that Quinean naturalism didn't respect a methodological difference between philosophy and science: the former proceeding by a priori methods and the latter by a posteriori methods. In discussing that objection I casted doubt on the viability of maintaining the a priori/a posteriori distinction. I stand by my remarks in that section, but here, for the sake of argument, I grant Bigelow that distinction.

${ }^{7} \mathrm{My}$ discussion here follows Albert Casullo's entries 'a priori/a posteriori' and 'a priori knowledge' in [Dan 92, 1-8].
} 
also take it that Bigelow does not intend (ii) since it's hard to see how the pebble arrangement is at all relevant to the necessity of (6.4). Instead, I take it that it's fairly clear that he is using a priori in the third sense. Bigelow's claim then is that the Greek mathematician comes to understand (6.4) via the pebble arrangement and that this is sufficient for him or her to believe (6.4) to be true. ${ }^{8}$

Now to my response to this objection. In short, it is the same as my response to the Parsons objection: I claim that (6.4) is not known a priori. No doubt the claims:

In the story of number theory $\sum_{j=1}^{n}(2 j-1)=n^{2}$

or

$$
N_{T} \supset \sum_{j=1}^{n}(2 j-1)=n^{2}
$$

are known a priori. But it simply cannot be the case that understanding (6.4) is sufficient for believing that integers exist. On the other hand, understanding (6.5) does seem sufficient for believing it to be true. As in the Parsons case, it is these conditional statements (or something like them) that get confused with (6.4).

This confusion is perhaps understandable since, for the most part, when we do elementary mathematics we are not concerned with whether mathematical objects exist or not. A fortiori we are not concerned with how we know whether such objects exist. Still there is the interesting question of why we so often mistakenly believe elementary number theory propositions to be obvious or a priori. One plausible answer is that the confusion arises from the way number theory is learned.

Despite the fact that elementary theorems of number theory are frequently used to demonstrate the alleged a priori nature of mathematical knowledge, I think that number theory is not well suited to this purpose. The reason is that we learn the rudiments of number theory very young and without any concern for ontology. Consequently when we come to consider examples from number theory in the context of deciding whether mathematics is a priori or a posteriori, our intuitions are corrupted by this deeply ingrained, undiscriminating, early training in arithmetic. For example, when you first come across the Peano axioms, usually at university, they

\footnotetext{
${ }^{8}$ James Chase has pointed out to me that Bigelow's problem can be restated using any of these (and perhaps other) senses of a priori. Chase also suggested that this need not concern me too much since, in effect, I deal with the restatements elsewhere. For example, if we take the "rational unrevisability" sense of a priori, the restatement of the Bigelow problem then looks very much like Elliott Sober's objection, which I deal with later in this chapter (section 6.3).
} 
seem obvious, even a priori. To borrow a phrase from Gödel, they "force themselves upon us" [Göd 47, 484]. Try to imagine never having had any training in mathematics up until that time. Would the Peano axioms still force themselves upon you? Fortunately we don't have to answer this question, since there is another area of mathematics, in many ways just as fundamental as number theory (albeit a bit more abstract), which is rarely seen before first year university level. Group theory, I think, is the ideal area to test our intuitions about whether mathematics is a priori or not.

Consider the following very elementary theorem of group theory:

The identity element of a group is unique. ${ }^{9}$

For this theorem to be known a priori the group axioms ${ }^{10}$ need to be a priori. But these, I claim, are clearly, a posteriori. They were developed in order to capture the essential abstract nature of permutations, symmetries, matrix multiplication and many other such examples, so it is necessary for such axioms to capture, for example, non-commutative structures (such as the group $D_{3}$ of symmetries of an equilateral triangle). The non-commutative feature of groups seems very counterintuitive to students when they first encounter group theory. In short, these axioms do not force themselves upon us. Nor does understanding the axioms seem sufficient for believing them.

I have argued that the Bigelow example gains much of its apparent apriority from the confusion between (6.4) and (6.5). This confusion is a result of our unreliable and confused intuitions about rudimentary number theory. When we switch to similar elementary examples in areas such as group theory it is harder to see Bigelow cases having any bite. This is due to the explicit appeal to the group axioms in proofs of elementary group theory results. Such appeal is disguised in the number theory cases by the confusion between what is genuinely a priori and what is just deeply entrenched. ${ }^{11}$

\footnotetext{
${ }^{9}$ Proof: Let $\langle G, *\rangle$ be a group with two identity elements $e$ and $e^{\prime}$. Now let $a$ be any element of $G$ so we have $a * e=a * e^{\prime}=a$. Thus, after multiplying on the left by $a^{-1}$, we have $a^{-1} * a * e=$ $a^{-1} * a * e^{\prime} \Rightarrow e * e=e * e^{\prime} \Rightarrow e=e^{\prime}$.

${ }^{10} \mathrm{~A}$ group $\langle G, *\rangle$ is a set $G$ together with a function $*: G \times G \rightarrow G$ that satisfies the following three axioms: (i) $*$ is associative. i.e. $(a * b) * c=a *(b * c) \forall a, b, c \in G$. (ii) There exists an identity element in $G$, i.e. an element of $G$ (denoted $e$ ) such that for all $x \in G \quad a * e=e * a=a$. (iii) For every $a \in G$ there exists an inverse of $a$, i.e. an element of $G$ (denoted $a^{-1}$ ) such that $a * a^{-1}=a^{-1} * a=e$.

${ }^{11}$ Given that the conditional claims (6.2) and (6.5) are obviously true and can be known a priori, if such claims were sufficient for science then it might be argued that there is no reason to believe in mathematical entities. I agree, but it is far from clear that science can get by with such claims. (The success of Field's program would be required to demonstrate this.)
} 


\subsection{The Unfalsifiability of Mathematical Claims}

Alan Musgrave [Mus 86] has suggested a Popperian worry about the indispensability argument:

Imagine that all the evidence that induces scientists to believe (tentatively) in electrons had turned out differently. Imagine that electron-theory turned out to be wrong and electrons went the way of phlogiston or the heavenly spheres. Popperians think this might happen to any of the theoretical posits of science. But can we imagine natural numbers going the way of phlogiston, can we imagine evidence piling up to the effect that there are no natural numbers? This must be possible, if the indispensability argument is right and natural numbers are a theoretical posit in the same epistemological boat as electrons.

But surely, if natural numbers do exist, they exist of necessity, in all possible worlds. If so, no empirical evidence concerning the nature of the actual world can tell against them. If so, no empirical evidence can tell in favour of them either. The indispensability argument for natural numbers is mistaken. [Mus 86, 90-91]

He then discusses this point in relation to Hartry Field's argument from the conservativeness of mathematics. He asks us to assume that mathematics $(M)$ is both consistent and consistent with any (consistent) scientific theory $(S)$.

[T] hen adding [mathematics] to any scientific theory does not enable us to derive any conclusions about the world that do not follow from the scientific theory alone. (If some conclusion $C$ did follow from $M$ and $S$ which did not follow from $S$ alone, then $M$ would not be consistent with the consistent theory $S \&$ not-C.) Hence the truth or falsity of predictions drawn from a scientific theory with the help of a mathematical theory does not really impinge upon the truth or falsity of the mathematical theory. [Mus 86, 91]

Despite the Popperian trappings in the initial formulation of the problem, there is no mention of Popper or falsification in the second. It thus seems that to criticise the Popperian view of science and the concept of falsification, ${ }^{12}$ in particular, will not deflect this objection. In any case, I will resist this temptation, since I think there are other faults with his argument. I'll consider the two formulations separately because, as we shall see, I believe them to be quite different arguments, albeit with a single underlying intuition. Let me begin with the first formulation.

\footnotetext{
${ }^{12}$ For instance, see [Lak 70a], [Duh 06], and [Qui 51] for criticisms of Popper's falsification principle.
} 
The first thing I wish to question about Musgrave's first formulation is the claim that the indispensability argument implies that one must be able to imagine an experiment that will falsify the proposition that there are natural numbers. This is a rather odd way of suggesting that according to indispensability theory mathematical entities are contingent. As we shall see in section 6.4 I don't think that the indispensability argument forces one to this conclusion, although it is a very natural position for an indispensabilist to adopt. But even granting this, it's not clear to me that we ought to be able to imagine an experiment to falsify the claim that there are numbers. Imagination is a very poor guide to what is possible. For instance, it is possible, we now know, to conduct an experiment to falsify the proposition that it is the earth that rotates and not the celestial sphere. This experiment is, of course, Jean Foucault's famous pendulum experiment. ${ }^{13}$ But to confuse imaginability with possibility is to rob Foucault of any credit for the ingeniousness of the experiment. ${ }^{14}$ In short, even if there is a possible experiment that falsifies some particular proposition, it doesn't follow that we are always able to imagine such an experiment. If this were the case we'd all be great experimental scientists. A fortiori the failure to imagine an experiment to falsify the proposition that there are natural numbers does not imply that there can be no such experiment.

From what I've said so far, it's clear that there is no obligation for the indispensabilist to devise an experiment to test the hypothesis that there exist natural numbers. Nonetheless such an experiment can be conceived. Suppose that Hartry Field has completed the nominalisation of Newtonian mechanics but that he and his successors repeatedly fail to nominalise general relativity. Let's also suppose that this second failure gives us good reason to believe that general relativity cannot be nominalised. From this we conclude that mathematical entities are indispensable to general relativity, but not to Newtonian mechanics. In this setting, then, can we imagine an experiment to test the hypothesis that there are natural numbers? The answer is yes. Not only can we imagine such an experiment, we can perform it. In fact many such experiments have been performed over the last 75 years or so, for any experiment that confirms general relativity over Newtonian mechanics is

\footnotetext{
${ }^{13}$ In 1851 Foucault suspended a $60 \mathrm{~m}$ pendulum from the ceiling of the Pantheon in Paris. The pendulum was set in motion and the plane of oscillation was noted, with care being taken to exclude any disturbing forces. Over time the plane of oscillation changed (with respect to the earth) thus providing dramatic and direct confirmation that it was the earth that rotated and not the celestial sphere. See [Abe 87, 89-91] for further details.

${ }^{14}$ Another example of an apparently unfalsifiable proposition for which empirical confirmation was found is the confirmation of atomic theory by the (correct interpretation) of Brownian motion experiments.
} 
such an experiment. In particular, the 1919 Eddington eclipse experiment is such an experiment. ${ }^{15}$

I take it that Musgrave sees our failure to imagine evidence against the existence of natural numbers to support his claim: "if natural numbers do exist, they exist of necessity" [Mus 86, 91] (for otherwise this important premise is not argued for at all). As we've seen, though, imaginative failure is irrelevant to what is necessary and so the indispensabilist need not accept the idea that numbers exist of necessity. (Indeed the thoroughgoing Quinean would dismiss the whole notion of necessity!) If all this is correct, then Musgrave's argument collapses. If natural numbers do not exist of necessity then there is evidence that will count for or against them. That evidence comes from the confirmation of our best scientific theories.

Now to the second formulation of his objection. Here Musgrave skips over an important detail of Field's conservativeness argument. Field requires that the scientific theory in question $(S)$ is a nominalistic theory, and that the consequences are nominalistic consequences [Fie 80,7-19]. So the conservativeness of mathematics amounts to the claim that no nominalistic claim about the world is a consequence of $M$ and $S$ unless it is a consequence of $S$ alone. Musgrave's statement of this argument is either fallacious or question begging. To see this let $C=M$ and assume $M$ doesn't follow from $S$; then $C$ doesn't follow from $S$ alone, yet it's trivial that $C$ follows from $M$ and $S$. For Field such cases don't arise because $S$ must be a nominalistic theory and $C$ must be nominalist consequences, so we can't have $C=M$. But Musgrave describes the consequences as "about the world" [Mus 86, 91]. That is, either his argument admits the above counterexample or Musgrave takes a proposition being "about the world" to be the same its being nominalistic. The latter begs the question against the mathematical realist who takes mathematical existence claims to be about the world.

Still, let's grant Musgrave a little charity. Let's suppose that what he means by 'about the world' is 'about the physical world'. If this is the case then it does seem reasonable to suppose his argument is the same as Field's conservativeness argument. But this is not an argument against mathematical theories receiving empirical support from the empirical confirmation of our best scientific theories unless such theories can be separated into purely mathematical and purely nominalistic components. To perform such a task is extremely non-trivial. Indeed, Field devotes most of Science Without Numbers [Fie 80] to performing this task on a portion of

\footnotetext{
${ }^{15}$ Colin Cheyne and Charles Pigden use this example in a slightly different context in their paper [Che 96].
} 
Newtonian mechanics. ${ }^{16}$ Whether it can be completed for all of our best scientific theories (in particular, quantum mechanics and general relativity) is controversial. ${ }^{17}$ So even granting Musgrave some charity in his statement of the argument, the argument depends on the successful completion of Hartry Field's nominalisation of science. ${ }^{18}$

It's interesting to reflect upon these two formulations of Musgrave's argument. At least as I've interpreted them, they are quite different arguments. The intuition behind them both, however, is the same and is, I believe, mistaken. That intuition is that mathematics cannot make a difference to empirical findings (because mathematics is necessary in the first formulation, and because it's conservative in the second formulation). This intuition, however, is something the Quinean indispensabilist has long since given up.

Before concluding this section I should address one other statement of the unfalsifiability worry that has been put forward. Charles Parsons writes, in response to the claim that mathematics is empirical:

In one sense mathematics may change as a result [of theory change]: the theory of one type of structure may become more salient, that of another type less so. But no proposition of pure mathematics has been falsified. If we view mathematics in this light, no proposition of Euclidean geometry is falsified by the discovery that physical space is not Euclidean. [Par 83a, 196].

But, as Michael Resnik points out, the theory of Euclidean geometry as a theory of physical space was falsified by general relativity [Res 97]. It remains unfalsified as an abstract theory of Euclidean spaces. This move to the abstract formulation, when a substantial mathematical theory of some physical structure is falsified, is what Resnik refers to as a Euclidean Rescue. The mathematical theory is seen as inappropriate to the given physical situation rather than as a falsified mathematical theory. Indeed, one could perform a similar manouvre on other parts of a theory when that theory is falsified. For example, one might think that the Ptolemy planetary model is unfalsified as an abstract theory (as opposed to a model of the solar system). But there seems little interest in such a rescue of Ptolemic astronomy.

\footnotetext{
${ }^{16}$ In relation to this very point Field says: "It is only relative to the assumption that we could carry out the program of showing mathematical entities to be eliminable that the existence or nonexistence of mathematical objects can be said to be beyond the range of possible evidence" [Fie 93 , 296] (Field's italics).

${ }^{17}$ See, for example, [Mal 82].

${ }^{18} \mathrm{I}$ discussed this matter in chapter 4.
} 
The important difference, then, is not that mathematics is unfalsifiable; it's that we are inclined to perform Euclidean rescues only on mathematical theories. The reason for this, Quine points out, is that the vocabulary of mathematics (and logic) "pervades all branches of science, and consequently their truths and techniques are consequential in all branches of science" [Qui 86, 399]. He continues:

This [...] is why we are disinclined to tamper with logic or mathematics when a failure of prediction shows there is something wrong with our system of the world. We prefer to seek an adequate revision of some more secluded corner of science, where the change would not reverberate so widely through the system. [Qui 86, 399-400]

So, to reiterate, it's not that mathematical theories are unfalsifiable in any absolute sense; it's just that whenever they are part of a falsified package, the mathematical portion is usually salvaged for reasons of minimum mutilation to the web of belief.

\subsection{The Sober Objection}

Elliott Sober's objection to the indispensability argument [Sob 93] is framed from the viewpoint of contrastive empiricism, so it will be necessary to first consider some of the details of this theory in order to evaluate the force of Sober's objection. As will become apparent, though, contrastive empiricism has some difficulties which I'm inclined to think cannot be overcome. This, in turn, robs Sober's objection of much-but not all-of its force. In the final part of this section, then, I will recast the objection without the contrastive empiricism framework and show that this version of the objection also faces significant difficulties.

\subsubsection{Contrastive Empiricism}

As we shall see, contrastive empiricism is best understood as a position between scientific realism and Bas van Fraassen's constructive empiricism. ${ }^{19}$ The central idea of contrastive empiricism is the appeal to the Likelihood Principle as a means of choosing between theories.

Principle 3 (The Likelihood Principle) Observation $O$ favours hypothesis $H_{1}$ over hypothesis $\mathrm{H}_{2}$ iff $\mathrm{P}\left(\mathrm{O} \mid \mathrm{H}_{1}\right)>\mathrm{P}\left(\mathrm{O} \mid \mathrm{H}_{2}\right)$.

\footnotetext{
${ }^{19}$ See [vanF 80] for details of constructive empiricism.
} 
It's clear from principle 3 that the support an hypothesis receives is a relative matter. As Sober puts it:

The Likelihood Principle entails that the degree of support a theory enjoys should be understood relatively, not absolutely. A theory competes with other theories; observations reduce our uncertainty about this competition by discriminating among alternatives. The evidence we have for the theories we accept is evidence that favours those theories over others. [Sob 93, 39]

According to Sober, though, evidence can never favour one theory over all possible competitors since "[o]ur evidence is far less powerful, the range of alternatives that we consider far more modest" [Sob 93, 39].

Another consequence of principle 3 is that some observational data may fail to discriminate between two theories. For instance contrastive empiricism cannot discriminate between standard geological and evolutionary theory, and Philip Henry Gosse's theory that the earth was created about 4,000 years ago with all the fossil records and so on in place. Indeed, Sober's account cannot rule out any cleverly formulated sceptical hypothesis. Furthermore, Sober is reluctant to appeal to simplicity or parsimoniousness as non-observational signs of truth, and so such sceptical problems are taken to be scientifically insoluble. This is one important way in which contrastive empiricism departs from standard scientific realism (and, arguably, standard scientific methodology!).

Although according to contrastive empiricism "science attempts to solve discrimination problems" [Sob 93, 39] and the burden of solving these problems is placed firmly on the observational data, there is no restriction to hypotheses about observables, as in van Fraassen's constructive empiricism [vanF 80].

Contrastive empiricism differs from constructive empiricism in that the former does not limit science to the task of assigning truth values to hypotheses that are strictly about observables. What the hypotheses are about is irrelevant; what matters is that the competing hypotheses make different claims about what we can observe. Put elliptically, the difference between the two empiricisms is that constructive empiricism focuses on propositions, whereas contrastive empiricism focuses on problems. The former position says that science can assign truth values only to propositions of a particular sort; the latter says that science can solve problems only when they have a particular character. [Sob 93, 41] 
Much more could be said about contrastive empiricism, but we have seen enough to motivate Sober's objection to indispensability theory which I discuss in the next section.

\subsubsection{The Objection}

Sober begins by explaining what indispensability amounts to in the setting of contrastive empiricism.

When a scientist considers a set of competing hypotheses, and one of them says that the observations were quite probable, while the other hypotheses say that the observations were immensely improbable, it is natural to conclude that only the first hypothesis makes the observations nonmiraculous. The scientist may be inclined to regard the first hypothesis as indispensable. [Sob 93, 38]

However, Sober thinks that the scientist in question would be in error in regarding the first hypothesis as indispensable. Sober believes that only dispensable statements can enjoy empirical support from observations. To see this he considers a series of competing hypotheses all of which embed some mathematical propositions. Since mathematical statements are a part of every competing hypothesis, the observational outcome does not favour mathematical statements over their competitors. Indeed, according to Sober, it's precisely the lack of competitors that ensures that mathematics cannot enjoy empirical support, since principle 3 favours a particular theory only when it has competitors and the observational data supports the theory in question.

It is clear that Sober takes mathematics to be indispensable in the sense that it is required in all scientific theories, and that some theory is not indispensable if it has a competitor (however poorly supported the competitor is). But as we discovered in section 4.2 , this is not how we ought to understand 'indispensable' in the context of this debate, for otherwise no entity is indispensable. ${ }^{20}$ Instead, 'indispensable' should be taken to mean that the entity in question is an integral part of the best theories, not a part of all theories. ${ }^{21}$ So Sober is quite mistaken when he claims that "the only statements that are tested by observations are statements that are dispensable" [Sob 93, 44]. His mistake is understandable though. Indeed, the choice

\footnotetext{
${ }^{20}$ Because of Craigian reaxiomatisation. See footnote 19, chapter 4 of this thesis for further deatails.

${ }^{21}$ Note that Resnik's pragmatic indispensability argument (see section 1.2.3) revolves around Sober's reading of 'indispensable'.
} 
of the word 'indispensable' is somewhat unfortunate, since it carries an ambiguity between the two senses in question here. This has no bearing on Sober's main point though.

His main objection is that if mathematics is confirmed along with our best empirical hypotheses, there must be mathematics-free competitors (or at least alternative mathematical theories as competitors).

Formulating the indispensability argument in the format specified by the Likelihood Principle shows how unrealistic that argument is. For example, do we really have alternative hypotheses to the hypotheses of arithmetic? If we could make sense of such alternatives, could they be said to confer probabilities on observations that differ from the probabilities entailed by the propositions of arithmetic themselves? I suggest that both these questions deserve negative answers. [Sob 93, 45-46]

It is important to be clear about what Sober is claiming. He is not claiming that indispensability arguments are fatally flawed. He is not unfriendly to the general idea of ontological commitment to the indispensable entities of our best scientific theories. He simply denies that "a mathematical statement inherits the observational support that accrues to the empirically successful scientific theories in which it occurs" [Sob 93, 53]. This is enough, though, to place him at odds with the Quine/Putnam version of the indispensability argument that I'm defending in this thesis.

In reply to this objection, then, I wish to point out firstly that there are alternatives to number theory. Frege showed us how to express most numerical statements required by empirical science without recourse to quantifying over numbers. ${ }^{22}$ Furthermore, depending on how much analysis you think Hartry Field has successfully nominalised, there are alternatives to that also. (At the very least he has shown that there are alternatives to differential calculus. $)^{23}$

I take the crux of Sober's objection then to be the second of his two questions and I agree with him here that the answer to this question deserves a negative answer. I don't think that Field's version of Newtonian mechanics and standard Newtonian mechanics would confer different probabilities on any observational data, but so

\footnotetext{
${ }^{22}$ For example, 'There are two $F \mathrm{~s}$ ' or 'the number of the $F \mathrm{~s}$ is two' is written as: $(\exists x)(\exists y)(((F x \& F y) \& x \neq y) \&(\forall z)(F z \supset(z=x \vee z=y)))$.

${ }^{23}$ This is only considering "sensible" alternatives. There are, presumably, many rather bad theories which do without mathematics. Perhaps most pseudosciences such as astrology and palm reading do without all but the most rudimentary mathematics.
} 
much the worse for contrastive empiricism! The question of which is the better theory will be decided on the grounds of simplicity, elegance and so on-grounds explicitly ruled out by contrastive empiricism. Indispensability theory does not propose to settle all discrimination problems by purely empirical means, so of course it flounders when forced into the straight-jacket of contrastive empiricism.

You might be inclined to think that since a mathematised theory such as Newtonian mechanics and Field's nominalist counterpart have the same empirical consequences, it can't be said that the mathematics receives empirical support. According to this view, the mathematised version is preferred on the a priori grounds of simplicity, elegance and so on, not on empirical grounds. In reply to this, I simply point out that there is nothing special about the mathematical content of theories in this respect. As I've already mentioned, the reason we prefer standard evolutionary theory and geology over Gosse's version of creationism is for the same apparently a priori reasons. It would be a very odd view, however, that denied evolutionary theory and geology received empirical support. Surely the right thing to say here is that evolutionary theory and geology receive both empirical support and support from a priori considerations. I'm inclined to say the same for the mathematical cases. $^{24}$

Another objection to the whole contrastive empiricism approach to theory choice is raised by Geoffrey Hellman and considered by Sober in [Sob 93]. The objection is that often a theory is preferred over alternatives, not because it makes certain (correct) predictions that the other theories assign very low probabilities to, but rather, because it is the only theory to address such phenomena at all. ${ }^{25}$ Sober points out that the relevance of this to the question of the indispensability of mathematics is that presumably "stronger mathematical assumptions facilitate empirical predictions that cannot be obtained from weaker mathematics" [Sob 93, 52]. ${ }^{26}$ If this objection stands, then the central thesis of contrastive empiricism is thrown into conflict with actual scientific practice. For a naturalist this almost amounts to

\footnotetext{
${ }^{24}$ It is perhaps best to speak of the 'scientific justification of theories', where this includes empirical support and support from a priori considerations. This is clearly the sort of support that our best scientific theories receive, so we see that Sober's concentration on purely empirical support skews the whole debate. (Cf. section TROCT of this thesis.) Thanks to Bernard Linsky for a useful discussion on this point.

${ }^{25}$ Hellman gives the example of relativistic physics correctly predicting the relationship between total energy and relativistic mass. In pre-relativistic physics no such relationship is even postulated, indeed, questions about such a relationship cannot even be posed [Hel 9?].

${ }^{26}$ For example, Geoffrey Hellman argues in [Hel 92] that the weaker constructivist mathematics, such as that of the intuitionists (cf. [Hey 31, Dum 75]), will not allow the empirical predictions facilitated by the stronger methods of standard analysis.
} 
a reductio of the position. Indeed, Sober admits that "[i]f this point were correct, it would provide a quite general refutation of contrastive empiricism" [Sob 93, 52]. I believe that Hellman's point is correct, but first let's consider Sober's reply.

Sober's first point is that when scientists are faced with a theory with no relevant competitors, they can contrast the theory in question with its own negation. He considers the example of Newtonian physics correctly predicting the return of Halley's comet, something on which other theories were completely silent. Sober claims, however, that "alternatives to Newtonian theory can be constructed from Newtonian laws themselves" [Sob 93, 52]. For example, Newton's law of universal gravitation: ${ }^{27}$

$$
F=\frac{G m_{1} m_{2}}{r^{2}}
$$

competes with:

$$
F=\frac{G m_{1} m_{2}}{r^{3}}
$$

and

$$
F=\frac{G m_{1} m_{2}}{r^{4}}
$$

and many others. There is no doubt that such alternatives can be constructed and contrasted with Newtonian theory, but as naturalistic philosophers we are not interested in what scientists could do, we are interested in actual scientific practice. ${ }^{28}$

Sober takes this a little further though when he claims that this is standard scientific practice for such cases [Sob 93, 52-53]. He offers no evidence in support of this last claim, and without a thorough investigation of the history of relevant episodes in the history of science it seems quite implausible. It seems extremely unlikely that scientists were interested in debating over whether it should be $r^{2}, r^{3}$ or $r^{4}$ in the law of universal gravitation, as Sober suggests. ${ }^{29}$ The relevant debate would have surely been over retaining the existing theory or adopting Newtonian theory. At the very least, Sober needs to present some evidence to suggest that scientists are inclined to contrast a theory with its own negation when nothing better is on offer. Until such time, I'm inclined to think he is wrong about this.

In his second point in response to Hellman's objection he considers the possibility of "strong" mathematics allowing empirical predictions that cannot be replicated

\footnotetext{
${ }^{27}$ Here $F$ is the gravitational force exerted on two particles of mass $m_{1}$ and $m_{2}$ separated by a distance $r$, and $G$ is the gravitational constant.

${ }^{28}$ This is not to say that a naturalist must agree with all scientific practice, but a naturalist is certainly on shaky ground when forced to propose radical changes to scientific practice. I discuss this matter in more detail in [Col 98a] and in chapter 5 of this thesis.

${ }^{29}$ Not to mention $r^{2.0000000000001}$ or $r^{1.9999999999999}$.
} 
using weaker mathematics. He points out that strong mathematics also allows the formulation of theories that make false predictions, and that this is ignored by the indispensability argument.

It is a striking fact that mathematics allows us to construct theories that make true predictions and that we could not construct such predictively successful theories without mathematics. It is less often noticed that mathematics allows us to construct theories that make false predictions and that we could not construct such predictively unsuccessful theories without mathematics. If the authority of mathematics depended on its empirical track record, both these patterns should matter to us. The fact that we do not doubt the mathematical parts of empirically unsuccessful theories is something we should not forget. Empirical testing does not allow one to ignore the bad news and listen only to the good. [Sob 93, 53]

The first question is: How is this supposed to disarm the Hellman objection? It may be useful at this point to spell out how I take the Hellman strategy to proceed. Hellman's point is that contrastive empiricism does not account for cases where a theory is preferred because it makes predictions that no other theory is able to address one way or another. If this is accepted, then contrastive empiricism as a representation of how theory choice is achieved seems at best only part of the story, and at worst completely misguided. Furthermore, if it is reasonable to prefer some theory because it correctly predicts new phenomena that other theories are silent on, then it is reasonable to accept strong mathematical hypotheses, since theories employing strong mathematics are able to predict just such phenomena.

I take it that Sober's reply runs like this: contrastive empiricism can accommodate the Hellman examples of scientific theories that address new phenomena. This is done by contrasting such theories with their negations. Thus, a general undermining of contrastive empiricism is avoided. This reply, however, seems to allow that strong mathematics is confirmed, because such theories correctly predict empirical phenomena that theories employing weaker mathematics cannot address. So the cost of saving contrastive empiricism from the Hellman objection is that Sober's original point against the empirical confirmation of mathematics now fails. Here is where the second part of Sober's reply is called upon. The point here is simply that the case of strong mathematics is different from that of bold new physical theories in that strong mathematics can also facilitate false predictions that competing theories are silent on. Thus, the mathematics cannot share the credit for 
the successful empirical predictions, since it won't share the blame for unsuccessful empirical predictions. (One admires Sober's sense of justice here, but as we shall see, it is misplaced.)

There are a couple of interesting issues raised by this rejoinder. Firstly, the rejoinder is in the context of a defence of contrastive empiricism and yet it is not an argument for that thesis. Neither is it an argument depending on contrastive empiricism. It seems like a new objection to the use of indispensability arguments to gain conclusions about mathematical entities. What is more, this objection appears to be independent of his contrastive empiricism. Given that few people (including myself) find contrastive empiricism plausible, I take it that this last point is, in many ways, the more substantial part of his objection to indispensability theory and I will discuss it further in the next section.

\subsubsection{A Residual Worry}

So far I've pointed out that I think Sober is quite wrong about scientists contrasting bold new theories with their negations. At the very least he needs to give some evidence to support his claim that they do. ${ }^{30}$ Indeed, it would be interesting to investigate some candidate cases in detail to shed some light on this issue, but fortunately this is not necessary for the present purposes, since even if I grant Sober his first point (that contrastive empiricism can accommodate Hellman's examples of bold new theories) the second part of his reply also runs into trouble.

Sober claims, in effect, that mathematical theories cannot enjoy the confirmation received by theories that make bold new true predictions because the mathematics is not disconfirmed when it is employed by a theory that makes bold new false predictions. I've already noted that this point is stated independently of contrastive empiricism. Indeed, I take this to be a separate worry about the indispensability argument as applied to mathematical entities. Also bear in mind that it is important to his case that there be a difference between mathematical hypotheses and nonmathematical hypotheses in this respect.

This last claim, though, is false. Many non-mathematical hypotheses can be employed by false theories and not be held responsible for the disconfirmation. Hypotheses about electrons (notoriously!) have been employed by many false theories, and yet we are unwilling to blame them for the lack of empirical support for the

\footnotetext{
${ }^{30}$ It is worth pointing out that he must provide evidence that contrasting theories with their negations is a general phenomenon. Even if there are only one or two counterexamples, contrastive empiricism is in trouble.
} 
theories in question. Astrologers refer to the orbits of the planets in grossly false theories about human behaviour and yet we are not about to blame the planets for the lack of empirical support for astrology. It is surely one of the important tasks of scientists to decide which parts of a falsified theory are in need of revision and which are not. Sober would have us throw out the baby with the bathwater it seems.

Hellman points out that this partial asymmetry between confirmation and disconfirmation is a consequence of confirmational holism [Hel 9?]. When a theory is confirmed, the whole theory is confirmed. When it is disconfirmed, it is rarely the fault of every part of the theory, and so the guilty part is to be found and dispensed with. It's analogous to a sensitive computer program. If the program delivers the correct results then every part of the program is believed to be correct. However, if it is not working it is often because of only one small error. The job of the computer programmer (in part) is to seek out the faulty part of the program and correct it. Furthermore, the programmer will resort to wholesale changes to the program only if no other solution presents itself. This is especially evident when one part of the program is working. In such a case the programmer seeks to make a small local change in the defective part of the program. Changing the programming language, for instance, is not such a change.

Now if we return to Sober's charge that mathematics cannot enjoy the credit for confirmation of a theory if it cannot share the blame for disconfirmation, we see that blaming mathematics for the failure of some theory is never going to be a small local change, due to the simple fact that mathematics is used almost everywhere in science. What is more, much of that science is working perfectly well! Blaming the mathematics is like a programmer blaming the language. And similarly claiming that mathematics cannot share the credit is like claiming that the computer language cannot share the credit for the successful program. In some cases it may well be the fault of the mathematics or the language, but it is not a good strategy to start with changes to these.

Furthermore, we see that mathematics is not alone in this respect. Many clearly empirical hypotheses share this feature of apparent immunity from blame for disconfirmation. Michael Resnik points out that conservation principles seem immune from liability for much the same reasons as mathematics. He goes even further to express doubts about whether such principles could be tested at all in the contrastive empiricist framework and "yet we do not want to be forced to deny them empirical content or to hold that the general theories containing them have not been tested experimentally" [Res 95, 168]. Another such empirical hypothesis is the hypothesis 
that space-time is continuous rather than discrete and dense. ${ }^{31}$

To sum up then. I agree with Sober that there is a problem of reconciling contrastive empiricism with indispensability theory, but for the most part this is because of general problems with the former. In particular, contrastive empiricism fails to give an adequate account of a theory being adopted because it correctly predicts phenomena that its competitors are unable to speak to at all. I agree with Hellman here that this looks like the kind of role mathematics plays in theory selection. Strong mathematics allows the formulation of theories that address phenomena on which other theories are completely silent. Sober's rejoinder is that mathematical hypotheses are different from other scientific hypotheses, in that mathematical hypotheses allow false predictions just as readily as the true ones and yet remain blameless for the former. This rejoinder is in effect a new argument against indispensability theory applied to mathematical entities and, what is more, it is independent of the framework of contrastive empiricism. Nevertheless, the rejoinder faces problems of its own. Firstly it seems to misrepresent the type of holism at issue - the holism at issue has an asymmetry between confirmation and disconfirmation built into it. Secondly, it seems clear that mathematics is not alone in its apparent immunity from blame in cases of disconfirmation.

Before closing this section I should mention Sober's claim that despite formulating the objection in the context of contrastive empiricism, the main point can be separated to some extent from that epistemology. However, he does not seem to have the residual worry that I discussed above in mind. He is concerned that you might be inclined to think (as I do) that contrastive empiricism can't be right because it ignores non-empirical criteria such as simplicity (or indeed consistency!). He then suggests that "even proponents of such non-empirical criteria should be able to agree that empirical considerations must be mediated by likelihoods" [Sob 93, 55]. Presumably his objection goes through granting only this, since according to indispensability theory mathematics is empirical. However, I strongly disagree that empirical considerations must be mediated by likelihoods. I take it that the Hellman point concerning theories that make predictions that other theories are silent on provides clear counterexamples to this. So Sober's main objection to indispensability theory fails because it depends crucially on accepting the Likelihood Principle as the only arbiter on empirical matters. The independent residual worry fails because

\footnotetext{
${ }^{31}$ Peter Forrest [For 95] suggests ways of deciding empirically between continuous space-time and discrete space-time with a smallest length (Planck-length, say), but his paper does not address the question of a discrete space-time with no smallest length.
} 
it doesn't take account of the asymmetric character of confirmational holism.

\subsection{Is Mathematics Contingent?}

So far I have argued that according to indispensability theory mathematical knowledge is a posteriori. Does this imply that mathematical entities are contingent? That is, does the indispensability argument give us reason to believe that there are mathematical entities - but that there might not have been? Although I'm inclined to think that mathematics is contingent, it may be that indispensabilists can go either way on this issue. ${ }^{32}$ After all, Kripke [Kri 80] has shown us how we can have a posteriori necessary truth. ${ }^{33}$ I won't pursue this line of thought though. I just wish to point out that there is an alternative to the view that mathematical objects are contingent and that this alternative is, at least at face value, consistent with the view that mathematical knowledge is a posteriori. Instead, I want to consider what is perhaps a more natural position for an indispensabilist to adopt-that mathematical entities exist contingently - and to defend this position against some recent objections from Bob Hale and Crispin Wright.

Hale and Wright in [Hal 92] and [Hal 94] have criticised Hartry Field for his belief in the contingent non-existence of mathematical entities. According to Field, there are no mathematical objects, but there might have been. Hale and Wright, on the other hand, believe that mathematical entities exist necessarily. Thus Hale and Wright take issue with Field on both the non-existence claim and the contingency claim, but here I'll focus on the debate over the latter. Before discussing their objections it will be helpful, I think, to consider briefly why it is that Field is committed to the contingent non-existence of mathematical entities.

As was discussed in chapter 4 , Field relies on the concept of conservativeness in his fictional account of mathematics and this concept is explained in terms of, and indeed entails, consistency. This latter concept, Hale and Wright point out [Hal 92, $112]$, is something that nominalists need to be very careful about, since both the usual syntactic and semantic accounts quantify over abstracta-sequences of sentences in the former and models in the latter. Field of course is aware of this and so

\footnotetext{
${ }^{32}$ Actually Quine is so wary of modality that he is as unlikely to agree to an entity's existence being contingent as he is to it being necessary. Perhaps it would be more accurate (although somewhat confusing) to say that the Quinean indispensabilist believes in mathematical entities whose existence is not necessary.

${ }^{33}$ Kripke takes identity statements, if true, to be necessarily true. However, the truth of such identity statements cannot be found by purely a priori means. Examples include Phosphorus is Hesperus and water is $\mathrm{H}_{2} \mathrm{O}$.
} 
explains consistency in primitively modal terms. Furthermore, on Field's account, the meaning of the 'it is consistent that' operator is not conveyed by definition but by rules of use [Fie 93, 290]. The upshot of all this is that according to Field, a consistent theory is one which is possibly true (in Field's primitive sense of possibility, of course) [Hal 92, 112-113]. This means that if the Peano axioms are consistent, then they are possibly true. Thus, since Field believes that they are in fact false, they must be contingently false (again assuming their consistency).

Field, it seems, has no choice but to accept contingent nominalism. ${ }^{34}$ As I've already mentioned, I think that contingent Platonism ${ }^{35}$ is a rather natural position for the mathematical realist motivated by indispensability considerations to adopt, but that this position is not compulsory. Still I'm inclined to think that contingent Platonism is correct and so I find myself on Field's side in this debate, since, as Field points out, although the Hale and Wright attack is directed mostly against contingent nominalism, "it is clear that if their arguments [...] were correct, they would also undermine 'contingent platonism" " [Fie 93, 285]. So let me discuss what I take to be Hale's and Wright's two main arguments against the contingency of mathematical entities. ${ }^{36}$

\subsubsection{What is the Contingency Contingent on?}

Hale's and Wright's first objection to the contingency of the existence or nonexistence of mathematical objects is that such a contingency would need to be contingent on something.

It is deeply rooted in our ordinary conception of contingency that there ought in general to be explanations of why things which might have been the case are not the case, and of why things are the case which might not have been the case. But in the present kind of instance-the existence of the integers, or real numbers, for example - there is not even a glimmering of how such an explanation might proceed. This seems a strong sign that the notion of contingency would be misapplied in the present context, and hence that there

\footnotetext{
${ }^{34} \mathrm{Th}$ is is the view that it is contingent whether mathematical entities exist or not, and in fact they do not.

${ }^{35}$ This is the view that it is contingent whether mathematical entities exist or not, and in fact they do.

${ }^{36}$ In fact it turns out (rather surprisingly) that the final version of their argument, even if correct, would not work against contingent Platonism. I shall have more to say on this towards the end of section 6.4.2.
} 
is philosophical error in any assumptions that commit Field to so applying it. [Hal 92, 115]

Field's reply to this is to point out that the conception of possibility at issue (for the explanation of consistency) is logical possibility ${ }^{37}$ not conceptual possibility ${ }^{38}$ and that it is a mistake to expect an account of what every logical contingency is contingent on [Fie 89, 43]. As Field points out

there are surely senses of "contingent" in which saying that God's non-existence is contingent would leave us wanting an account of what it is contingent on; for instance, if by calling it contingent we meant that some alternative historical developments might have brought God into existence. But surely granting the consistency of the existence of God (together with the actuality or at least consistency of God's non-existence) is not granting the contingency of God's non-existence in that sense of contingency. I fail to see how the case of numbers is relevantly different. [Fie 93, 291-292]

Nonetheless, Field grants that the existence of mathematical entities is also conceptually contingent [Fie 93, 285] (he just denies that this follows from consideration of conservativeness), and this also seems the most plausible reading of 'contingency' for the mathematical realist persuaded by indispensability theory.

Now to Hale's and Wright's claim that all conceptual contingencies require explanation for their obtaining or not obtaining. Field points out that this claim admits clear counterexamples, besides the mathematical case.

On the question of why there are no numbers, my view is that this is like why is there matter, or why are there no immaterial minds, or why is there no God: you simply can't expect there to be an answer to such basic existence and non-existence questions ('basic' in the sense of: applied to a whole 'ontological category'). (Private correspondence from Field quoted in [Hal 92, 127])

Field is surely correct here. Hale and Wright are also quick to admit this and even provide other examples of contingencies that do not seem to admit explanation, such as: Why is there something rather than nothing? Such examples lead Hale and Wright to refine their principle that all contingencies require explanation. In their most recent paper [Hal 94, 174] they offer the following:

\footnotetext{
${ }^{37}$ A statement is logically possible (for Field) just in case it is not first order logically contradictory.

${ }^{38} \mathrm{~A}$ statement is conceptually possible (roughly) just in case its negation is not true by virtue of its meaning [Fie 93, 292].
} 
Principle 4 It is contingent that $p$ only if either there could be an explanation why $p$ or there is available a priori an explanation why the (putative) fact that $p$ must resist explanation.

The first and most obvious problem is that this principle is extremely ad hoc. The move to requiring a possible explanation rather than an actual explanation I find completely unmotivated.

I agree, however, that something ought to be said about when an explanation for conceptual contingencies should be forthcoming. On the model of explanation offered by Philip Kitcher [Kit 81] (which both Field and myself are partial to) something can be said about such matters, but in many cases it will not be obvious whether to expect an explanation for certain phenomena in advance. This is because, on Kitcher's account, explanation consists in reducing the number of independent phenomena. That is, explanation may be seen as unification. So this account "does not give explainability the kind of default status that Hale and Wright assume" [Fie 93, 295]. Explanation is desirable but not to be expected-indeed one expects that some phenomena must resist explanation.

Although I'm inclined to think that the ad hoc nature of principle 4 is sufficient for its rejection, and consequently the rejection of the objection which rests on it, there are other problems with this objection. Field points out [Fie 93, 294-295] that Hale's and Wright's refined principles (such as principle 4 above) fare little better against some of the non-mathematical counterexamples, such as any truth of the form 'it is a fundamental law of physics that $P$ '. Indeed Hale and Wright concede

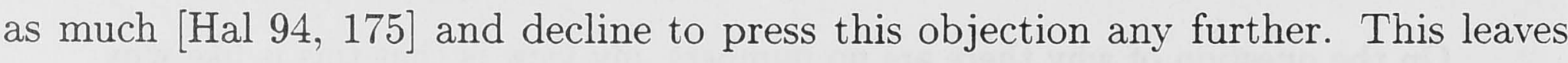
their second argument - the argument from insularity.

\subsubsection{The Argument from Insularity}

Despite declining to press the last objection, Hale and Wright still think that there is something odd about contingent nominalism. This leads them to consider insular contingencies.

Let us call some proposition brute if whether or not it obtains does not depend on anything else. Call a proposition barren if no phenomena depend on whether it obtains or not. Call a proposition absolutely insular if it is both brute and barren. Also note that if Field's program of showing mathematics to be dispensable to science can be carried out, then claims about mathematical entities would appear to be absolutely insular. Absolute insularity, though, is metaphysically dubious. 
This is something that all the participants in this debate agree. The question is what is the correct anti-insularity principle. Hale and Wright [Hal 92, 133-134] suggest the following:

Principle 5 (Hale's and Wright's Anti-insularity Principle) There are no absolutely insular conceptual contingencies.

If Field's program is successful, claims about mathematical entities would be absolutely insular and so by principle 5 it could not be conceptually contingent whether mathematical entities exist or not.

Field, however, thinks that principle 5 is the wrong anti-insularity principle and I agree with him on this. To see this he suggests the following example:

Call something a surdon iff

(A) its existence and state are in no way dependent on the existence and state of anything else; and

(B) the existence and state of nothing else are in any way dependent on the existence and state of it.

This certainly seems to be a conceptually consistent concept; but (A) and (B) guarantee insularity, so principle [5] immediately guarantees the existence of surdons-indeed, the conceptual necessity of their existence. Of course, Hale and Wright accept this conclusion, since they take numbers to be surdons, but even they should balk at the idea that establishing the existence of mathematical entities is as easy as this! [Fie 93, 296-297]

Field concludes that principle 5 can't be right. The correct anti-insularity principle he suggests is:

Principle 6 (Field's Anti-insularity Principle) We should not (at least without very compelling reason) assume the existence of absolutely insular entities.

Thus according to Field, "unless mathematical entities prove indispensable, we ought not to believe in them" [Fie 93, 291].

To this Hale and Wright reply that Field has misconstrued their original argument:

Our objection was not that Field's view of the (putative) fact that there are no numbers infringes an evidently acceptable principle [principle 5], but that it forces an unmotivated decision against an attractive principle. [Hal 94, 180] 
If I may reply on behalf of Field here: surely Field's belief in the contingent existence of mathematical entities is not unmotivated. He has very good theoretical grounds for doing so and he makes this clear in a number of places. Furthermore, he does not rule principle 5 out of play without offering a suitable replacement (principle 6). What is more, his example of surdons demonstrates a point in favour of his own principle 6 over Hale's and Wright's principle $5 .{ }^{39}$

Where does this leave us then? Hale and Wright claim that contingent nominalism would violate principle 5 and since the latter is an attractive metaphysical principle it ought not be forced out of play without good reason. Note that this same objection won't work against the contingent Platonism offered by the indispensability argument, since the reason for believing that there are mathematical entities is precisely because such entities are not absolutely insular. Thus, on this account, the existence of mathematical entities would not violate Hale's and Wright's principle 5. There is something odd about this though. Hale and Wright make it quite clear that it is contingency that is unpalatable. ${ }^{40}$ Why should they be any less concerned by contingent Platonism than contingent nominalism? This is another reason to suggest that principle 5 is not the correct anti-insularity principle. In any case, their final argument against Field, even if it were correct, does not seem to undermine contingent Platonism. I'm inclined to agree with Field, though, that there is nothing problematic about either contingent nominalism or contingent Platonism.

\footnotetext{
${ }^{39}$ Hale and Wright disagree that on their account Platonism is obtained as easily as Field suggests in the above quotation [Hal 94, 181-183]. They claim that conceptual consistency only defeasibly justifies claims of possibility. Thus the conceptual consistency of surdons does not guarantee their necessary existence. Even granting Hale and Wright this, it seems that principle 6 is at least as plausible as principle 5 and so Field is not guilty of an unmotivated rejection of "an attractive principle".

${ }^{40}$ Wright, in an earlier formulation of the concern, states this quite explicitly:

Field has no prospect of an account of what the alleged contingency is contingent on. The world does not, in Field's view, but might have contained numbers. But there is no explanation of why it contains no numbers; and if it had contained numbers, there would have been no explanation of that either. There are no conditions favourable for the emergence of numbers, and no conditions which prevent their emergence. [Wri 88, 465]
} 


\section{Chapter 7}

\section{Conclusion}

So far I've outlined the Quinean indispensability argument and demonstrated its reliance on the doctrines of holism and naturalism (chapter 1 ). I then discussed these doctrines, taking particular care to spell out exactly how each is to be understood, since there is more than one variety of each of these in the literature (chapter 2). In the process of examining naturalism we saw how crucial the Quinean reading is to the indispensability argument, so in chapter 3 I defended the Quinean variety of naturalism against its main competitor. Chapters 4 through 6 were devoted to defending the indispensability argument against some of its critics. Most notable of these are: Field, who denies that mathematics is indispensable to science (chapter 4); Maddy, who questions whether confirmational holism is consistent with Quinean naturalism (chapter 5); and Sober, who questions whether the mathematical content of scientific theories accrue empirical support in the way Quine suggests (section 6.3). If you're convinced by my discussion so far, then you have good cause to accept mathematical entities into your ontology. But you're entitled to ask a little more about the nature of the mathematical entities you're accepting.

We've already seen (from chapter 6) that one consequence of the indispensability argument is that mathematical knowledge has an empirical character. This means that our knowledge of mathematical entities a posteriori. I've also suggested that it is plausible that mathematical knowledge is contingent. There are other questions to be answered though: Does the indispensability argument yield acausal mathematical entities or does it tell us that mathematical entities are causally active after all? Does it tell us that we ought to believe in sets and sets alone, because we can "build" the rest of the mathematical universe out of them? Does it tell us whether sets are sui generis or universals (or something else)? Until now I've been completely silent 
on all such questions, and for good reason-the indispensability argument, as I've presented it, is completely silent on all these issues. I'll elaborate on this claim in the next section.

\subsection{What the Indispensability Argument Doesn't Say}

Although Quine has definite and well-developed views on many of the issues I raised above, it is important to distinguish which of these views depend on the indispensability argument and which depend on other philosophical doctrines. For example, Quine believes that all mathematical entities are sets of some sort or another. Why does he believe this? There's nothing in the indispensability argument that forces this conclusion. Rather, it is a consequence of his extreme Ockhamist tendencies. As Paul Benacerraf puts it (on behalf of Quine) "why demand more than sets, if sets is all you need (sung to the tune of the Beatles' 'All You Need Is Sets')?" [Ben 96, 28]. There's nothing in the indispensability argument that prevents someone with less stark tastes from accepting the whole gamut of mathematical entities. But, the Ockhamist might cry, that's to ignore the well known reductions of numbers to sets, ordered pairs to sets, functions to ordered pairs, and so on. Without the Ockhamist's taste for desert landscapes, ${ }^{1}$ however, it's not clear what these reductions show. It is an interesting fact about mathematics that numbers, for instance, can be modelled in set theory, but this on its own does not tell us that sets and not numbers exist. ${ }^{2}$

As for the question of whether sets (and other mathematical objects) are sui generis or not; again the indispensability argument says nothing. It simply asserts that there are mathematical objects. They might be constituted by more mundane items such as universals and/or relations (as John Bigelow [Big 88], Peter Forrest and David Armstrong [For 87] claim), patterns (as Mike Resnik [Res 97] claims) or the part/whole relation (as David Lewis [Lew 91] claims). Perhaps they are constituted by more exotic items such as possible structures (as Hilary Putnam [Put 67] and Geoffrey Hellman [Hel 89] claim). In short, any (realist) account of mathematical objects is all right by the indispensability argument. It must, of course, be consistent with the view that mathematics has an empirical character (which is the only real requirement of indispensability theory). It is reasonable to

\footnotetext{
${ }^{1}$ This phrase, of course, is due to Quine who says that an "overpopulated universe [...] offends the aesthetic sense of us who have a taste for desert landscapes" [Qui 48, 4].

${ }^{2}$ Paul Benacerraf also makes this point in [Ben 65, 289-290].
} 
assume, however, that this latter requirement can be tacked onto most views easily enough (with the exception of explicitly non-empirical accounts such as Crispin Wright's [Wri 83]).

The question of whether the indispensability argument delivers causal or acausal mathematical entities is a little more complicated. At least some people believe that it delivers causally active entities. Since I think that this is a mistake, I'll discuss the issue in some detail, using a paper by Colin Cheyne and Charles Pigden [Che 96] as my foil. I'll argue that Cheyne and Pigden are mistaken and that the issue of the causal nature of mathematical entities is left open by indispensability theory.

Cheyne and Pigden present their case as a challenge to mathematical Platonism. ${ }^{3}$ The challenge is directed at those Platonists who rely on the Quine/Putnam indispensability argument. As we already know, such Platonists believe the indispensability of mathematics to our best scientific theories gives us good reason to suppose that mathematical entities exist. The Cheyne/Pigden challenge is simply to give an account of how causally inert mathematical entities could be indispensable to science. Failing to meet this challenge, claim Cheyne and Pigden, would place Platonism in a no-win situation: either Hartry Field's nominalisation of science is successful, in which case mathematical entities are dispensable to science, or Field's program fails, in which case mathematical entities may indeed be taken to be indispensable to science but the best explanation for their indispensability is that they are not causally inert as the Platonist assumes. Either way Platonism loses. In what follows I will argue that Platonism is well equipped to meet this challenge-it is not the bad bet Cheyne and Pigden suggest.

Since there is little disagreement that the indispensability argument is without any force if Field's program of nominalising science is successful we need only consider what follows from its failure. Let's suppose, then, that Field's program fails and that this gives us good reason to believe mathematical entities to be indispensable to our best scientific theories. Cheyne and Pigden are unsatisfied with what you might think of as "brute fact" indispensability - the view that we ought to believe in any entity which is indispensable to science and there is no more to be said about it. They would like some account of why it is that mathematical entities are indispensable to science. ${ }^{4}$ This much of their challenge seems quite reasonable, but

\footnotetext{
${ }^{3}$ In fact their challenge is directed at what they call 'standard Platonism'. This is the view that mathematical objects have mind independent existence and that such objects have neither causal powers nor spatio-temporal location. It is to be contrasted with other versions of mathematical realism in which mathematical entities are located in space-time and/or have causal powers.

${ }^{4}$ See also [Bal 96] and [Kit 84, 104-105] for similar worries.
} 
they wish to push the point further.

Why should theories which quantify over certain objects do better than theories which do not? One explanation is ready to hand. If we are genuinely unable to leave those objects out of our best theory of what the world is like [...], then they must be responsible in some way for the world's being the way it is. In other words, their indispensability is explained by the fact that they are causally affecting the world, however indirectly. The indispensability argument may yet be compelling, but it would seem to be a compelling argument for the existence of entities with causal powers. [Che 96, 641]

It is also clear that Cheyne and Pigden think this is not just "one explanation ready to hand" but the only plausible explanation.

I find the above passage puzzling for two reasons. Firstly, the indispensability of a certain entity to some theory says no more than that the entity plays an important role in the theory in question. (This important role we can take to be explanatory.) If you also believe that all explanation is causal explanation (or at least all explanation of events is causal explanation) then it looks as though the entity in question is indeed causally active, but why believe it otherwise? At the very least it seems Cheyne and Pigden have left out a crucial (and controversial) premise from their argument from indispensability to causal activity. ${ }^{5}$ If they do not believe that all explanation is causal then their argument has little force. Furthermore, even if mathematical entities are taken to be causally active, Cheyne and Pigden give little indication of how this explains their indispensability. Presumably not all causally active entities are indispensable to science, ${ }^{6}$ so if some entity's indispensability to a theory is to be explained, more needs to be said than "the entity in question is causally active". In short, Cheyne and Pigden's explanation for the indispensability of mathematical entities is not satisfying as it stands. This takes much of the pressure off the Platonist to come up with a better explanation. ${ }^{7}$

The second difficulty I find with Cheyne and Pigden's argument comes from their failure to say anything about what they take causation to be. This might

\footnotetext{
${ }^{5}$ I discussed and dismissed the view that all explanation is causal in section 3.3 of this thesis.

${ }^{6}$ For example, consider two causally active entities, $a$ and $b$ say, both able to cause some event $e$, although, as a matter of fact, only one entity (suppose it's $a$ ) causes $e$. Now if we have a theory that already makes extensive appeal to $b$ but doesn't seriously entertain the possibility of $a$ existing at all, then it seems reasonable to believe that $b$ is the cause of $e$ and so $a$ is completely dispensable despite its causal activity.

${ }^{7}$ It may well be that the general problem of explaining the indispensability of theoretical entities is a difficult task and the problem of explaining the indispensability of mathematical entities is just a special case of this problem.
} 
seem a harsh criticism since a discussion of causation is a large task which is fairly tangential to their paper. This may be, but their tentative conclusion that the indispensability argument is an argument for causally active mathematical entities seems to revolve around a fairly undiscriminating notion of causation. On a more discriminating notion these same mathematical entities may be causally inert as the (standard) Platonist claims. It looks as though Cheyne and Pigden got their desired conclusion by taking a rather idiosyncratic notion of causation. Allow me to elaborate.

The point Cheyne and Pigden are making is that the world would be different if there were no mathematical entities; therefore mathematical entities are, in some sense at least, partially a cause of the way the world is. This is clearly some form of counterfactual theory of causation and in a later passage, when discussing how Sherlock Holmes might deduce that Moriarty is the murderer by the fact that there are three cigarettes in the ashtray, they give another clue as to what this notion of causation is.

If the number two or the number four were in [three's] place, the effects would differ. What more is needed for it to qualify as an object with causal powers?

[Che 96, 642]

I take it that the "effects" referred to in this passage are not necessarily the effects of the presence of the number three; otherwise it appears that the argument is circular, since whether the number three has causal power is precisely what is at issue here. Instead, I take it that they mean (something like) the future light cones of the world would be different had a different number of cigarettes been present in the ashtray. Does it now follow that the number three is an object with causal powers? If not, then their argument simply doesn't work, but if it does then we see how undiscriminating their notion of causation is. It implies, for instance, that the angle sum of a triangle causes bodies to be accelerated, since if the angle sum of a triangle is $\pi$ radians, the space is (locally) Euclidean and so massive bodies experience no net force; if the angle sum is not $\pi$ radians the space would be non-Euclidean and hence any massive body would be experiencing a net force. Thus, if there were a change in the angle sum of a triangle, the future light cone of the world would be different in that it would contain an accelerated body.

If Cheyne and Pigden take causation to be simple counterfactual dependence, as it seems they do, their conclusion that the indispensability argument gives us good reason to believe in causally active mathematical entities isn't nearly as startling as 
it first seems. Mathematical entities might be causally active, but we're not talking about any common sense of 'causally active' here. (After all, we're not inclined to think that angle sums of triangles can cause bodies to be accelerated.) Furthermore, this simple counterfactual dependence theory of causation doesn't agree with current theories of causation where, for instance, exchanges of energy and/or momentum are involved in causal processes. ${ }^{8}$ Perhaps their conclusion that mathematical entities are causally active could be less misleadingly stated as 'mathematical entities make a difference'. Platonists will have no disagreement with this!

Although the argument from indispensability to causal activity doesn't succeed, the original Cheyne/Pigden challenge still stands. How could causally inert entities play an indispensable role in our best scientific theories? This question is answered by looking at the role such entities play in the relevant theories. The case is no different to that of other theoretical entities. We don't conclude that electrons are causally active simply because they play an indispensable role in our theories of fundamental particles, we conclude that they are causally active because of the role they play in those theories. So too with mathematical entities. We must look at the role they play in our scientific theories. This role is, at least prima facie, not causal. What role do they play then? One fairly plausible possibility considered by Cheyne and Pigden is that "they provide a sort of metaphysical framework" for physics [Che 96, 643]. I agree with Cheyne and Pigden that much work needs to be done on this "framework" theory if it is to be anything more than a metaphor. This, however, is not why Cheyne and Pigden reject the view.

They reject the view that mathematical entities are required as a framework to our best scientific theories because they mistakenly believe that it would have to be a framework "for any possible physics" [Che 96, 643]. They believe that Hartry Field's (partial) nominalisation of Newtonian physics shows that mathematical entities are not indispensable to all possible physics.

The new problem that the platonists face is this: how can a set of necessary beings help explain a contingent set of facts (namely, the facts accounted for by Einsteinian physics), when they would not be needed if the facts were otherwise (i.e., such as to confirm Newtonian physics)? [Che 96, 643]

Clearly their point is very telling against any Platonist who holds the "necessary framework" view, but it completely misses the target of the indispensabilist who denies that mathematical entities are necessary. I've already suggested that this is a

\footnotetext{
${ }^{8}$ For example, see [Ell 90].
} 
very plausible position for an indispensabilist to adopt, and indeed it's the position that I adopt. Such indispensabilists do not claim that mathematical entities are necessary entities or even necessary for any possible physics. I might well concede that were we to live in a Newtonian world we would have no reason to believe in mathematical entities, ${ }^{9}$ but we don't live in such a world! In this, the actual, world we do need mathematical entities to do our physics (or so we are assuming for the purposes of this discussion) and so we cannot dismiss the role mathematical entities play in the actual world because they play no such role in some other possible world. I've already suggested (in section 6.4) that the most plausible view for the Quinean indispensabilist is that mathematical entities are believed to exist contingently and that this is because of the role they play in this world.

It seems that Cheyne and Pigden's claim, that the best explanation for the indispensability of mathematical entities to science is that those entities do, in fact, have causal powers, is unsupported unless they make the further claim that all explanation is causal, and I've already argued (section 3.3) that this is not so. In any case, given the simple counterfactual model of causation they seem to endorse, it looks as though causally active mathematical entities are the least of the unintuitive consequences. I also reject their assessment of the prospects of Platonists meeting their challenge by way of the "framework" role of mathematics. On the contrary, I think once Cheyne and Pigden's confusion over the modal status of the framework is cleared away we see that the view that mathematical entities are contingent has the makings of a very good reply to their challenge. I conclude that the question of the causal capabilities of mathematical entities is left open by indispensability theory. ${ }^{10}$

\subsection{The Benacerraf Challenges}

I noted at the beginning of chapter 1 that mathematical ontology is discussed in the light of three arguments. So far I have been advocating belief in Platonism based on detailed consideration of one of these arguments - the indispensability argument. I'll now take a rather cursory look at the other two arguments-Benacerraf's problems for Platonism - and consider what resources a Quinean indispensabilist has on hand to deal with them. Obviously the details of the replies will depend on the

\footnotetext{
${ }^{9}$ Assuming, of course, that Newtonian physics is suitably nominalised.

${ }^{10}$ I'd like to thank Colin Cheyne, Jack Smart and the referees for The Australasian Journal of Philosophy for their helpful comments on the paper [Col 98b] from which this section was largely derived.
} 
exact details of the account of mathematics and, as I've been at pains to point out, indispensability theory leaves this issue fairly open. Nevertheless, I can at least make a few remarks towards disarming Benacerraf's concerns.

\subsubsection{What Numbers Could Not Be}

In his famous paper 'What Numbers Could Not Be' [Ben 65], Benacerraf set out to undermine the prevailing view that numbers could be reduced to sets. This he did by pointing out that there is no unique reduction of the natural numbers to sets. For instance, we could have $3=\{\emptyset,\{\emptyset\},\{\emptyset,\{\emptyset\}\}\}$ (Zermelo's three) or $3=\{\{\{\emptyset\}\}\}$ (von Neumann's three). Benacerraf then considers possible reasons for preferring one reduction over others, before deciding that there are no such reasons. As Benacerraf puts it:

[I]f the number 3 is really one set rather than another, it must be possible to give some cogent reason for thinking so; for the position that this is an unknowable truth is hardly tenable. But there seems to be little to choose among the accounts. [Ben 65, 284]:

He thus concludes section II of [Ben 65] with the claim that numbers could not be sets at all.

In his essay 'What Mathematical Truth Could Not Be - I' [Ben 96], Benacerraf discusses the arguments of [Ben 65] (amongst other things). Indeed it is very interesting to read Benacerraf's own view on this important paper and its influence on the philosophy of mathematics over the last 31 years. He notes that:

Most of the issues that are raised in the above argument [the argument of sections I and II of [Ben 65]] have received considerable discussion; very few, if any, have been satisfactorily resolved. [Ben 96, 25]

He then considers the weaknesses of the argument. The first is that many people (including Boolos, Dummett and others) feel that the Frege-Russell account or the Frege account of numbers as sets ${ }^{11}$ did not receive due consideration in [Ben 65], and that one of these two is indeed the best candidate for the natural numbers. The second is that, in Benacerraf's words, "no red-blooded realist [...] should accept the bald statement that if there isn't some a priori proof that some particular candidate reduction is the correct one, there can't be a 'correct' one" [Ben 96, 26].

\footnotetext{
${ }^{11}$ On these accounts, the number three, for instance, is identified with the equivalence class of all three-membered sets.
} 
The third weakness concerns what we ought to conclude from a successful reduction of entities of one type to entities of another. The argument of sections I and II of [Ben 65] is primarily directed at reductionists who are not inclined to deny the existence of entities that have been successfully reduced to entities of another kind. Benacerraf likens this type of reduction to packing for a trip to the tropics in which your taking of only one sweater is not to deny the existence of the others you own, it's just that you won't need them. That is, by reducing the natural numbers to some set theoretic structure, all that is to be concluded is that we could do without the numbers since we have an isomorphic copy of them. The numbers are not ruled out of the catalogue of the "furniture of the universe" by such a reduction. Compare this with the thoroughgoing Ockhamist (such as Quine or Field) whose test for inclusion in the aforementioned catalogue is whether the entity in question is necessary for our best theories of the world (considered holistically). The argument of sections I and II of [Ben 65] does not have any impact on this latter position since "the holistic Ockhamite [...] is not beholden to any notion of 'getting it right' that transcends the best theory" [Ben 96, 27]. These three weaknesses suggest three lines of attack on the argument of [Ben 65], and of these Benacerraf has most sympathy with the first: defending the Frege-Russell analysis of natural number as the "right one".

There is, however, another possible reply to the argument of sections I and II of [Ben 65]. Ironically, this reply is motivated by some remarks of Benacerraf's in the third section of [Ben 65]. Benacerraf considers the question of what reductions of numbers to certain sequences of sets is supposed to show. He suggests that such reductions do not show that numbers are really sets after all, any more than the reduction of the natural numbers to certain sequences of oranges shows that natural numbers are really oranges. Benacerraf then combines this move with other (structuralist) assumptions to conclude that numbers cannot be objects at all. (I'll discuss this argument shortly.) I too have considerable sympathy with resisting the view that numbers are sets, but I wish to point out that this view can be used to motivate a quite different conclusion to that which Benacerraf draws. If one denies that the natural numbers are sets then they may plausibly be sui generis objects. Thus one can admit that natural numbers can be modelled in set theory, but that this gives no reason to believe that numbers are sets. Benacerraf's indeterminacy argument has no force against such a position.

In the third section of [Ben 65] Benacerraf pushes the point a little further and argues for the conclusion that "not only could numbers not be sets, they couldn't 
even be numbers" [Ben 65, 23]. This he argues from two assumptions: (i) the structuralist premise that arithmetic is the science of progressions (not a particular progression, but progressions in general), and (ii) the claim that no (particular) system of objects could satisfy (i). The thought is that arithmetic is only concerned with the structural properties of the natural numbers. ${ }^{12}$ Then Benacerraf considers what sorts of objects could have only structural properties and no intrinsic properties. ${ }^{13} \mathrm{He}$ (rather tentatively) ${ }^{14}$ concludes that there can be no such objects because he believes that all legitimate objects must have some non-structural properties.

In response to this argument I wish to make a couple of points. Firstly, it is clear that this argument only has force against structuralists. That is, one could resist the argument by rejecting the structuralist premise that there is nothing more to being the natural number $n$ than being the $n$th member of a sequence of objects. But even accepting structuralism, there are at least two alternatives available. One is to deny (ii), that is, to assert the possibility of objects that have only structural properties. Such entities would be rather strange and unlike any of the usual entities we come across in our daily lives, but we already know that numbers are unlike the more mundane entities we encounter, so such an appeal to weirdness has little force. I don't wish to endorse such a view nor do I wish to argue for it in any detail. I'm merely flagging it as a possible structuralist response.

The other structuralist alternative to the argument of section III of [Ben 65] is to take a more radical structuralist position. On this view, the objects of mathematics are not the individual entities but, rather, the structures taken as wholes. So, for example, the objects of arithmetic are not the individual numbers, but the whole structure that is the natural-number $\omega$-sequence-the numbers themselves are merely positions in this structure. Again, I don't wish to defend this view, I just offer it as a possible solution. ${ }^{15}$

\footnotetext{
${ }^{12}$ It should also be pointed out that this structuralist assumption provides a rather good reply to the arguments of the first two sections of [Ben 65]. According to structuralism, questions that draw our attention to the differences between any two set-theoretic reductions of the natural numbers are ruled out. For instance, the question 'Is $1 \in 3$ ?' (which, without the structuralist premise, would be answered in the affirmative on Zermelo's account and in the negative on von Neumann's) is ruled out as a non-structural question since it's a question about the intrinsic properties of the number three. (Note that this is different to the solution I suggested on page 151.)

${ }^{13}$ There are many different accounts of intrinsic properties. My reference here is simply to nonrelational properties.

${ }^{14}$ Benacerraf points out in [Ben 96] that section III of [Ben 65] has been much misunderstood. The conclusions of section III were being advanced with much less confidence than those of the previous two sections, as they depend on the rather controversial premises (i) and (ii).

${ }^{15}$ See Michael Resnik's recent book Mathematics as a Science of Patterns [Res 97] for a defence of a view along these lines.
} 
There are, it seems, a variety of options available to the indispensabilist for both the indeterminacy argument of sections I and II and for the more sweeping claims of section III of [Ben 65]. Indeed, numbers could be a variety of things (including sui generis entities) and the indispensability argument doesn't legislate against many of these at all.

\subsubsection{Naturalising Mathematical Epistemology}

In 'Mathematical Truth' [Ben 73] Benacerraf challenges philosophers of mathematics to: (i) naturalise mathematical epistemology, and (ii) produce a semantics for mathematics that meshes with that of the rest of language. On a Platonist account of mathematics the second challenge is met easily since a proposition such as 'there exists a prime number greater than 3 ' is made true by the existence of the number 5 (amongst others), just as 'there exists a city larger than Melbourne' is made true by the existence of the city New York (amongst others). The problem for Platonism, however, is to provide a naturalised account of mathematical epistemology. Benacerraf also shows how various anti-realist views of mathematical entities meet the first challenge but not the second. However, it is the challenge to Platonism that [Ben 73] is best remembered for.

Benacerraf explicitly invokes the causal theory of knowledge as an ally in setting up the epistemological problem for Platonism.

I favor a causal account of knowledge on which for $X$ to know that $S$ is true requires some causal relation to obtain between $X$ and the referents of the names, predicates, and quantifiers of $S$. I believe in addition in a causal theory of reference, thus making the link to my saying knowingly that $S$ doubly causal.

[Ben 73, 412]

It is not surprising then that acausal mathematical entities have trouble on such an account. They can neither be referred to nor known about! Since 1973, when 'Mathematical Truth' was first published, ${ }^{16}$ causal theories of knowledge have fallen on hard times. ${ }^{17}$ I won't rehearse the arguments against causal theories here (although I did discuss some of them in chapter 3 of this thesis). ${ }^{18}$ It is sufficient to

\footnotetext{
${ }^{16}$ Although this paper was fairly widely known and circulated as early as 1968 [Ben 96, 10].

${ }^{17}$ See [Sho 83] for a detailed account of their demise. Ironically one of the more important blows to causal theories came in 1973-the same year as 'Mathematical Truth' was published-with Mark Steiner's 'Platonism and the Causal Theory of Knowledge' [Ste 73]. See also Steiner's Mathematical Knowledge [Ste 75], especially chapter 4.

${ }^{18}$ Perhaps their biggest failing is that they do not solve the Gettier problems that motivated
} 
note that Benacerraf's challenge to Platonism explicitly depends on a now largely discredited epistemology. ${ }^{19}$ That ought to be the end of the matter, but alas it's not. Despite the demise of the causal theory of knowledge, there remains considerable unease amongst many philosophers over the prospects of a naturalised epistemology for mathematics. For instance, W.H. Hart writes:

$[\mathrm{I}] \mathrm{t}$ is a crime against the intellect to try to mask the problem of naturalizing the epistemology of mathematics with philosophical razzle-dazzle. Superficial worries about the intellectual hygiene of causal theories of knowledge are irrelevant to and misleading from this problem, for the problem is not so much about causality as about the very possibility of natural knowledge of abstract objects. [Hart 77]

What is it about Benacerraf's problem that allows it to outlive its major premise (and perhaps both its explicit premises ${ }^{20}$ )? As Maddy suggests, "there is a certain undeniable fascination to a problem so resilient!" [Mad 96b, 64].

Hartry Field suggests that Benacerraf's problem for Platonism may be restated as a problem of explaining the reliability of our mathematical beliefs.

Benacerraf's challenge - or at least, the challenge which his paper suggests to me - is to provide an account of the mechanisms that explain how our beliefs about these remote entities can so well reflect the facts about them. The idea is that if it appears in principle impossible to explain this, then that tends to undermine the belief in mathematical entities, despite whatever reasons we might have for believing in them. Of course, the reasons for believing in mathematical entities (in particular, the indispensability arguments) still need to be addressed, but the role of the Benacerrafian challenge (as I see it) is to raise the cost of thinking that the postulation of mathematical entities is a proper solution, and thereby increase the motivation for showing that mathematics is not really indispensable after all. [Fie 89, 26]

This formulation of Benacerraf's problem does not depend on the causal theory of knowledge. Indeed, as Field is quick to point out, it doesn't depend on any theory of knowledge [Fie 88, 232-233]. I thus take Field's formulation to be a vast

${ }^{19}$ Penelope Maddy suggests that Benacerraf's challenge also depends on the correspondence theory of truth and that this too has been seriously challenged by disquotational accounts of truth [Mad 96b, 63-64].

${ }^{20} \mathrm{Cf}$. footnote 19 .
} 
improvement on the original. In fact, I take Field's formulation (or something like it) to be the only formulation of Benacerraf's problem worthy of serious consideration nowdays. How might an indispensabilist respond to this challenge?

The short answer is that the indispensability argument tells us that we come by mathematical knowledge in exactly the same way as other forms of knowledge - by the hypothetico-deductive methods of science [Qui 69a]. How is it that this method is a reliable process? That's a good question, but it's not one that the mathematical realist need be embarrassed by. This is a question that Platonists and nominalists alike must answer. The point of the original Benacerraf challenge and also Field's reformulation is to create a problem for Platonism. To ask after the reliability of the hypothetico-deductive method does not serve this purpose-it's either a problem for everyone or a problem for no-one. Field concedes this possibility:

One could argue [...] that if mathematics is indispensable to laws of empirical science, then if the mathematical facts were different, different empirical consequences could be derived from the same laws of (mathematized) physics. So, it could be said, mathematical facts make an empirical difference, and maybe this would enable the application-based platonist to argue that our observations of the empirical consequences of physical law are enough to explain the reliability of our mathematical beliefs. [Fie 89, 28-29]

Notice that this line of response depends on mathematics being contingent. But as I've already stated (in section 6.4) this is the most plausible position for an indispensabilist to take in any case.

Field, however, has some reservations about this line of response to Benacerraf's challenge. In particular, he is concerned that, given the relatively small amount of mathematics required for empirical science, this line of reasoning does not explain the reliability of very many of our mathematical beliefs. ${ }^{21}$ We have seen this problem before in the guise of Maddy's claim that the Quinean indispensability argument leaves too much mathematics unaccounted for ${ }^{22}$ and the answer I will give here is essentially the same as I gave in response to Maddy: the reliability of our mathematical beliefs may be explained by their applicability to other areas of mathematics, so long as there is a chain of applications that leads eventually to empirical evidence. Such is the nature of applicability in the Quinean holistic view of science. While I

\footnotetext{
${ }^{21} \mathrm{He}$ also comments that the partial success of his nominalisation program exacerbates the situation by reducing the amount of mathematics required by empirical science [Fie 89, 29].

${ }^{22}$ See section 5.1.3, page 94 of this thesis for details.
} 
doubt that this response will entirely satisfy Field, it certainly weakens the point he is making. At the very least, I believe that an appeal to applications in order to explain the reliability of our mathematical beliefs seems a promising avenue to investigate in reply to the Field formulation of the Benacerraf challenge.

Perhaps you are unsatisfied with the whole "short answer" approach to the Benacerraf challenge. Perhaps you'd like to hear more about the nature of the process that connects the individual abstract entities and our knowledge of them. Quine is unwilling to say any more than what I've said above. I too am inclined to think that questions about the process connecting the abstract objects and knowledge of them are misguided. Such questions yearn for causal answers and, as we have already seen, causal accounts of knowledge are generally considered untenable.

Gideon Rosen also subscribes to the view that such questions are misguided [Ros 92, chap 3]. ${ }^{23}$ He points out that the Benacerraf challenge asks for justification of our mathematical beliefs one at a time. According to the indispensability argument, though, mathematical beliefs (indeed, all beliefs) are justified holistically. Someone who subscribes to this argument believes in mathematical entities because of the role they play in our total theory. Such a person should feel no compulsion to justify mathematical beliefs individually, and any demand that he or she do so is entirely unreasonable. ${ }^{24}$

The question of whether Quinean indispensabilists have a satisfactory answer to Benacerraf's challenge is a difficult and complicated matter. Certainly it's far too involved for me to resolve satisfactorily here. Nevertheless, I hope that I've shown that the Quinean has an answer to the challenge. (For what it's worth, I think that it's a good answer, but I don't expect that I've said enough here to convince the disbelievers.) Anyway, I won't pursue this issue further. Instead, I'd like to close with a few remarks about the use of indispensability arguments outside the philosophy of mathematics. In particular, I'd like to say a little about the use of

\footnotetext{
${ }^{23}$ It is difficult to track down explicit formulations of the following argument. I learnt it from Linsky and Zalta [Lin 95, 528] who state the argument without endorsement and they erroneously attributed the argument to the Burgess paper [Bur 90]. The argument may be found implicitly in [Bur 97, 41-49] as well as in much of Quine's work. The only explicit formulations, with endorsement, I'm aware of are in Rosen's PhD thesis (cited above) and in an unpublished manuscript by Smart [Sma 9?]. (My thanks to Bernie Linsky, Ed Zalta, and Gideon Rosen for their help with the detective work involved!)

${ }^{24}$ Some indispensabilists, however, may choose to say more about mathematical epistemology. What is said will obviously depend on the details of what he or she believes mathematical entities to be. For example, someone like the Maddy of Realism in Mathematics [Mad 90] can give a fully causal account of mathematical knowledge since, according to this view, we have causal contact with mathematical objects such as sets. John Bigelow [Big 88] also provides a causal story.
} 
indispensability arguments to motivate belief in concrete possible worlds. Although such arguments have no direct bearing on this thesis, they are worth a mention, since some philosophers feel a slippery slope, leading to modal realism, beckoning when they consider mathematical indispensability arguments.

\subsection{A Slippery Slope?}

David Lewis is clearly a believer in indispensability arguments, arguing, as he does, for concrete, causally isolated possible worlds on the basis of the usefulness of the possible worlds hypothesis:

Why believe in a plurality of worlds?-Because the hypothesis is serviceable, and that is a reason to think that it is true. The familiar analysis of necessity as truth at all possible worlds was only the beginning. In the last two decades, philosophers have offered a great many more analyses that make reference to possible worlds, or to possible individuals that inhabit possible worlds. I find that record most impressive. I think it is clear that talk of possibilia has clarified questions in many parts of the philosophy of logic, of mind, of language, and of science - not to mention metaphysics itself. [Lew 86b, 3]

Even disregarding Lewis's claims about the utility of possible worlds talk for philosophical and logical purposes, the use of such talk in science is perhaps enough to justify the acceptance of possible worlds by a Quinean indispensabilist (who, after all, accepts the purposes of science as legitimate purposes for an indispensability argument). ${ }^{25}$ This places the Quinean indispensabilist in an awkward situation if modal realism is considered unpalatable. (Indeed, I've heard it said that this outcome is a reductio ad absurdum of indispensability theory.)

I have two things to say in response to this concern. Firstly, the indispensabilist's hands are not tied here. Ockhamist concerns might be invoked to put weight behind some of the alternative proposals for dealing with possibilia (such as modal fictionalism). Secondly, possible worlds do not find themselves in the same demand as mathematical entities. After all, most science gets by quite nicely without possible worlds, whereas the same is certainly not true of mathematical entities. From this

\footnotetext{
${ }^{25}$ The scientific use of possible worlds talk is many and varied. Phase planes are best described as spaces of possible initial conditions (and thus, arguably, implicitly invoke possible worlds). Possible worlds have been explicitly invoked to account for certain problems in quantum mechanics concerning the collapse of the wave function and also in cosmology to account for the so-called fine-tuning problem [Cam 94, 37-38].
} 
it might be argued that possible worlds are not in fact indispensable. At the very least, the prospect of denying the indispensability of possible worlds talk on such grounds seems to be one avenue worth exploring.

To be quite honest, I have no firm opinion on this matter. I grant that possible worlds talk is very useful and perhaps even indispensable. On the other hand, such talk is counterintuitive and leads to a very large ontology. One thing is clear: indispensability theory does not rule out modal realism a priori. Some see this itself as a problem, but I take it to be one of the most attractive features of indispensability theory. In fact, I'm highly suspicious of any methodological principle that renders the existence of possible worlds (or whatever) impossible and decides against them on purely a priori grounds. There is nothing inconsistent about possible worlds, and so, at the very least, we must be able to entertain their existence. Indispensability theory allows this. Whether such entities make it all the way into our ontology is another matter. But I hope it is clear by now that the Quine/Putnam indispensability argument does not purport to give easy answers to difficult questions; nor does it rule against interesting and fruitful theses without a fair trial. Surely this is as it should be. 


\section{Appendix A}

\section{Summary}

\section{Chapter 1: Mathematics and its Applications}

In this introductory chapter I gave a general survey of scientific realism and discussed how mathematical realism fitted into the picture. I then discussed indispensability arguments in general terms, before concentrating on the Quine/Putnam argument and how it's used to deliver mathematical realism. I showed how this argument relies on the doctrines of naturalism and holism, so that a defence of both these doctrines is required in a defence of the argument. I also looked at some of the other mathematical indispensability arguments in the literature.

\section{Chapter 2: The Quinean Backdrop}

In this chapter I defended naturalism in the Quinean sense: that is, that philosophy is continuous with science and that we ought to give ontological commitment to the entities of our best scientific theories. I disentangled this position from another influential doctrine that also commonly goes by the name of 'naturalism', and identified the salient difference as rejection or acceptance (respectively) of a causal test called the Eleatic Principle. I then discussed holism, with particular emphasis on what sort of holism indispensability theory requires. Finally, I discussed how Quinean naturalism and confirmational holism combine to yield the crucial first premise of the Quine/Putnam indispensability argument.

\section{Chapter 3: The Eleatic Principle}

In order to defend Quinean naturalism over the causal version it was necessary to undermine the motivation for the Eleatic Principle (or causal criterion) which is the central thesis of the latter. In this chapter I considered some of the most commonly 
appealed to justifications for the Eleatic Principle and in each case I found them wanting. There were two points to be gleaned from the problems with the Eleatic Principle. The first was that these problems served to undermine confidence in the causal version of naturalism thus raising the stocks in its main rival-Quinean naturalism. The second point was that in considering the problems with the Eleatic Principle and how they might be rectified, we found that one is drawn, in a very natural way, to Quinean naturalism. This again highlighted the plausibility of the Quinean position.

\section{Chapter 4: Field's Fictionalism}

In this chapter I considered the influential work of Hartry Field who proposes a bold attack on the indispensability argument by arguing that mathematics is in fact dispensable to science. After a brief outline of Field's program, I set about evaluating the prospects for its success. In order to do this it was necessary to re-examine Quine's indispensability argument, with particular emphasis on what is meant by 'indispensable'. I showed that confirmation theory plays a crucial role in answering this question and that once indispensability is understood in this light, the task of demonstrating the dispensability of mathematics is a much larger task than is often appreciated by many of Field's supporters. This of course didn't counter the Field attack, but it does seem that even in view of his impressive efforts in rewriting much of Newtonian gravitational theory without reference to mathematical objects, it is still an open question whether mathematics is genuinely dispensable to this theory.

\section{Chapter 5: Maddy's Objections}

Penelope Maddy has three objections to the indispensability argument. In this chapter I considered these objections and found that in the cases of the objection from scientific fictions and the objection from the role of mathematics in scientific theories, much of the force of the objections derived from taking the "philosophy must give" reading of naturalism. This, I argued, was a mistake since neither Quine nor Maddy construes naturalism in this way. On the standard reading of Quinean naturalism, the force of these objections is much reduced, and in both cases more than one solution is possible.

The third of Maddy's objections, the mathematical practice objection, was seen to turn on a misconception about Quinean holism. I argued that because a theory is confirmed or disconfirmed as a whole unit does not imply that each fragment of that 
theory has the same priority, as Maddy seems to suggest. When modification of a theory is required, Quine's Maxim of Minimum Mutilation implores us to modify those areas of the overall theory upon which the least depends. The upshot of this was that, given this understanding of holism, once again the Maddy objection could not be sustained.

\section{Chapter 6: Further Objections}

It is well know that any version of mathematical realism motivated by Quinean indispensability arguments must yield a mathematics that is a posteriori and fallible. Such a consequence is considered unacceptable by many, mathematical realists and anti-realists alike. For example, Musgrave and Sober find such a conclusion sits rather uneasily with scientific practice, while Hale and Wright take issue with any account of mathematics that yields the contingent existence of mathematical objects. In this chapter I addressed these and other objections and in doing so I defended the view that mathematical knowledge has an empirical character.

\section{Chapter 7: Conclusion}

In this brief concluding chapter I gave some indication of the varieties of mathematical realism that are consistent with indispensability theory. In particular, I showed that the indispensability argument is silent on many issues such as whether numbers are sets, and whether mathematical entities are causally active or not. I also considered how such accounts stand up to two Benacerrafian concerns, which are generally taken to be serious objections to mathematical realism. These objections are: (i) the indeterminacy of set theoretic reductions of the natural numbers and (ii) the epistemic argument against Platonism. A number of viable, Quinean options were presented for both Benacerraf's worries.

I concluded with a few remarks about the use of indispensability arguments outside of the philosophy of mathematics. In particular, I discussed the worry that the indispensability argument may be used to legitimate belief in possible worlds. I suggested that although it can be, and has been, put to this purpose, belief in possible worlds does not follow as a matter of course from indispensability theory. 



\section{Appendix B}

\section{Notation}

$\aleph_{0} \quad$ The cardinality of the positive integers.

$\aleph_{1} \quad$ The smallest non-denumerable cardinal.

$\beth_{0} \quad \beth_{0}=\aleph_{0}$.

$\beth_{\omega} \quad \beth_{\omega}=\bigcup_{\alpha \in \omega} \beth_{\alpha}$, where $\beth_{\alpha}=2^{\beth_{\alpha-1}}, \alpha$ is an ordinal and $\beth_{0}=\aleph_{0}$.

C The set of complex numbers.

$A \times B \quad$ The cartesian product of the sets $A$ and $B$. That is, $\{(x, y) \mid x \in A$ and $y \in B\}$.

$\nabla \times \mathbf{F} \quad$ The curl of the (vector) function $\mathbf{F}: \mathbb{R}^{3} \rightarrow \mathbb{R}^{3}$.

$\nabla \times \mathbf{F}=\left(\frac{\partial}{\partial y} f_{3}-\frac{\partial}{\partial z} f_{2}\right) \mathbf{i}-\left(\frac{\partial}{\partial x} f_{3}-\frac{\partial}{\partial z} f_{1}\right) \mathbf{j}+\left(\frac{\partial}{\partial x} f_{2}-\frac{\partial}{\partial y} f_{1}\right) \mathbf{k}$,

where $\{\mathbf{i}, \mathbf{j}, \mathbf{k}\}$ is the standard basis for $\mathbb{R}^{3}$,

$\mathbf{F}(x, y, z)=f_{1}(x, y, z) \mathbf{i}+f_{2}(x, y, z) \mathbf{j}+f_{3}(x, y, z) \mathbf{k}$ and $f_{i}: \mathbb{R}^{3} \rightarrow \mathbb{R}, i \in\{1,2,3\}$.

$\delta \quad$ The Dirac delta function. $\delta: \mathbb{R} \rightarrow \mathbb{R}$, such that $\delta(x)=0, \quad \forall x \neq 0$, and $\int_{-\infty}^{+\infty} \delta(x) d x=1$

$\emptyset \quad$ The empty set. (Zero I denote by ' 0 '.)

$f: D \rightarrow C$ Functional notation for $f$ maps from its domain $D$ to its co-domain $C$.

$i \quad i=\sqrt{-1}$.

M $\quad$ One solar mass unit: $1 \mathrm{M}_{\odot} \approx 1.989 \times 10^{33} \mathrm{~g}$

$\mathrm{M}_{\oplus} \quad$ The mass of the Earth: $1 \mathrm{M}_{\oplus} \approx 5.977 \times 10^{27} \mathrm{~g}$.

$\omega \quad$ The first infinite ordinal. The ordinal of the natural order of the positive integers.

$\mathbb{R} \quad$ The set of real numbers.

$\mathbb{R}^{+} \quad$ The set of positive real numbers. 
$\Sigma_{2}^{1} \quad \Sigma_{2}^{1}$ sets are part of the projective hierarchy of sets, obtained by repeated operations of projection and complementation on open sets. The $\Sigma_{2}^{1}$ sets, in particular, are obtained from the open sets (denoted $\Sigma_{0}^{1}$ ) by taking complements to obtain the $\Pi_{0}^{1}$ sets, taking projections of these to obtain the $\Sigma_{1}^{1}$ sets, taking complements of these to obtain the $\Pi_{1}^{1}$ sets and, finally, taking the projections of these to obtain the $\Sigma_{2}^{1}$ sets.

$2^{\aleph_{0}} \quad$ The cardinality of the real numbers (i.e. the cardinality of the power set of the positive integers). 


\section{Bibliography}

[Abe 87] Abell, G.O., Morrison, D. and Wolff, C.S. Exploration of the Universe, fifth edition. Saunders College Publishing, Philadelphia, 1987.

[Ahl 66] Ahlfors, L.V. Complex Analysis. McGraw-Hill, New York, 1966.

[Arm 78] Armstrong, D.M. Universals and Scientific Realism. Cambridge University Press, Cambridge, 1978.

[Arm 80] Armstrong, D.M. The Nature of Mind. Harvester Press, Brighton (Sussex), 1980

[Arm 80a] Armstrong, D.M. Naturalism materialism and first philosophy, in The Nature of Mind. Harvester Press, Brighton (Sussex), 1980, [Arm 80], 149-165.

[Arm 89] Armstrong, D.M. A Combinatorial Theory of Possibility. Cambridge University Press, Cambridge, 1989.

[Azz 94] Azzouni, J. Metaphysical Myths, Mathematical Practice: The Ontology and Epistemology of the Exact Sciences. Cambridge University Press, Cambridge, 1994.

[Azz 97] Azzouni, J. Applied mathematics existential commitment and the QuinePutnam indispensability thesis, Philosophia Mathematica (3), forthcoming.

[Bak 9?] Baker, A. Mathematics indispensability and scientific progress, to appear.

[Bal 96] Balaguer, M. A fictionalist account of the indispensable applications of mathematics, Philosophical Studies, Vol. 83, (1996), 291-314. 
[Ben 65] Benacerraf, P. What numbers could not be, reprinted in Benacerraf, P. and Putnam, H. (eds.) Philosophy of Mathematics Selected Readings, second edition. Cambridge University Press, Cambridge, 1983 (first published in 1965), [Ben 83], 272-294.

[Ben 73] Benacerraf, P. Mathematical truth, reprinted in Benacerraf, P. and Putnam, H. (eds.) Philosophy of Mathematics Selected Readings, second edition. Cambridge University Press, Cambridge, 1983 (first published in 1973), [Ben 83], 403-420.

[Ben 83] Benacerraf, P. and Putnam, H. (eds.) Philosophy of Mathematics Selected Readings, second edition. Cambridge University Press, Cambridge, 1983.

[Ben 96] Benacerraf, P. What mathematical truth could not be - I, in Morton, A. and Stich, S.P. (eds.) Benacerraf and his Critics. Blackwell, Oxford, 1996, [Mor 96], 9-59.

[Ber 73] Bernstein, J. Einstein. Fontana/Collins, Glasgow, 1973.

[Big 88] Bigelow, J. The Reality of Numbers: A Physicalist's Philosophy of Mathematics. Clarendon, Oxford, 1988.

[Big 90] Bigelow, J. and Pargetter, R. Science and Necessity. Cambridge University Press, Cambridge, 1990.

[Boy 86] Boyce, W.E. and DiPrima, R.C. Elementary Differential Equations and Boundary Value Problems, fourth edition. John Wiley, New York, 1986.

[Bur 83] Burgess, J. Why I am not a nominalist, Notre Dame Journal of Formal Logic, Vol. 24, No. 1 (January 1983), 93-105.

[Bur 90] Burgess, J. Epistemology and nominalism, in Irvine, A.D. Physicalism in Mathematics. Kluwer, Dordrecht, 1990, [Irv 90], 1-15.

[Bur 97] Burgess, J. and Rosen, G. A Subject with No Object: Strategies for Nominalistic Interpretation of Mathematics. Clarendon, Oxford, 1997.

[Cam 94] Campbell, K. Selective realism in the philosophy of physics, The Monist, 77, (1994), 27-46.

[Can 9?] Cantini, A., Casari, E. and Minari, P. (eds.) Logic in Florence. Forthcoming. 
[Carn 37] Carnap, R. The Logical Syntax of Language. Routledge and Kegan Paul, London, 1937.

[Cart 83] Cartwright, N. How the Laws of Physics Lie. Oxford University Press, New York, 1983.

[Cha 82] Chalmers, A. What is this thing called Science?, second edition. University of Queensland Press, Brisbane, 1982.

[Che 96] Cheyne, C. and Pigden, C. Pythagorean powers or a challenge to Platonism, Australasian Journal of Philosophy, Vol. 74, No. 4 (December 1996), 639-645.

[Che 98] Cheyne, C. Existence claims and causality, Australasian Journal of Philosophy, forthcoming.

[Chi 73] Chihara, C.S. Ontology and the Vicious-Circle Principle. Cornell University Press, Ithaca (New York), 1973.

[Chi 90] Chihara, C.S. Constructibility and Mathematical Existence. Clarendon, Oxford, 1990.

[Col 97] Colyvan, M. Review of Morton, A. and Stich, S.P. (eds.) Benacerraf and his Critics, Australasian Journal of Philosophy, Vol. 75, No. 2, (June 1997), 262-265.

[Col 98a] Colyvan, M. In defence of indispensability, Philosophia Mathematica (3), Vol. 6, No. 1 (March 1998), forthcoming.

[Col 98b] Colyvan, M. Is Platonism a bad bet?, Australasian Journal of Philosophy, Vol. 76, No. 1 (March 1998), forthcoming.

[Col 9?] Colyvan, M. Confirmation theory and indispensability, Philosophical Studies, forthcoming.

[Dan 92] Dancy, J. and Sosa, E. (eds.) A Companion to Epistemology. Blackwell, Oxford, 1992.

[Dev 77] Devlin, K. The Axiom of Constructibility, Lecture Notes in Mathematics, Vol. 617. Springer-Verlag, Berlin, 1977.

[DIn 92] d'Inverno, R. Introducing Einstein's Relativity. Clarendon, Oxford, 1992. 
[Dir 58] Dirac, P.A.M. The Principles of Quantum Mechanics, fourth edition (revised). Clarendon, Oxford, 1958.

[Duh 06] Duhem, P. The Aim and Structure of Physical Theory. Princeton University Press, Princeton, 1954 (first published in 1906).

[Dum 75] Dummett, M. The philosophical basis of intuitionistic logic, reprinted in Benacerraf, P. and Putnam, H. (eds.) Philosophy of Mathematics Selected Readings, second edition. Cambridge University Press, Cambridge, 1983 (first published in 1975), [Ben 83], 97-129.

[Dum 78] Dummett, M. Truth and Other Enigmas. Duckworth, London, 1978.

[Dum 78a] Dummett, M. Realism, in Truth and Other Enigmas. Duckworth, London, 1978, [Dum 78], 145-165.

[Dum 91] Dummett, M. Frege Philosophy of Mathematics. Duckworth, London, 1991.

[Dys 64] Dyson, F.J. Mathematics in the physical sciences, Scientific American, Vol. 211, No. 3 (Sept 1964), 128-146.

[Ein 05] Einstein, A. On the electrodynamics of moving bodies, reprinted in Einstein, A., Lorentz, H.A., Weyl, H., and Minkowski, H. The Principle of Relativity. Dover, New York, 1923 (first published in 1905), [Ein 23], 35-65.

[Ein 23] Einstein, A., Lorentz, H.A., Weyl, H., and Minkowski, H. The Principle of Relativity. Dover, New York, 1923.

[Ein 67] Einstein, A. The Meaning of Relativity, sixth edition. Chapman and Hall, London, 1967.

[Ell 90] Ellis, B. Truth and Objectivity. Blackwell, Oxford, 1990.

[End 77] Enderton, H.B. Elements of Set Theory. Academic Press, New York, 1977.

[Fey 63] Feynman, R., Leighton, R. and Sands, M. The Feynman Lectures on Physics. Addison-Wesley, Reading (Massachusetts), 1963.

[Fey 65] Feynman, R. The Character of Physical Law. BBC, London, 1965. 
[Fie 80] Field, H. Science Without Numbers: A Defence of Nominalism. Blackwell, Oxford, 1980.

[Fie 84] Field, H. Can we dispense with space-time?, reprinted in Field, H. Realism, Mathematics and Modality. Blackwell, Oxford, 1989 (first published in 1984), [Fie 89], 171-226.

[Fie 85] Field, H. On conservativeness and incompleteness, reprinted in Field, H. Realism, Mathematics and Modality. Blackwell, Oxford, 1989 (first published in 1985), [Fie 89], 125-146.

[Fie 88] Field, H. Realism mathematics and modality, reprinted in Field, H. Realism, Mathematics and Modality. Blackwell, Oxford, 1989 (first published in 1988), [Fie 89], 227-281.

[Fie 89] Field, H. Realism, Mathematics and Modality. Blackwell, Oxford, 1989.

[Fie 92] Field, H. A nominalistic proof of the conservativeness of set theory, Journal of Philosophical Logic, Vol. 21, No. 2 (May 1992), 111-123.

[Fie 93] Field, H. The conceptual contingency of mathematical objects, Mind, Vol. 102, No. 406 (April 1993), 285-299.

[Fod 92] Fodor, J. and Lepore, E. Holism: A Shopper's Guide. Blackwell, Cambridge, 1992.

[For 87] Forrest, P. and Armstrong, D.M. The nature of number, Philosophical Papers, Vol. 16, (November 1987), 165-186.

[For 95] Forrest, P. Is space-time discrete or continuous? - an empirical question, Synthese, Vol. 103, No. 3 (March 1995), 327-354.

[Fre 84] Frege, G. (translated by Austin, J.L.) The Foundations of Arithmetic: A Logico-mathematical enquiry into the concept of number. Blackwell, Oxford, 1950 (first published in 1884).

[Fre 70] Frege, G. (edited by Geach, P. and Black, M.) Translations from the Philosophical Writings of Gottlob Frege. Blackwell, Cambridge, 1970.

[Fri 86] Fricke, M. (ed.) Essays in Honour of Bob Durrant. Otago University Philosophy Department, Dunedin, 1986. 
[Gly 80] Glymour, C. Theory and Evidence. Princeton University Press, Princeton, 1980.

[Göd 47] Gödel, K. What is Cantor's continuum problem?, reprinted (revised and expanded) in Benacerraf, P. and Putnam, H. (eds.) Philosophy of Mathematics Selected Readings, second edition. Cambridge University Press, Cambridge, 1983 (first published in 1947), [Ben 83], 470-485.

[Gri 56] Grice, H.P. and Strawson, P.F. In defence of a dogma, Philosophical Review, LXV, (1956), 141-158.

[Hac 83] Hacking, I. Representing and Intervening. Cambridge University Press, Cambridge, 1983.

[Hah 86] Hahn, L. and Schilpp, P. (eds.) The Philosophy of W.V. Quine. Open Court, La Salle (Illinois), 1986.

[Hal 87] Hale, B. Abstract Objects. Blackwell, Oxford, 1987.

[Hal 92] Hale, B. and Wright, C. Nominalism and the contingency of abstract objects, Journal of Philosophy, Vol. 89, No. 3 (March 1992) 111-135.

[Hal 94] Hale, B. and Wright, C. A reductio ad surdum? Field on the contingency of mathematical objects, Mind, Vol. 103, No. 410 (April 1994) 169-184.

[Ham 80] Hamming, R.W. The unreasonable effectiveness of mathematics, American Mathematics Monthly No. 87 (1980), 81-90.

[Hard 40] Hardy, G.H. A Mathematician's Apology. Cambridge University Press, Cambridge, 1967 (first published in 1940).

[Hart 77] Hart, W.D. Review of Steiner's Mathematical Knowledge, Journal of Philosophy, Vol. 74 (1977), 118-129.

[Hart 96] Hart, W.D. (ed.) The Philosophy of Mathematics. Oxford University Press, Oxford, 1996.

[Hel 89] Hellman, G. Mathematics without Numbers. Clarendon, Oxford, 1989.

[Hel 92] Hellman, G. The boxer and his fists: the constructivist in the arena of quantum physics, Proceedings of the Aristotelian Society, Supplement, LXVI, (1992), 61-77. 
[Hel 9?] Hellman, G. Some ins and outs of indispensability: a modal-structural perspective, in Cantini, A., Casari, E. and Minari, P. (eds.) Logic in Florence. Forthcoming.

[Hem 65] Hempel, C.G. Aspects of Scientific Explanation and Other Essays in the Philosophy of Science. Macmillan, London, 1965.

[Hey 31] Heyting, A. The intuitionistic foundations of mathematics, reprinted in Benacerraf, P. and Putnam, H. (eds.) Philosophy of Mathematics Selected Readings, second edition. Cambridge University Press, Cambridge, 1983 (first published in 1931), [Ben 83], 52-61.

[Hil 99] Hilbert, D. Foundations of Geometry. Open Court, La Salle (Illinois), 1971 (first published in 1899).

[Hor 82] Horwich, P. Probability and Evidence. Cambridge University Press, Cambridge, 1982.

[Irv 90] Irvine, A.D. Physicalism in Mathematics. Kluwer, Dordrecht, 1990.

[Jac 90] Jackson, F. and Pettit, P. Program explanation: a general perspective, Analysis, 50, (1990), 107-117.

[Jac 97] Jackson, F. From Metaphysics to Ethics: A Defence of Conceptual Analysis. Oxford University Press, Oxford, forthcoming.

[Kit 81] Kitcher, P. Explanatory unification, Philosophy of Science, 48, (1981), $507-531$.

[Kit 84] Kitcher, P. The Nature of Mathematical Knowledge. Oxford University Press, New York, 1984.

[Kli 72] Kline, M. Mathematical Thought from Ancient to Modern Times. Oxford University Press, New York, 1972.

[Kos 80] Kosniowski, C. A First Course in Algebraic Topology. Cambridge University Press, Cambridge, 1980.

[Kri 80] Kripke, S. Naming and Necessity. Blackwell, Oxford, 1980.

[Lak 70] Lakatos, I. and Musgrave, A. (eds.) Criticism and the Growth of Knowledge, Cambridge University Press, Cambridge, 1970. 
[Lak 70a] Lakatos, I. Falsification and the methodology of scientific research programmes, in Lakatos, I. and Musgrave, A. (eds.) Criticism and the Growth of Knowledge, Cambridge University Press, Cambridge, 1970, [Lak 70], 91-195.

[Lak 76] Lakatos, I. Proofs and Refutations: The Logic of Mathematical Discovery. Cambridge University Press, Cambridge, 1976.

[Lew 86] Lewis, D. Philosophical Papers Vol. II. Oxford University Press, New York, 1986.

[Lew 86a] Lewis, D. Causal explanation, in Philosophical Papers Vol. II. Oxford University Press, New York, 1986, [Lew 86], 214-240.

[Lew 86b] Lewis, D. On the Plurality of Worlds. Blackwell, Oxford, 1986.

[Lew 91] Lewis, D. Parts of Classes. Blackwell, Oxford, 1991.

[Lin 95] Linsky, B. and Zalta, E. Naturalized Platonism versus Platonized naturalism, Journal of Philosophy, Vol. 92, No. 10 (October, 1995), 525-555.

[Lin 9?] Linsky, B. Placing abstract objects in naturalism, unpublished manuscript.

[Lor 04] Lorentz, H.A. Electromagnetic phenomena in a system moving with any velocity less than that of light, reprinted in Einstein, A., Lorentz, H.A., Weyl, H., and Minkowski, H. The Principle of Relativity. Dover, New York, 1923 (first published in 1904), [Ein 23], 9-34.

[Lor 85] Lorentz, H.A. Michelson's interference experiment, reprinted in Einstein, A., Lorentz, H.A., Weyl, H., and Minkowski, H. The Principle of Relativity. Dover, New York, 1923 (first published in 1885), [Ein 23], 1-7.

[Mad 90] Maddy, P. Realism in Mathematics. Clarendon, Oxford, 1990.

[Mad 92] Maddy, P. Indispensability and practice, Journal of Philosophy, Vol. 89, No. 6 (June 1992), 275-289.

[Mad 94] Maddy, P. Taking naturalism seriously, in Prawitz, D., Skyrms, B. and Westerståhl, D. (eds.) Logic, Methodology and Philosophy of Science IX. Elsevier, Amsterdam, 1994, 383-407. 
[Mad 95a] Maddy, P. Naturalism and ontology, Philosophia Mathematica (3), Vol. 3, No. 3 (September 1995), 248-270.

[Mad 95b] Maddy, P. How to be a naturalist about mathematics, paper delivered to the 'Truth in Mathematics' Conference in Mussomeli, Italy, September 1995.

[Mad 96a] Maddy, P. Set theoretic naturalism, The Journal of Symbolic Logic, Vol. 61, No. 2 (June 1996), 490-514.

[Mad 96b] Maddy, P. The legacy of 'Mathematical Truth', in Morton, A. and Stich, S.P. (eds.) Benacerraf and his Critics. Blackwell, Oxford, 1996, [Mor 96], 60-72.

[Mad 97] Maddy, P. Naturalism in Mathematics. Clarendon, Oxford, forthcoming.

[Mad 97a] Maddy, P. Naturalizing mathematical methodology, in Schirn, M. (ed.) Philosophy of Mathematics Today. Clarendon, Oxford, forthcoming, [Sch 97].

[Mad 9?] Maddy, P. Ontological commitment: between Quine and Duhem, Philosophical Perspectives, forthcoming.

[Mal 82] Malament, D. Review of Field's Science Without Numbers, Journal of Philosophy, Vol. 79, (1982), 523-534.

[Mar 95] Martin, D.A. Mathematical evidence, paper delivered to the "Truth in Mathematics' Conference in Mussomeli, Italy, September 1995.

[McC 94] McCleary, J. Geometry from a Differentiable Viewpoint. Cambridge University Press, Cambridge, 1994.

[Mil 43] Mill, J.S. A System of Logic, New impression. Longmans, Green and Co., London, 1947 (first published in 1843).

[Mor 96] Morton, A. and Stich, S.P. (eds.) Benacerraf and his Critics. Blackwell, Oxford, 1996

[Mus 86] Musgrave, A. Arithmetical Platonism: Is Wright wrong or must Field yield?, in Fricke, M. (ed.) Essays in Honour of Bob Durrant. Otago University Philosophy Department, Dunedin, 1986, [Fri 86], 90-110. 
[Odd 82] Oddie, G. Armstrong on the Eleatic principle and abstract entities, Philosophical Studies 41, (1982), 285-295.

[Pap 93] Papineau, D. Philosophical Naturalism. Blackwell, Oxford, 1993.

[Par 80] Parsons, C. Mathematical intuition, reprinted in Hart, W.D. (ed.) The Philosophy of Mathematics. Oxford University Press, Oxford, 1996 (first published in 1980), [Hart 96], 95-113.

[Par 83] Parsons, C. Mathematics in Philosophy, Cornell University Press, Ithaca (New York), 1983.

[Par 83a] Parsons, C. Quine on the philosophy of mathematics, in Mathematics in Philosophy. Cornell University Press, Ithaca (New York), 1983, [Par 83], 176-205.

[Pea 92] Peat, F.D. Superstrings and the search for the theory of everything. Abacus, London, 1992.

[Pla 35] Plato, Sophist, trans. Cornford, F.M. in Plato's Theory of Knowledge. Kegan Paul, London, 1935.

[Pric 96] Price, H. Time's Arrow and Archimedes' Point: New Directions for the Physics of Time. Oxford University Press, Oxford, 1996.

[Pric 98] Price, H. The role of history in microphysics, forthcoming in Sankey, H. (ed.) Causation and Laws of Nature. Kluwer, Dordrecht, forthcoming, [San 98].

[Prie 79] Priest, G. Two dogmas of Quineanism, Philosophical Quarterly, Vol. 29, No. 117, (October 1979), 289-301.

[Prie 9?] Priest, G. Finite models of arithmetic, Journal of Philosophical Logic, forthcoming.

[Put 67] Putnam, H. Mathematics without foundations, reprinted in Mathematics Matter and Method: Philosophical Papers Vol. 1, second edition. Cambridge University Press, Cambridge, 1979 (first published in 1967), [Put 79], 43-59. 
[Put 71] Putnam, H. Philosophy of logic, reprinted in Mathematics Matter and Method: Philosophical Papers Vol. I, second edition. Cambridge University Press, Cambridge, 1979 (first published in 1971), [Put 79], 323-357.

[Put 73] Putnam, H. Reference and meaning, Journal of Philosophy 70, (1973), 699-711.

[Put 76] Putnam, H. Two dogmas revisited, reprinted in Realism and Reason: Philosophical Papers Vol. 3. Cambridge University Press, Cambridge, 1983 (first published in 1976), [Put 83], 87-97.

[Put 79] Putnam, H. Mathematics Matter and Method: Philosophical Papers Vol. I, second edition. Cambridge University Press, Cambridge, 1979.

[Put 79a] Putnam, H. What is mathematical truth?, in Mathematics Matter and Method: Philosophical Papers Vol. 1, second edition. Cambridge University Press, Cambridge, 1979, [Put 79], 60-78.

[Put 79b] Putnam, H. Analyticity and apriority: beyond Wittgenstein and Quine, reprinted in Realism and Reason: Philosophical Papers Vol. 3. Cambridge University Press, Cambridge, 1983 (first published in 1979), [Put 83], 115-138.

[Put 83] Putnam, H. Realism and Reason: Philosophical Papers Vol. 3. Cambridge University Press, Cambridge, 1983.

[Qui 36] Quine, W.V. Truth by convention, reprinted in Benacerraf, P. and Putnam, H. (eds.) Philosophy of Mathematics Selected Readings, second edition. Cambridge University Press, Cambridge, 1983 (first published in 1936), [Ben 83], 329-354.

[Qui 48] Quine, W.V. On what there is, reprinted in From a Logical Point of View, second edition. Harvard University Press, Cambridge (Massachusetts), 1980 (first published 1948), [Qui 53], 1-19.

[Qui 51] Quine, W.V. Two dogmas of empiricism, reprinted in From a Logical Point of View, second edition. Harvard University Press, Cambridge (Massachusetts), 1980 (first published in 1951), [Qui 53], 20-46.

[Qui 53] Quine, W.V. From a Logical Point of View, second edition. Harvard University Press, Cambridge (Massachusetts), 1961 (first edition 1953). 
[Qui 53a] Quine, W.V. On mental entities, reprinted in The Ways of Paradox and Other Essays, revised edition. Harvard University Press, Cambridge (Massachusetts), 1976 (first published in 1953), [Qui 76], 221-227.

[Qui 60] Quine, W.V. Word and Object. Massachusetts Institute of Technology Press and John Wiley and Sons, New York, 1960.

[Qui 60a] Quine, W.V. Posits and reality, reprinted in The Ways of Paradox and Other Essays, revised edition. Harvard University Press, Cambridge (Massachusetts), 1976 (first published in 1960), [Qui 76], 246-254.

[Qui 63] Quine, W.V. Carnap and logical truth, reprinted in Benacerraf, P. and Putnam, H. (eds.) Philosophy of Mathematics Selected Readings, second edition. Cambridge University Press, Cambridge, 1983 (first published in 1963), [Ben 83], 355-376.

[Qui 69] Quine, W.V. Ontological Relativity and Other Essays. Columbia University Press, New York, 1969.

[Qui 69a] Quine, W.V. Epistemology naturalised, in Ontological Relativity and Other Essays. Columbia University Press, New York, 1969, [Qui 69], 69-90.

[Qui 69b] Quine, W.V. Existence and quantification, in Ontological Relativity and Other Essays. Columbia University Press, New York, 1969, [Qui 69], 91113.

[Qui 74] Quine, W.V. The Roots of Reference. Open Court, La Salle (Illinois), 1974.

[Qui 76] Quine, W.V. The Ways of Paradox and Other Essays, revised edition. Harvard University Press, Cambridge (Massachusetts), 1976.

[Qui 81] Quine, W.V. Theories and Things. Harvard University Press, Cambridge (Massachusetts), 1981.

[Qui 81a] Quine, W.V. Five milestones of empiricism, in Theories and Things. Harvard University Press, Cambridge (Massachusetts), 1981, [Qui 81], $67-72$. 
[Qui 81b] Quine, W.V. Success and limits of mathematization, in Theories and Things. Harvard University Press, Cambridge (Massachusetts), [Qui 81], $1981,148-155$.

[Qui 86] Quine, W.V. Reply to Charles Parsons, in Hahn, L. and Schilpp, P. (eds.) The Philosophy of W.V. Quine. Open Court, La Salle (Illinois), 1986, [Hah 86], 396-403.

[Qui 90] Quine, W.V. Pursuit of Truth, revised edition. Harvard University Press, Cambridge (Massachusetts), 1992 (first edition 1990).

[Qui 95] Quine, W.V. From Stimulus to Science. Harvard University Press, Cambridge (Massachusetts), 1995.

[Res 81] Resnik, M. Mathematics as a science of patterns: ontology and reference, Noûs, Vol. 15, No. 4 (November 1981), 529-550.

[Res 82] Resnik, M. Mathematics as a science of patterns: epistemology, Noûs, Vol. 16, No. 1 (March 1982), 95-105.

[Res 83] Resnik, M. Review of Hartry Field's Science Without Numbers, Noûs, Vol. 17 (1983), 514-519.

[Res 85a] Resnik, M. How nominalist is Hartry Field's nominalism?, Philosophical Studies, No. 47 (1985), 163-181.

[Res 85b] Resnik, M. Ontology and logic: remarks on Hartry Field's anti-platonist philosophy of mathematics, History and Philosophy of Logic, No. 6 (1985), 191-209.

[Res 95] Resnik, M. Scientific vs. mathematical realism: the indispensability argument, Philosophia Mathematica (3), Vol. 3, No. 2 (May 1995), 166-174.

[Res 97] Resnik, M. Mathematics as a Science of Patterns. Clarendon, Oxford, 1997.

[Res 97a] Resnik, M. Holistic mathematics, in Schirn, M. (ed.) Philosophy of Mathematics Today. Clarendon, Oxford, forthcoming, [Sch 97].

[Ris 94] Riskin, A. On the most open question in the history of mathematics: a discussion of Maddy, Philosophia Mathematica (3), Vol. 2, No. 2 (May 1994), 109-121. 
[Ros 92] Rosen, G. 'Remarks on modern nominalism', PhD dissertation, Princeton, 1992.

[Rus 20] Russell, B. Introduction to Mathematical Philosophy. Dover, New York, 1993 (first published in 1920).

[Rus 24] Russell, B. Philosophy in the twentieth century, reprinted in Sceptical Essays, Sixth impression. George Allen and Unwin, London, 1956 (first published in 1924), [Rus 56], 54-79.

[Rus 56] Russell, B. Sceptical Essays, Sixth impression. George Allen and Unwin, London, 1956.

[San 98] Sankey, H. Causation and Laws of Nature, Kluwer, Dordrecht, forthcoming.

[Sch 97] Schirn, M. (ed.) Philosophy of Mathematics Today. Clarendon, Oxford, forthcoming.

[Sho 83] Shope, R. The Analysis of Knowing: A Decade of Research. Princeton University Press, Princeton, 1983.

[Sin 97] Singh, S. Fermat's Last Theorem: The Story of a Riddle that Confounded the World's Greatest Minds for 358 Years. Fourth Estate, London, 1997.

[Sma 63] Smart, J.J.C. Philosophy and Scientific Realism. Routledge and Kegan Paul, London, 1963.

[Sma 90] Smart, J.J.C. Explanation-opening address, Knowles, D. (ed.) Explanation and its Limits. Cambridge University Press, Cambridge, 1990, $1-19$.

[Sma 9?] Smart, J.J.C. Prospects for the philosophy of mathematics, unpublished manuscript.

[Sob 93] Sober, E. Mathematics and indispensability, Philosophical Review, Vol. 102, No. 1 (January 1993), 35-57.

[Ste 73] Steiner, M. Platonism and the causal theory of knowledge, Journal of Philosophy Vol. 70 (1973), 57-66. 
[Ste 75] Steiner, M. Mathematical Knowledge. Cornell University Press, Ithaca (New York), 1975.

[Ste 89] Steiner, M. The application of mathematics to natural science, Journal of Philosophy, Vol. 86, No. 9 (September 1989), 449-480.

[Ste 95] Steiner, M. The applicabilities of mathematics, Philosophia Mathematica (3), Vol. 3, No. 2 (May 1995), 129-156.

[vanF 80] van Fraassen, B. The Scientific Image. Clarendon, Oxford, 1980.

[Wag 96] Wagner, S.J. Prospects for Platonism, in Morton, A. and Stich, S.P. (eds.) Benacerraf and his Critics. Blackwell, Oxford, 1996, [Mor 96], 7399.

[Wei 93] Weinberg, S. Dreams of a Final Theory. Vintage, London, 1993.

[Wig 60] Wigner, E.P. The unreasonable effectiveness of mathematics in the natural sciences, Communications on Pure and Applied Mathematics, Vol. 13 (Feb 1960), 1-14.

[Wil 44] Williams, D.C. Naturalism and the nature of things, reprinted in Principles of Empirical Realism. Charles Thomas, Springfield (Illinois), 1966 (first published 1944), 212-238.

[Wil 66] Williams, D.C. Principles of Empirical Realism. Charles Thomas, Springfield (Illinois), 1966.

[Wol 92] Wolszczan, A. and Frail, D.A. A planetary system around the millisecond pulsar PSR1257+12, Nature, Vol 355 (9 January 1992), 145-147.

[Wri 83] Wright, C. Frege's Conception of Numbers as Objects. Aberdeen University Press, Aberdeen, 1983.

[Wri 88] Wright, C. Why numbers can believably be, Revue Internationale de Philosophie, Vol 42 (1988), 425-473.

[Wri 92] Wright, C. Truth and Objectivity. Harvard University Press, Cambridge, (Massachusetts), 1992. 



\section{Index}

Abel, 109

Adams, 44

Casullo, 120n

Ahlfors, $79 \mathrm{n}$

Cauchy, 79

Chalmers, 35

Ampère, 81-82

Chandrasekar, 50

Aristotle, 27, 29

Chase, 121n

Armstrong, 39

abstract objects, 22, 22n

Cheyne, 125n, 149

Eleatic Principle, 43-45

indispensability, 145-149

62

naturalism, 22, 25, 32-33

numbers as universals, 144

Arnowitt, 49n

Azzouni, 99n, 108n

Bartnik, 49n

Benacerraf, 19

epistemology, 1, 153-156

indeterminacy, 1, 150-153

parsimony, 144

reductions, $144 \mathrm{n}$

semantics, 15-16

Bigelow, 45n

causal mathematical entities, 4, 156n

numbers as universals, 144

problems with indispensability, 119 122

Boolos, 150

Burgess, 66n, 156n

Campbell, 40, 60n

Cantor, 95

Cardan, 104

Carnap, 37

Cartwright, 8, 39, 56, 58-60

Chihara, 65, 96n

Craig, 75, 87, 129n

Darwin, 27

Descartes, 84n, 87n, 104

Deser, $49 \mathrm{n}$

Devlin, $95 \mathrm{n}$

Dirac, 6, 82-83, 103-104, 104

Dretske, 55

Duhem, 34, 34n, 35, 58

Dummett, 2-3, 9, 150

Dyson, $78 \mathrm{n}$

Eddington, 125

Einstein, 27, 47, 49, 53, 76, 84, 85n, 110 thought experiments, $30-31$

Ellis, 39, 42, 56, 56-58

Euclid, 126

Euler, 104

Faraday, 81

Fermat, 2, 2n

Feynman, 82n, 96n, 103

Field, 4, 39, 42, 89n, 118

conservativeness, 68, 68-70, 123, 125

consistency, 69, 137-138 
contingency, 116, 137-142

entailment, 69

epistemology, 154-156

fictionalism, 5, 65-66, 117

indispensability, $1,6,18,65-66,94 \mathrm{n}$, 143

insularity, 141

motivation for nominalism, 67

nominalisation, $68,70-73,122 \mathrm{n}, 125$, 145,148

parsimony, 151

Platonistic methods, 68

representation theorem, $72-73$

virtues of nominalism, $86-88$

FitzGerald, 52-54, 84, 85

Fodor, 34

Forrest, 45n, 136n, 144

Foucault, 124

Frail, 44

Frege, 13, 15n, 115, 118, 130, 150, 151 indispensability, $8-9$

Gödel, 3, 13, 95, 122

indispensability, 9

Galileo, 29-30

Galle, 44

Gauss, 47, 81, 105

Gettier, 153n

Glymour, 76n

Gold, 113n

Gosse, 128, 131

Hacking, 40n, 58

Hale, 15n, 116, 137-142

Hardy, 105

Hart, 154

Heisenberg, 109

Hellman, 131-137 modal structuralism, 144

Hempel, 76n

Hertz, 82
Hilbert, 71-72, 87, 87n

Horwich, $76 \mathrm{n}$

Jackson, 29, 31, 54-55, 118n

Kant, 28, 120

Kepler, 27

Khlentzos, 113n

Kitcher, 47n, 76, 140

Kripke, 137n

Kuhn, 101

Lakatos, 35-36

Lebesgue, 95, 109

Lepore, 34

Leverrier, 44

Lewis

mereology, 144

modal realism, 41n, 157-158

non-causal explanation, $47 \mathrm{n}, 50-51$

rejecting mathematics, 33

Lie, 6

Linsky, 15n, 17n, 22n, 131n, 156n

Lorentz, 52-54, 83-85

Maddy, 3, 113n

epistemology, 154, 154n

indispensability, 94-95

mathematical fictions, 93, 102-105

mathematical practice, 93-96, 105111

naturalism, 96-99, 107

problems with indispensability, 18,65 ,

$88,91-113,143$

scientific fictions, 92, 99-101

set theoretic realism, 4, 5, 156n

$V=L, 95-96,112-113$

Maxwell, 81-82, 84, 85

Meinong, $15 \mathrm{n}$

Mendeleeff, 45, 60

Menzies, $89 \mathrm{n}$

Michelson, 76, 84 
Mill, 115

Minkowski, 49, 52-54

Misner, 49n

Moore, 14

Morley, 76, 84

Musgrave, 61n, 116, 123-127

Newton, 27, 77, 81, 84, 104, 110, 124, 126 , 130-132

Oddie, $32,40,46$

Oppy, 89 n

Ostwald, 92, 101

Papineau, 22, 79

Pargetter, 119n

Parsons, 116-119, 126

Pauli, 50, 50, 51n, 83

Peano, 121, 122, 138

Pettit, $54-55$

Pigden, 125n, 145-149

Planck, 136n

Plato, 32

Poincaré, 85n, 92, 100-101

Popper, 123

Priest, 27

Ptolemy, 27, 126

Putnam, 28, 31n goals of science, 11 indispensability, $10-11,13,57 \mathrm{n}$ intellectual dishonesty, 10-11, 100 modal structuralism, 3, 144 realism, 2-3 twin earth, 30

Pythagoras, 27

Quine, 76n, 113n, 118 a priori, $27-28,120 \mathrm{n}$ confirmational holism, 34-37 continuity thesis, 24 desert landscapes, 144 epistemology, $156 \mathrm{n}$ first philosophy, 24

indispensability, 1, 10-13

limit myths, 99n

mathematical recreation, 108

minimum mutilation, 111, 112, 127

modality, $125,137 \mathrm{n}$

naturalism, 12-13, 18, 22, 23-26, 41, 96-99, 107

ontic commitments, $24,39,62$

parsimony, 151

quantification, $74 \mathrm{n}$

semantic holism, 34

sets, 144

unapplied mathematics, 94, 107

$V=L, 95 \mathrm{n}, 112-113$

web of belief, 116

Resnik, 89n, 106n, 113n, 126

indispensability, 14-15, 102n, 129n

patterns, 144

scope of theories, 110-111, 112

structuralism, $152 \mathrm{n}$

Riskin, 109

Roeper, 89n

Rosen, 156, 156n

Russell, 17, 22n, 150, 151

Schrödinger, 83

Schwarzchild, 49n

Smart, 8n, 46n, 89n, 149, 156n

Sober, 65, 116, 121n, 127-137, 143

Spillane, $49 n$

Steiner, 83n, 153n

Tarski, 16

van Fraassen, 8, 24, 58, 127, 128

von Neumann, 1n, 5, 152

Wagner, 15n, 16-17

Weinberg, 76n

Whittaker, $85 \mathrm{n}$ 
Wigner, 6, 86

Williams, 22n

Winckler, 44-45

Wolszcan, 44

Wright, 15n, 116, 137-142, 145

Zalta, 15n, 156n

Zermelo, 1n, 5, 152 